JOURNAL OF THE AMERICAN MATHEMATICAL SOCIETY

Volume 9, Number 4, October 1996

\title{
THETA DICHOTOMY FOR UNITARY GROUPS
}

\author{
MICHAEL HARRIS, STEPHEN S. KUDLA, AND WILLIAM J. SWEET
}

\section{INTRODUCTION}

Some recent work of Gross and Prasad [14] suggests that the root numbers attached to certain symplectic representations of the Weil-Deligne group of a local field $F$ control certain branching rules for representations of orthogonal groups over $F$. On a global level, this local phenomenon should have implications for the structure of the value at the center of symmetry for certain L-functions of arithmetic interest [12]. This conjectural picture is based, to some extent, on the now classic work of Tunnell [47] and Waldspurger [49] as well as on the case of the triple product L-function $[13,34,17]$. In all of these examples, the local root number detects the existence of a certain type of invariant linear functional. It is possible to set up analogous conjectures for the isometry groups of Hermitian or quaternion-hermitian forms.

It turns out that root numbers also play a role in the local and global theta correspondence. Roughly speaking, certain local root numbers should control the occurrence of representations in the local theta correspondence between groups of the 'same size', e.g., for dual pairs of the form $(S p(n), O(2 n+1))$ and $(U(n), U(n))$. As will be seen, the theta correspondence for such pairs is connected with a certain induced representation $I_{n}(s, \chi)$ at the point $s=0$ on the unitary axis. By contrast, the correspondence for the pairs $(S p(n), O(2 n))$ and $(S p(n), O(2 n+2))$, discussed by Prasad [35], is connected to the behavior of a similar induced representation at the points $\pm \frac{1}{2}$, in which case no epsilon factor arises.

In this paper we consider the local theta correspondence for unitary groups in the non-archimedean case. Let $F$ be a non-archimedean local field of characteristic not equal to 2, and let $E$ be a quadratic extension of $F$. For further notation, see the notation section at the end of this introduction. Let $V$ and $W$ be $E$ vector spaces of dimensions $m$ and $n$ equipped, respectively, with a Hermitian form $():, V \times V \longrightarrow E$ and a skew-Hermitian form $\langle\rangle:, W \times W \longrightarrow E$. Then the isometry groups $G(V)$ and $G(W)$ of the spaces $V$ and $W$ form a dual reductive pair in the symplectic group $S p(\mathbb{W})$, where $\mathbb{W}=V \otimes_{E} W$, viewed as an $F$ vector space of dimension $2 m n$ and equipped with the symplectic form

$$
\left\langle\left\langle v_{1} \otimes w_{1}, v_{2} \otimes w_{2}\right\rangle\right\rangle=\frac{1}{2} \operatorname{tr}_{E / F}\left(\left(v_{1}, v_{2}\right) \overline{\left\langle w_{1}, w_{2}\right\rangle}\right) .
$$

Received by the editors May 11, 1994 and, in revised form, April 19, 1995.

1991 Mathematics Subject Classification. Primary 11F27, 11R39, $20 \mathrm{E} 50$.

Partially supported by NSF Grants number: DMS-9203142 (the first author) and DMS-9302539 (the second author). 
For a fixed non-trivial additive character $\psi$ of $F$, let $\omega_{\psi}$ be the smooth Weil representation of the metaplectic cover $M p(\mathbb{W})$ (we view this as an extension of $S p(\mathbb{W}$ ) by $\left.\mathbb{C}^{1}\right)$ realized on a space $S$. For any choice of a pair of characters $\chi=\left(\chi_{1}, \chi_{2}\right)$ of $E^{\times}$whose restrictions to $F^{\times}$satisfy $\left.\chi_{1}\right|_{F^{\times}}=\epsilon_{E / F}^{n}$, and $\left.\chi_{2}\right|_{F^{\times}}=\epsilon_{E / F}^{m}$, there exists a homomorphism

$$
\tilde{\iota}_{\chi}: G(V) \times G(W) \longrightarrow M p(\mathbb{W})
$$

lifting the natural map

$$
\iota: G(V) \times G(W) \longrightarrow S p(\mathbb{W}),
$$

and so, we obtain a representation $\omega_{\chi}$ of $G(V) \times G(W)$ on $S$. For an irreducible admissible representation $\pi$ of $G(W)$, we say that $\pi$ occurs in the local theta correpondence for the dual pair $G(V), G(W)$ (and write $\Theta_{\chi}(\pi, V) \neq 0$ ) if $\pi$ occurs as a quotient of $\omega_{\chi}$. The local Howe duality principle then asserts that there is a uniquely determined irreducible admissible representation $\theta_{\chi}(\pi, V)$ of $G(V)$ such that

$$
\operatorname{Hom}_{G(V) \times G(W)}\left(\omega_{\chi}, \theta_{\chi}(\pi, V) \otimes \pi\right) \neq 0,
$$

and that the representations $\theta_{\chi}\left(\pi_{1}, V\right)$ and $\theta_{\chi}\left(\pi_{2}, V\right)$ corresponding to distinct $\pi$ 's are distinct. This principle is known to hold whenever the residue characteristic of $F$ is not $2[50]$.

For a fixed dimension $m$ there are precisely two isomorphism classes of (nondegenerate) Hermitian spaces over $E$, and these are distinguished by the coset $(-1)^{\frac{m(m-1)}{2}} \operatorname{det} V \cdot N E^{\times}$in $F^{\times} / N E^{\times}$, where $\operatorname{det} V$ denotes the determinant of the matrix $\left(\left(v_{i}, v_{j}\right)\right)$ of inner products for any $E$ basis $\left\{v_{1}, \ldots, v_{m}\right\}$. We denote these spaces by $V_{m}^{ \pm}$, so that

$$
\epsilon_{E / F}\left((-1)^{\frac{m(m-1)}{2}} \operatorname{det} V_{m}^{ \pm}\right)= \pm 1,
$$

and we consider the collection of representations $\theta_{\chi}\left(\pi, V_{m}^{ \pm}\right)$attached to a fixed representation $\pi$ of $G(W)$ as $m$ and \pm vary.

The main dichotomy phenomenon referred to in the title is the following:

Theta dichotomy (Corollary 4.4). Suppose that $\pi$ is a supercuspidal representation of $G(W)$, where $\operatorname{dim}_{E} W=n$. Then, for a fixed character $\chi$ of $E^{\times}$with $\left.\chi\right|_{F^{\times}}=\epsilon_{E / F}^{n}$, precisely one of the representations $\theta_{\chi}\left(\pi, V_{n}^{ \pm}\right)$is non-zero.

Here we are taking $\chi_{1}=\chi_{2}=\chi$, in a slight abuse of the notation above.

Thus, the supercuspidal part of the sum $S_{\chi}\left(V_{n}^{+}\right) \oplus S_{\chi}\left(V_{n}^{-}\right)$of the Weil representations of $U(n)$ attached to the two Hermitian spaces of dimension $n$ provides a 'model' for the supercuspidal representations for $U(n)$, in the sense that one has

$$
\left(S_{\chi}\left(V_{n}^{+}\right) \oplus S_{\chi}\left(V_{n}^{-}\right)\right)_{\text {s.c. }} \simeq \bigoplus_{\pi \text { s.c. }} \pi \otimes \theta_{\chi}\left(\pi, V_{n}^{ \pm}\right) .
$$

Here we take the non-zero space $\theta_{\chi}\left(\pi, V_{n}^{ \pm}\right)$. 
In fact, theta dichotomy should hold for all irreducible admissible representations $\pi$, and we prove this for many non-supercuspidals (Corollary 4.4). For example, we also show that for any $\pi$, and for $m \geq n$, at least one of the representations $\theta_{\chi}\left(\pi, V_{m}^{+}\right)$and $\theta_{\chi}\left(\pi, V_{m}^{-}\right)$is non-zero (Corollary 4.5).

The non-vanishing of $\theta_{\chi}\left(\pi, V_{n}^{ \pm}\right)$is controlled by a local root number which is defined as follows. For an irreducible admissible representation $\pi$ of $G(W)$ and a character $\chi$ of $E^{\times}$, Piatetski-Shapiro and Rallis [31,32,33] defined a family of zeta integrals $\{Z(s, \phi, \Phi)\}$ and obtained from it an L-factor, $L(s, \pi, \chi)=L_{P S R}(s, \pi, \chi)$, and $\epsilon$-factor, $\epsilon(s, \pi, \chi, \psi)=\epsilon_{P S R}(s, \pi, \chi, \psi ; W)$. If the space $W$ is unramified (see the notation section below), if $\chi$ is unramified, and if $\pi$ is an unramified principal series representation, then

$$
L_{P S R}(s, \pi, \chi)=L(s, B C(\pi) \otimes \chi)
$$

and

$$
\epsilon_{P S R}(s, \pi, \chi, \psi ; W)=\epsilon\left(s, B C(\pi) \otimes \chi, \psi_{E}\right),
$$

where the factors on the right are the standard $\mathrm{L}$ and $\epsilon$-factors $[19,11]$ for the base change, [5], $B C(\pi)$ of $\pi$ to $G L_{n}(E)$, twisted by $\chi$. Here we note that the definition of $\epsilon$ given in [32] depends on an auxiliary choice, and the dependence on that choice is not made explicit there. In section 6 , we determine this dependence and give an unambiguous definition of $\epsilon_{P S R}(s, \pi, \chi, \psi ; W)$, which is, of course, crucial for us. This factor actually depends on the isomorphism class of the space $W((0.19)$ and Proposition 6.11).

Epsilon Dichotomy (Theorem 6.1). For a supercuspidal representation $\pi$ of $G(W)$, and for a fixed character $\chi$ of $E^{\times}$with $\left.\chi\right|_{F^{\times}}=\epsilon_{E / F}^{n}, n=\operatorname{dim}_{E} W$,

$$
\theta_{\chi}(\pi, V) \neq 0 \Longleftrightarrow \epsilon_{P S R}\left(\frac{1}{2}, \pi, \chi, \psi ; W\right)=\epsilon_{E / F}(-2)^{n} \cdot \epsilon_{E / F}(\operatorname{det} V) .
$$

Here $V$ is a Hermitian space with $\operatorname{dim}_{E} V=n$.

Again, we expect that this result holds for all irreducible admissible representations of $G(W)$.

This dichotomy phenomenon provides information about the structure of the local theta correspondence for unitary groups. Some of the nicest properties of that local correspondence involve its compatibility with Witt towers. Let $V_{r, r}$ denote the Hermitian space of dimension $2 r$ over $E$ with maximal isotropic subspaces of dimension $r$, so that, with the notation introduced above, $V_{r, r} \simeq V_{2 r}^{+}$. The collections of spaces $\left\{V_{2 r}^{+} \mid r \geq 0\right\}$ and $\left\{V_{2 r}^{-} \mid r \geq 1\right\}$ are then the two Witt towers of even dimensional Hermitian spaces. Similarly, $V_{1}^{ \pm} \simeq E$ is the one dimensional Hermitian space over $E$ with Hermitian form $(x, y)=\alpha \bar{x} y$, where $\alpha \in F^{\times}$with $\epsilon_{E / F}(\alpha)= \pm 1$, and $V_{2 r+1}^{ \pm} \simeq V_{1}^{ \pm}+V_{r, r}$. The collections $\left\{V_{2 r+1}^{+} \mid r \geq 0\right\}$ and $\left\{V_{2 r+1}^{-} \mid r \geq 0\right\}$ are then the two Witt towers of odd dimensional Hermitian spaces. If we fix an element $\delta \in E^{\times}$with $\bar{\delta}=-\delta$, then to every skew-Hermitian space $W$, $\langle$,$\rangle , over E$ we may associate a Hermitian space $W^{\prime}=W$ with $(x, y)^{\prime}=\delta^{-1}\langle y, x\rangle$. We then let $W_{n}^{ \pm}$be the skew-Hermitian space such that $\left(W_{n}^{ \pm}\right)^{\prime} \simeq V_{n}^{ \pm}$. If $n$ is even, this space is independent of the choice of $\delta$, while, if $n$ is odd, it depends on that choice. 
Now suppose again that $\pi$ is a supercuspidal representation of $G(W)$, fix a character $\chi$ of $E^{\times}$with $\left.\chi\right|_{F^{\times}}=\epsilon_{E / F}^{m_{00}}, m_{00}=0,1$, and consider the collection of theta lifts $\theta_{\chi}\left(\pi, V_{m}^{ \pm}\right)$as $V_{m}^{ \pm}$runs over a fixed Witt tower $\left\{V_{m}^{ \pm}\right\}$, with $m$ of the same parity as $m_{00}$. Let $m_{0}^{ \pm}(\chi)$ be the smallest $m$ such that $\theta_{\chi}\left(\pi, V_{m}^{ \pm}\right) \neq 0$. Then it is known [29] that $\theta_{\chi}\left(\pi, V_{m_{0}^{ \pm}(\chi)}^{ \pm}\right)$is a supercuspidal representation of $G\left(V_{m_{0}^{ \pm}}^{ \pm}\right)$and that, for $m=m_{0}^{ \pm}(\chi)+2 t, t>0$, the representation $\theta_{\chi}\left(\pi, V_{m}^{ \pm}\right)$is non-zero and not supercuspidal. Thus, if $m_{00}$ has the same parity as $n=\operatorname{dim}_{E} W$, theta dichotomy implies that one element of the set $\left\{m_{0}^{+}(\chi), m_{0}^{-}(\chi)\right\}$ is $\leq n$ (the 'early' tower for $\pi$ and $\chi$ ) while the other is $>n$ (the 'late' tower for $\pi$ and $\chi$ ). If, say, $m_{0}^{+}(\chi) \leq n$ and $n_{1}:=m_{0}^{-}(\chi)>n$, then the representation $\pi_{1}=\theta_{\chi}\left(\pi, V_{n_{1}}^{-}\right)$is a supercuspidal representation of $G\left(V_{n_{1}}^{-}\right)$, and we can repeat the construction, considering the theta lifts of $\pi_{1}$ to the two skew-Hermitian Witt towers. In the following statement we identify Hermitian and skew-Hermitian forms $W_{m}^{ \pm}$and $V_{n}^{ \pm}$, as above, and we again take $\chi=\chi_{1}=\chi_{2}$. By the procedure just described, we obtain:

Chains of supercuspidals (Section 5). For any supercuspidal representation $\pi$ of $G(W)$, and choice of character $\chi$ of $E^{\times}$with $\left.\chi\right|_{F^{\times}}=\epsilon_{E / F}^{n}, n=\operatorname{dim}_{E} W$, there exists a sequence of dimensions $n=n_{0}<n_{1}<n_{2}<\cdots<n_{i}<\ldots$ and supercuspidal representations $\pi=\pi_{0}, \pi_{1}, \ldots, \pi_{i}, \ldots$ of $G\left(V_{n_{i}}^{ \pm}\right)$such that

$$
\theta_{\chi}\left(\pi_{i}, V_{n_{i+1}}^{ \pm}\right)=\pi_{i+1}
$$

Here, for example, if $W \simeq W_{n}^{+}$, the sequence of signs is

$$
+, \epsilon,-,-\epsilon,+, \ldots
$$

For example, in the case $n=1$, the first step in such a chain can be found in Rogawski [41]. This is explained in detail in section 7. In general, Speculation 7.5 suggests that

$$
m_{0}^{+}(\chi)+m_{0}^{-}(\chi)=2 n+2
$$

If we assume that $\theta_{\chi}\left(\pi, V_{n}^{ \pm}\right)$is also supercuspidal, i.e., that $\pi$ does not come from a smaller unitary group via the theta correspondence, then Speculation 7.5 implies that the sequence of dimensions will be

$$
n, n+2, n+6, n+12, \ldots
$$

Relation (0.11) and (0.12) have now been proved for $\pi$ supercuspidal by Rallis and the second author [24]. We note that there is an analogous construction when the Hermitian and skew-Hermitian spaces have dimensions of opposite parity. This is described at the end of section 7. Moreover, a similar phenomenon occurs for reductive dual pairs of type $(S p, O)$.

In section 7 , we combine dichotomy with several other basic principles and conjectures to give some speculations about the functoriality properties of the theta correspondence for unitary groups. It is known, for example, that the theta correspondence does not always preserve L-packets, whereas, according to conjectures of Adams [1], which are based on fairly extensive evidence in the archimedean 
case, the grouping of representations into A-packets should be preserved. It seems that theta dichotomy is at the root of the failure of functoriality on the level of L-packets. For example, for $n$ odd, the L-group will not distinguish the groups $G\left(V_{n}^{+}\right)$and $G\left(V_{n}^{-}\right)$- they can be canonically identified as groups-whereas the theta correspondence behaves very differently in the two towers. The speculations of section 7 suggest that it should be possible, nonetheless, to predict the behavior of L-packets, in the extended sense [48], under the correspondence. In particular, we give a conjecture, Speculation 7.7, about the L-parameters of the supercuspidals in a chain. Specifically, let $W_{F}$ be the Weil group of $F$, let $W_{F}^{\prime}=S L_{2}(\mathbb{C}) \times W_{F}$, and let $\varphi_{0}: W_{F}^{\prime} \longrightarrow{ }^{L} G\left(W_{n}\right)$ be the L-parameter for $\pi=\pi_{0}$. Assume, as above, that $\pi$ does not come from a smaller unitary group via the theta correspondence. Then the L-parameter for $\pi_{r}$ should be

$$
\varphi_{r}=\varphi_{0} \oplus \chi\left(\rho_{2} \oplus \rho_{4} \oplus \cdots \oplus \rho_{2 r}\right),
$$

where $\rho_{b}$ is the $(b-1)$-st symmetric power of the standard representation of $S L_{2}(\mathbb{C})$.

For a given supercuspidal $\pi$ of $G\left(W_{n}\right)$, the dichotomy principle says that, if $\epsilon= \pm 1$ is the sign for the early tower, i.e., if $\theta_{\chi}\left(\pi, V_{n}^{\epsilon}\right) \neq 0$, then $m_{0}^{\epsilon}=n-2 r$, for some $r \geq 0$. It turns out that how 'early' $\pi$ occurs is reflected in the L-factor. For any character $\chi$ of $E^{\times}$, let $\check{\chi}(x)=\chi(\bar{x})^{-1}$. The following result is the analogue for unitary groups of an unpublished result of Rallis and the second author for dual pairs of type $(S p, O)$.

Early lifts and poles (Theorem 6.2). For a supercuspidal representation $\pi$ of $G(W)$, for any character $\chi$ of $E^{\times}$, and for $s \in \mathbb{C}$, assume that the pair $(s, \chi)$ is normalized as at the beginning of section 6 .

(i) If $\chi \neq \check{\chi}$, then $L_{P S R}(s, \pi, \chi)=1$.

(ii) If $\chi=\check{\chi}$, and $\left.\chi\right|_{F^{\times}}=\epsilon_{E / F}^{n}$ let

$$
m_{0}=m_{0}(\chi)=\min \left\{m_{0}^{+}(\chi), m_{0}^{-}(\chi)\right\}=n-2 r .
$$

Then, if $r>0$,

$$
L_{P S R}\left(s, \pi^{\vee}, \chi\right)=\left(1-q_{E}^{-\left(s+\frac{1}{2}\right)}\right)^{-1}\left(1-q_{E}^{-\left(s+\frac{3}{2}\right)}\right)^{-1} \ldots\left(1-q_{E}^{-\left(s+r-\frac{1}{2}\right)}\right)^{-1} .
$$

If $r=0, L_{P S R}\left(s, \pi^{\vee}, \chi\right)=1$.

(iii) If $\chi=\check{\chi}$, and $\left.\chi\right|_{F^{\times}}=\epsilon_{E / F}^{n-1}$ let

$$
m_{0}=m_{0}(\chi)=\min \left\{m_{0}^{+}(\chi), m_{0}^{-}(\chi)\right\}=n-2 r+1 .
$$

Then, if $r>0$,

$$
L_{P S R}\left(s, \pi^{\vee}, \chi\right)=\left(1-q_{E}^{-s}\right)^{-1}\left(1-q_{E}^{-(s+1)}\right)^{-1} \ldots\left(1-q_{E}^{-(s+r-1)}\right)^{-1} .
$$

If $r=0, L_{P S R}\left(s, \pi^{\vee}, \chi\right)=1$.

Note that in case (iii), any representation of $G(W)$ has $\theta_{\chi}\left(\pi, V_{n+1}^{ \pm}\right) \neq 0$ for at least one choice of \pm , and this should usually be the first non-zero theta lift, i.e., one should usually have $r=0$. Similarly, in case (ii), one expects to have $r=0$.

The $\mathrm{L}$ and $\epsilon$-factors occuring in our results are those defined by Piatetski-Shapiro and Rallis. One would like to prove the following: 
Comparison Conjecture. For all irreducible admissible representations $\pi$ of $G(W)$, and characters $\chi$ of $E^{\times}$, let $B C(\pi)$ be the base change of $\pi$ to $G L_{n}(E)$. Then

$$
L_{P S R}(s, \pi, \chi)=L(s, B C(\pi) \otimes \chi) .
$$

If $n$ is odd, then

$(0.19$ (odd) $)$

$$
\epsilon_{P S R}(s, \pi, \chi, \psi ; W)=\chi(-\operatorname{det} W)|\operatorname{det} W|_{E}^{s-\frac{1}{2}} \omega_{\pi}(-1) \epsilon\left(s, B C(\pi) \otimes \chi, \psi_{E}\right) .
$$

If $n$ is even, let $\epsilon(W)=+1$ if $G(W)$ is quasi-split, $\epsilon(W)=-1$ if not. Then

$\left(0.19\right.$ (even)) $\epsilon_{P S R}(s, \pi, \chi, \psi ; W)=\epsilon(W)|\operatorname{det} W|_{E}^{s-\frac{1}{2}} \omega_{\pi}(-1) \epsilon\left(s, B C(\pi) \otimes \chi, \psi_{E}\right)$.

Here, if the skew-Hermitian form on $W$ has matrix $\delta \mathbf{a}$, with a Hermitian, then $\operatorname{det} W=\delta^{n} \operatorname{det} \mathbf{a}$.

The base change involved here is well known for $n=1$, and is due to Rogawski [41] for $n=2$ and 3. In general, it can be defined implicitly using the global method of [5], although it is difficult to verify that the resulting local base change is independent of its imbedding into a global situation.

In the case $n=1$ we verify this conjecture in section 8 , Proposition 8.4 , by an amusing calculation. With this additional information, our general results on theta and epsilon dichotomy specialize to a new proof of the local results of Rogawski [42] and Moen [30] for the dual pair $(U(1), U(1))$. Our proof has the advantage of being purely local, and it does not involve any calculations with Gauss sums.

In the case $n=2$, our results (almost) specialize to those of [15], except for the fact that things in [15] are expressed in terms of the similitude group $G U(2)$. Again, the methods of the present paper yield new proofs, although we do not prove the comparison conjecture for $n=2$.

Finally, it should be noted that the global results of Rogawski [42], GelbartRogawski [6] for $n=1$ and of Harris [15, 16] for $n=2$, as well as the global characterization of 'early' theta lifts via poles in the case of $(S p, O)$ dual pairs [23], should have generalizations to unitary groups for all $n$, by methods which are by now standard, based on the local results of this paper and a suitable generalization of the Siegel-Weil formula [23] to the unitary case.

We now give a section by section summary. One of the main technical difficulties involved in the study of the theta correspondence for unitary groups is the non-uniqueness of the splitting homomorphism (0.2). In particular, one has to keep careful track of the behavior of the splitting when restricting to subgroups, e.g., in a seesaw construction. In section 1 and in the appendix, we describe, in (1.16) and the discussion preceding it, how to associate a splitting to a pair of characters, as above, and we make a fairly thorough analysis of the compatibility of the splittings, so defined, with restrictions to subgroups, direct sums, etc. These results, which are based on [22], are essential to what follows and will be fundamental in future applications as well. In section 2, we consider the Weil representation defined using the splitting homomorphism defined by $\chi$. We determine its behavior under pullback via doubling and under the Moeglin-Vigneras-Waldsurger (MVW) involution (Proposition 2.2). If $W,\langle$,$\rangle is a skew-Hermitian space, as above, let W_{-}=W$ 
with skew-Hermitian form $-\langle$,$\rangle , and let$

$$
i: G(W) \times G(W)=G(W) \times G\left(W_{-}\right) \longrightarrow G\left(W+W_{-}\right)
$$

be the natural homomorphism. For a Hermitian space $V$, and choice of $\chi_{1}$, we have Weil representations $\omega_{V, W, \chi}$ of $G(V) \times G(W)$ and $\omega_{V, W+W_{-}, \chi}$ of $G(V) \times$ $G\left(W+W_{-}\right)$, and the latter can be realized on the Schwartz space $S\left(V^{n}\right)$ with $G(V)$ acting by the twist by $\chi$ of its natural linear action. Let $R_{n}(V, \chi)$ be the maximal quotient of $S\left(V^{n}\right)$ on which $G(V)$ acts by a multiple of $\chi$. This representation of $G\left(W+W_{-}\right) \simeq U(n, n)$ is a submodule of a certain degenerate principal series representation $I_{n}(s, \chi)$, the structure of which was completely determined in [25]. For example, if $m=n$,

$$
I_{n}(0, \chi)=R_{n}\left(V_{n}^{+}, \chi\right) \oplus R_{n}\left(V_{n}^{-}, \chi\right),
$$

where $V_{n}^{ \pm}$is as above. The relevant results of [25] are summarized in Proposition 4.1. In section 3, we prove that for an irreducible admissible representation $\pi$ of $G(W)$,

$$
\Theta_{\chi}(\pi, V) \neq 0 \quad \Longleftrightarrow \quad \operatorname{Hom}_{G(W) \times G(W)}\left(R_{n}(V, \chi), \pi \otimes\left(\chi \pi^{\vee}\right)\right) \neq 0,
$$

(Proposition 3.1). The existence of this sort of relation is due to Rallis [36]. Next, in section 4 , we prove:

Key Theorem (Theorem 4.3). (i) If $\pi$ is any irreducible admissible representation of $G(W)$, then, for all $s$,

$$
\operatorname{dim} \operatorname{Hom}_{G(W) \times G(W)}\left(I_{n}(s, \chi), \pi \otimes\left(\chi \pi^{\vee}\right)\right) \geq 1 .
$$

(ii) If $\pi$ is supercuspidal, then, for all $s$,

$$
\operatorname{dim} \operatorname{Hom}_{G(W) \times G(W)}\left(I_{n}(s, \chi), \pi \otimes\left(\chi \pi^{\vee}\right)\right)=1 .
$$

Note that it is proved in [32] that, for any $\pi$, the dimension of this space of homomorphisms is at most 1 for almost all $s$. Theta dichotomy for supercuspidals follows immediately from the combination of $(0.21),(0.22)$, and $(0.24)$ at $s=0$. It has long been held by Rallis and the second author that (0.24) should hold for all $\pi$, and this would imply theta dichotomy in general. In fact, we prove (0.24) at $s=0$ for 'most' representations (Theorem 4.3), and hence obtain theta dichotomy for these (Corollary 4.4).

In section 5 , we discuss the chains of supercuspidals in detail.

In section 6 , we first recall the construction of $\mathrm{L}$ and $\epsilon$-factors by the PiatetskiShapiro, Rallis doubling method of [31], [32], [33]. The main new ingredient here is an explicit calculation (Proposition 6.3) of the normalizing factor for the intertwining operator $M(s, \chi),(6.1)$, which occurs in the functional equation, (iv) of Proposition 6.6 and (6.18), of the family of zeta integrals. This calculation, which is based on the technique of [44], allows us to give a precise definition of the normalized $\Gamma$ factor $\Gamma^{*}(s, \pi, \chi, \psi)$ (Proposition 6.6), and hence of the $\epsilon$-factor, (6.19). It should be noted that, when $n=\operatorname{dim}_{E} W$ is odd, the factor $\epsilon(s, \pi, \chi, \psi)=\epsilon_{P S R}(s, \pi, \chi, \psi ; W)$ depends on $W$ (Proposition 6.11). Note that the normalized intertwining operator satisfies the functional equation

$$
M^{*}(-s, \check{\chi}) \circ M^{*}(s, \chi)=1 .
$$


The key idea in the proof of epsilon dichotomy is then that, for a supercuspidal representation $\pi$ of $G(W)$, the unique intertwining map of (0.24), at $s=0$, is supported on the summand in (0.21) on which the sign of the normalized intertwining operator $M^{*}(0, \chi)$ agrees with the root number $\epsilon_{P S R}\left(\frac{1}{2}, \pi, \chi, \psi ; W\right)$. Note that, by (0.25), $M^{*}(0, \chi)^{2}=1$. The eigenvalue of $M^{*}(0, \chi)$ on the summand $R_{n}(V, \chi)$ is given in Proposition 6.8. Finally, for supercuspidal representations, we establish the relation between poles of the L-factor $L_{P S R}(s, \pi, \chi)$ and early local theta lifts (Theorem 6.2) described above.

In section 7, we attempt to give a picture of the behavior of L-packets under the local theta correspondence. Our results on dichotomy and poles of L-factors provide some important clues, and we make a series of speculations (Speculations 7.3-7.7) about how the local theta correspondence for unitary groups should look. Among other things, the validity of these speculations would show that theta dichotomy forces L-packets to be broken up in the correspondence (failure of functoriality), but that this phenomenon takes place in a precisely controlled way which is compatible with Adams' conjectures about the preservation of A-packets [1]. Some of the speculations may be accessible at the moment, while others assume the local Langlands conjecture for general unitary groups!

In section 8 , we give a rather self contained treatment of the case $n=1$, a case which is not so trivial, as several authors have observed. There we prove $n=1$ versions of the key comparison results (0.18) and (0.19) for $\mathrm{L}$ and $\epsilon$-factors. There is some chance that our method can be extended to arbitrary $n$, but some new ideas will be needed.

The results of this paper have been in the mill for a long time and the authors owe thanks to a long list of patient benefactors. The first and second authors would particularly like to thank Université de Paris VII and the Isaac Newton Institute, where joint work on this project was undertaken for the months of November 1992 and April 1993, respectively. The second author would like to thank the T.I.F.R. in Bombay for providing an excellent working environment for the month of January, 1994. He is also indebted to the General Research Board of the University of Maryland for a Research Semester Grant for the fall of 1992 and to the University of Maryland for Sabbatical leave during the spring semester of 1993.

In the course of this work, we have benefited from the ideas and suggestions of J. Adams, S. Gelbart, B. Gross, S. Rallis, D. Prasad, and J. Rogawski. In fact, the main impetus to finish the present version was provided by some stimulating conversations with Steve Gelbart during the spring (at Maryland) and summer (at Yale) of 1993. Also, the analogues, in the $(S p(n), O(2 r))$ case, of some of the speculations of section 7 will appear in some joint work of the second author with D. Prasad.

Finally, the first two authors wish to acknowledge support of Grants (DMS9203142 and DMS-9302539, respectively) from the National Science Foundation.

Notation. Let $F$ be a non-archimedean local field of characteristic 0 with ring of integers $\mathcal{O}_{F}$ and maximal ideal $\mathcal{P}$. Let $E / F$ be a quadratic extension with associated quadratic character $\epsilon_{E / F}$. Let $\tau: x \mapsto \bar{x}$ denote the non-trivial Galois automorphism of $E$ over $F$. We will sometimes write $\bar{x}=x^{\tau}$. Let $N_{E / F}$ and $\operatorname{tr}_{E / F}$ be the norm and trace maps from $E$ to $F$. We choose an element $\delta \in E^{\times}$such that $\bar{\delta}=-\delta$. Then $\delta^{2}=\Delta \in F^{\times}$and $\epsilon_{E / F}(x)=(x, \Delta)_{F}$ is the quadratic character of $F^{\times}$associated to $E / F$ by local class field theory. Here $(x, y)_{F}$ is the Hilbert symbol 
for $F$. We let $E^{1}$ be the kernel of the norm map and recall that the sequence

$$
\begin{aligned}
1 \longrightarrow F^{\times} \longrightarrow E^{\times} & \longrightarrow E^{1} \longrightarrow 1 \\
x & \mapsto x / \bar{x}
\end{aligned}
$$

is exact. If $\mu$ is a character of $E^{1}$ we let

$$
\tilde{\mu}(x)=\mu(x / \bar{x}) .
$$

Similarly, if $\chi$ is a character of $E^{\times}$, we will sometimes write $\chi^{o}$ for its restriction to $F^{\times}$.

Fix, once and for all, a non-trivial additive character $\psi$ of $F$ and extend it to $E$ via the trace. We will let $n(\psi)$ be the largest integer $n$ such that $\psi$ is trivial on $\mathcal{P}^{-n}$. We say that $\psi$ is unramified if $n(\psi)=0$. Also, for $a \in F^{\times}$, let $\psi_{a}(x)=\psi(a x)$.

We will usually write $V$ (resp. $W$ ) for an $E$ vector space with a Hermitian (resp. skew-Hermitian) form $(, \quad)$ (resp. $\langle$,$\rangle ). All such forms are assumed to be non-$ degenerate. We take $V$ to be a right $E$ vector space, with $\operatorname{dim}_{E} V=m$, and hence have

$$
\left(v_{1} a, v_{2} b\right)=\bar{a}\left(v_{1}, v_{2}\right) b,
$$

for $a, b \in E$. Similarly, $W$ will be a left $E$ vector space, with $\operatorname{dim}_{E} W=n$, and

$$
\left\langle a w_{1}, b w_{2}\right\rangle=a\left\langle w_{1}, w_{2}\right\rangle \bar{b}
$$

These conventions come into play in the definitions of tensor products. Also, if $W$ is a given skew-Hermitian space and if $\delta$ is chosen as above, then we can define a Hermitian space $W^{\prime}$ by $W^{\prime}=W$ (viewed as a right vector space via $w a=a w$ ) and

$$
\left(w_{1}, w_{2}\right)=\delta^{-1}\left\langle w_{2}, w_{1}\right\rangle
$$

Thus there will be a symmetry to many of our constructions, but there may be a dependence on the choice of $\delta$.

For a Hermitian space $V$ or skew-Hermitian space $W, G(V)$ and $G(W)$ will denote the isometry groups. Note that, if $a \cdot V, a \in F^{\times}$is the space $V$ with Hermitian form $a($, $)$, then $G(a \cdot V)=G(V)$.

For a fixed dimension $m$, there are two isomorphism classes of non-degenerate Hermitian forms over $E$, and these are distinguished by the quantity $\operatorname{det} V \in$ $F^{\times} / N E^{\times}$. If $m=\operatorname{dim}_{E} V=1$, the two classes of Hermitian forms are given by $V_{1}^{ \pm}=E$ with $(x, y)_{+}=\bar{x} y$ and $(x, y)_{-}=a \bar{x} y$, where $a \in F^{\times}-N E^{\times}$, i.e., $\epsilon_{E / F}(a)=-1$. In general, for $m=2 r+1$ odd, the two classes of Hermitian forms are given by

$$
V_{m}^{ \pm}=V_{1}^{ \pm}+V_{r, r}
$$

where $V_{r, r}$ is the Hermitian space of dimension $2 r$ with Hermitian form given by

$$
\left(\begin{array}{cc}
0 & 1_{r} \\
1_{r} & 0
\end{array}\right)
$$


for a suitable choice of basis. Note that

$$
\epsilon_{E / F}\left((-1)^{\frac{m(m-1)}{2}} \operatorname{det} V_{m}^{ \pm}\right)= \pm 1 .
$$

Also, if $a \in F^{\times}$with $\epsilon_{E / F}(a)=-1$, then

$$
a \cdot V_{2 r+1}^{ \pm} \simeq V_{2 r+1}^{\mp} .
$$

If $m$ is even, we again denote representatives of the two classes of Hermitian forms by $V_{m}^{+}$and $V_{m}^{-}$, where the sign \pm is determined by (n.7). Note that $V_{2 r}^{+}=V_{r, r}$; we refer to this space as the split space. If $E / F$ is unramified, we refer to the space $V_{m}^{+}$as the unramified Hermitian space of dimension $m$.

For a fixed choice of $\delta$, we can transfer this classification to skew-Hermitian spaces by setting $W=W_{n}^{ \pm}$if $W^{\prime}=V_{n}^{ \pm}$. If $n=\operatorname{dim}_{E} W$ is even, the resulting notation is independent of $\delta$ and $W_{2 r}^{+}$, the split space, has matrix

$$
\left(\begin{array}{cc}
0 & 1_{r} \\
-1_{r} & 0
\end{array}\right)
$$

for a suitable choice of basis. On the other hand, if $n$ is odd, then the space $W=W_{n}^{+}$depends on the choice of $\delta$, via (n.8)! This fact must be reflected in our formulas.

The groups $G\left(V_{m}^{+}\right)$and $G\left(W_{n}^{+}\right)$are quasisplit for all $m$ and $n$, while the groups $G\left(V_{2 r}^{-}\right)$and $G\left(W_{2 r}^{-}\right)$are not.

For an admissible representation $\sigma$ of a p-adic group $G$, we let $\sigma^{\vee}$ denote the contragredient. Our induced representations are always normalized, i.e. are (pre-)unitary for (pre-)unitary induction data.

We write $\mu_{n}$ for the group of $\mathrm{n}$-th roots of unity.

For any non-trivial additive character $\eta$ of $F$ and $a \in F^{\times}$, we will let

$$
\gamma_{F}(a, \eta)=\frac{\gamma_{F}\left(\eta_{a}\right)}{\gamma_{F}(\eta)} \in \mu_{4}
$$

where $\gamma_{F}(\eta) \in \mu_{8}$ is the Weil index of the character of second degree $x \mapsto \eta\left(x^{2}\right)$. Here $\left(\eta_{a}\right)(x)=\eta(a x)$. Recall that

$$
\gamma_{F}(a b, \eta)=(a, b)_{F} \gamma_{F}(a, \eta) \gamma_{F}(b, \eta) .
$$

\section{$\S 1$. Cocycles And SPlittings}

In this section we record some essential facts concerning the restriction of the metaplectic covering to a dual pair of unitary groups. Of particular importance (and delicacy) is the behavior of these splittings for the seesaw dual pairs of the doubling construction.

Let $W,\langle$,$\rangle (resp. V,()$,$) be a non-degenerate skew-Hermitian (resp. Hermit-$ ian) space over $E$ with $\operatorname{dim}_{E} W=n\left(\right.$ resp. $\left.\operatorname{dim}_{E} V=m\right)$. Let

$$
\mathbb{W}=V \otimes_{E} W
$$

with the symplectic form

$$
\left\langle\langle,\rangle=\frac{1}{2} \operatorname{tr}_{E / F}\left((,) \otimes\langle,\rangle^{\tau}\right),\right.
$$

as usual. Let

$$
1 \longrightarrow \mathbb{C}^{1} \longrightarrow M p(\mathbb{W}) \longrightarrow S p(\mathbb{W}) \longrightarrow 1
$$


denote the metaplectic cover of $S p(\mathbb{W})$, which we view as an extension of $S p(\mathbb{W})$ by $\mathbb{C}^{1}$, in order to have more room to modify our cocycles (cf. [22]). It should be noted that, in contrast to the usual twofold covering, this group now has non-trivial characters.

We want to construct the Weil representation for the dual reductive pair $U(V)$, $U(W)$ in $S p(\mathbb{W})$, and, in particular, to be careful about the dependence of this representation on a choice of a splitting of the restriction of the metaplectic cover $M p(\mathbb{W}) \longrightarrow S p(\mathbb{W})$ to $U(V)$ and to $U(W)$. For convenience, we write $G(V)=U(V)$ and $G(W)=U(W)$, etc. for the unitary groups involved.

We will begin with the group $G(W)$. For a fixed Hermitian space $V$, let

$$
\iota_{V}: G(W) \longrightarrow S p(\mathbb{W})
$$

be the natural homomorphism. If $\operatorname{dim}_{E} V=m$, we choose a character $\chi=\chi_{V}$ of $E^{\times}$such that

$$
\left.\chi_{V}\right|_{F^{\times}}=\epsilon_{E / F}^{m},
$$

where $\epsilon_{E / F}$ is the quadratic character associated to the extension $E / F$. This choice determines a lift

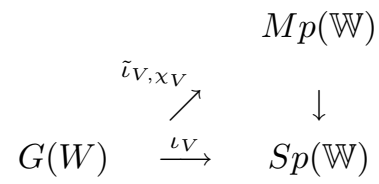

by a procedure which we will review in a moment. Note that two choices $\chi_{1}$ and $\chi_{2}$ of $\chi_{V}$ differ by a character $\mu$ of $E^{\times}$which is trivial on $F^{\times}$, i.e., $\chi_{2}=\mu \chi_{1}$. Then $\mu$ defines a character $\mu^{\prime}$ on $E^{1}$ by

$$
\mu^{\prime}\left(\frac{x}{\bar{x}}\right)=\mu(x)
$$

and we have

$$
\tilde{\iota}_{V, \chi_{2}}=\left(\mu^{\prime} \circ \operatorname{det}\right) \cdot \tilde{\iota}_{V, \chi_{1}},
$$

where $\mu^{\prime} \circ$ det takes values in the central $\mathbb{C}^{1}$ in $M p(\mathbb{W})$.

We now describe the construction of $\tilde{\iota}_{V, \chi}$, following [22]. Let $W_{-}$denote the space $W$ with the skew-Hermitian form $-\langle$,$\rangle . Note that the identity map on W$ gives an anti-isometry $W=W_{-}$. Then let

$$
\mathbb{W}_{-}=V \otimes_{E} W_{-}
$$

so that $\mathbb{W}=\mathbb{W}_{-}$(an anti-isometry). Let $\iota_{V}$ be the natural embedding of $G(W)$ into $S p(\mathbb{W})$. We then obtain a commutative diagram

$$
\begin{array}{ccc}
G\left(W+W_{-}\right) & \stackrel{\iota_{V}}{\longrightarrow} & S p\left(\mathbb{W}+\mathbb{W}_{-}\right) \\
\uparrow i & & \uparrow i \\
G(W) \times G(W) & \stackrel{\iota_{V} \times \iota_{V}}{\longrightarrow} & S p(\mathbb{W}) \times S p(\mathbb{W}) \\
\uparrow \Delta & & \uparrow \Delta \\
G(W) & \longrightarrow & S p(\mathbb{W})
\end{array}
$$


Here we identify $G(W)$ and $G\left(W_{-}\right)$(resp. $S p(\mathbb{W})$ and $S p\left(\mathbb{W}_{-}\right)$) via the identity (anti-isometry) of $W$ and $W_{-}$(resp. $\mathbb{W}$ and $\left.\mathbb{W}_{-}\right)$. The metaplectic covering $M p\left(\mathbb{W}+\mathbb{W}_{-}\right)$splits over each of these subgroups [22], and we want to determine the compatibilities among the splittings.

Let

$$
W+W_{-}=X+Y
$$

be the polarization of the split skew-Hermitian space $W+W_{-}$where $X$ is the graph of minus the identity map from $W$ to $W$, and $Y$ is the graph of the identity map. This choice of polarization induces a polarization $\mathbb{X}+\mathbb{Y}$ of $\mathbb{W}+\mathbb{W}$, with $\mathbb{X}=V \otimes_{E} X$ and $\mathbb{Y}=V \otimes_{E} Y$.

We fix a non-trivial additive character $\psi$ of $F$. The choice of maximal isotropic subspace $\mathbb{Y}$ in $\mathbb{W}+\mathbb{W}$ - then determines an isomorphism

$$
M p(\mathbb{W}+\mathbb{W}-) \simeq S p\left(\mathbb{W}+\mathbb{W}_{-}\right) \times \mathbb{C}^{1}
$$

as sets [40]. Here the multiplication on the right hand side is described by the cocycle $c_{\mathbb{Y}}\left(g_{1}, g_{2}\right)$ given by the Leray invariant [22], [40]

$$
c_{\mathbb{Y}}\left(g_{1}, g_{2}\right)=\gamma_{F}\left(\eta \circ L\left(\mathbb{Y}, \mathbb{Y} g_{2}^{-1}, \mathbb{Y} g_{1}\right)\right) \text {. }
$$

Here $\eta=\frac{1}{2} \psi$, as in [22]. Moreover, having chosen the character $\chi$ with $\chi_{F^{\times}}=\epsilon_{E / F}^{m}$, $m=\operatorname{dim}_{E} V$, as above, we also obtain an explicit homomorphism [22]

$$
\begin{aligned}
\tilde{\iota}_{V, \chi}: G\left(W+W_{-}\right) & \longrightarrow M p\left(\mathbb{W}+\mathbb{W}_{-}\right) \simeq S p\left(\mathbb{W}+\mathbb{W}_{-}\right) \times \mathbb{C}^{1} \\
g & \mapsto \tilde{\iota}_{V, \chi}(g) \simeq\left(\iota_{V}(g), \beta_{V, \chi}(g)\right),
\end{aligned}
$$

lifting $\iota_{V}$. Here

$$
\beta_{V, \chi}(g)=\chi_{V}(x(g)) \gamma_{F}(\eta \circ R V)^{-j(g)},
$$

where the notation is as in [22]. In particular,

$$
\gamma_{F}(\eta \circ R V)=(\Delta, \operatorname{det} V)_{F} \gamma_{F}(-\Delta, \eta)^{m} \gamma_{F}(-1, \eta)^{-m}
$$

Now the inverse image in $M p\left(\mathbb{W}+\mathbb{W}_{-}\right)$of the image of $S p(\mathbb{W}) \times 1$ (resp. $1 \times$ $S p(\mathbb{W}))$ in $S p(\mathbb{W}+\mathbb{W}-)$ is isomorphic to $M p(\mathbb{W})$. This isomorphism is canonical if we specify its restriction to the central $\mathbb{C}^{1}$. We choose a lift $\tilde{i}$ of $i$ so that

$$
\tilde{i}: M p(\mathbb{W}) \times M p(\mathbb{W}) \longrightarrow M p(\mathbb{W}+\mathbb{W}-)
$$

restricts to the map

$$
\begin{aligned}
\mathbb{C}^{1} \times \mathbb{C}^{1} & \longrightarrow \mathbb{C}^{1} \\
\left(\epsilon_{1}, \epsilon_{2}\right) & \mapsto \epsilon_{1} \bar{\epsilon}_{2}
\end{aligned}
$$

on the central $\mathbb{C}^{1}$ 's.

Then the restriction of $\tilde{\iota}_{V, \chi}$ to $G(W) \times 1$ yields a splitting homomorphism

$$
\tilde{\iota}_{V, \chi}: G(W) \longrightarrow M p(\mathbb{W})
$$


lifting $\iota_{V}$. Similarly, the restriction of $\tilde{\iota}_{V, \chi}$ to $1 \times G(W)$ yields a splitting homomorphism

$$
\tilde{\iota}_{V, \chi,-}: G(W) \longrightarrow M p(\mathbb{W})
$$

Thus we have a diagram

$$
\begin{array}{ccc}
G\left(W+W_{-}\right) & \stackrel{\tilde{\iota}_{V, \chi}}{\longrightarrow} & M p\left(\mathbb{W}+\mathbb{W}_{-}\right) \\
\uparrow i & & \uparrow \tilde{i} \\
G(W) \times G(W) & \underset{\tilde{\iota}_{V, \chi} \times \tilde{\iota}_{V, \chi,-}}{\longrightarrow} & M p(\mathbb{W}) \times M p(\mathbb{W})
\end{array}
$$

lifting the upper part of (1.10).

We first determine the relation between $\tilde{\iota}_{V, \chi}$ and $\tilde{\iota}_{V, \chi,-}$.

Lemma 1.1. $\tilde{\iota}_{V, \chi,-}=\chi^{-1} \cdot \tilde{\iota}_{V, \chi}$.

Proof. Let

$$
c: \mathbb{W}+\mathbb{W}-\longrightarrow \mathbb{W}+\mathbb{W}_{-}
$$

be the anti-isometry induced by switching $W$ and $W_{-}$, i.e., sending the pair $\left(w_{1}, w_{2}\right)$ $\in W \oplus W_{-}$to $\left(w_{2}, w_{1}\right) \in W \oplus W_{-}$. This map acts by +1 on $\mathbb{Y}$ and by -1 on $\mathbb{X}$. Thus $-c$ has matrix $\left(\begin{array}{cc}1 & 0 \\ 0 & -1\end{array}\right)$ on $\mathbb{X}+\mathbb{Y}$. Conjugation by $-c$ induces an automorphism $s w$ of $S p(\mathbb{W}+\mathbb{W}-)$ such that the diagram

$$
\begin{array}{rcc}
S p\left(\mathbb{W}+\mathbb{W}_{-}\right) & \stackrel{s w}{\longrightarrow} & S p\left(\mathbb{W}+\mathbb{W}_{-}\right) \\
\uparrow i & \uparrow i \\
S p(\mathbb{W}) \times S p(\mathbb{W}) & \stackrel{\text { switch }}{\longrightarrow} & S p(\mathbb{W}) \times S p(\mathbb{W}) \\
\uparrow \iota_{V} \times \iota_{V} & & \uparrow \iota_{V} \times \iota_{V} \\
G(W) \times G(W) & \stackrel{\text { switch }}{\longrightarrow} & G(W) \times G(W)
\end{array}
$$

commutes.

The following result is easily checked.

Lemma 1.2. There is a unique lift sww, which restricts to complex conjugation on the central $\mathbb{C}^{1}$, of the automorphism sw of $S p(\mathbb{W}+\mathbb{W}-)$ given by conjugation by $\left(\begin{array}{cc}1 & 0 \\ 0 & -1\end{array}\right)$. For the isomorphism

$$
M p\left(\mathbb{W}+\mathbb{W}_{-}\right) \simeq S p\left(\mathbb{W}+\mathbb{W}_{-}\right) \times \mathbb{C}^{1}
$$

determined by $\mathbb{Y}$, siw is given by:

$$
(g, \epsilon) \mapsto(\operatorname{Ad}(c) g, \bar{\epsilon}) .
$$


Moreover, the diagram

$$
\begin{aligned}
M p\left(\mathbb{W}+\mathbb{W}_{-}\right) & \stackrel{s \tilde{w}}{\longrightarrow} & M p\left(\mathbb{W}+\mathbb{W}_{-}\right) \\
\uparrow \tilde{i} & & \uparrow \tilde{i} \\
M p(\mathbb{W}) \times M p(\mathbb{W}) & \stackrel{\text { switch }}{\longrightarrow} & M p(\mathbb{W}) \times M p(\mathbb{W}) \\
\uparrow \tilde{\iota}_{V, \chi} \times \tilde{\iota}_{V, \chi,-} & & \uparrow \tilde{\iota}_{V, \chi} \times \tilde{\iota}_{V, \chi,-} \\
G(W) \times G(W) & \stackrel{\text { switch }}{\longrightarrow} & G(W) \times G(W)
\end{aligned}
$$

commutes.

For $g \in G(W)$, we now compute:

$$
\begin{aligned}
s \tilde{w} \circ \tilde{i}\left(1, \tilde{\iota}_{V, \chi,-}(g)\right) & =s \tilde{w} \circ \tilde{\iota}_{V, \chi}(i(1, g)) \\
& =s \tilde{w}\left(\iota_{V}(i(1, g)), \beta_{V, \chi}(i(1, g))\right) \\
& =\left(\iota_{V}(i(g, 1)), \overline{\beta_{V, \chi}(i(1, g))}\right) .
\end{aligned}
$$

We then use the following relations satisfied by the basic functions $j$ and $x$ of [22]:

$$
\begin{gathered}
j(g):=j(i(g, 1))=j(i(1, g)), \\
x(i(1, g))=(-1)^{j(g)} x(i(g, 1)),
\end{gathered}
$$

and

$$
\beta_{V, \chi}(i(1, g))=\chi_{V}(-1)^{j(g)} \beta_{V, \chi}(i(g, 1))
$$

On the other hand,

$$
\overline{\beta_{V, \chi}(g)}=\chi_{V}(-1)^{j(g)} \beta_{V, \chi^{-1}}(g)=\chi_{V}(-1)^{j(g)} \chi_{V}(\operatorname{det} g)^{-1} \beta_{V, \chi}(g) .
$$

Continuing our calculation, we have:

$$
\left(\iota_{V}(i(g, 1)), \overline{\beta_{V, \chi}(i(1, g))}\right)=\left(\iota_{V}(i(g, 1)), \chi(\operatorname{det} g)_{V}^{-1} \beta_{V, \chi}(i(g, 1))\right),
$$

as claimed.

The following result will also play an important role below.

Lemma 1.3. Let $P_{\mathbb{Y}}$ be the maximal parabolic subgroup of $S p\left(\mathbb{W}+\mathbb{W}_{-}\right)$which preserves $\mathbb{Y}$, and let $M_{\mathbb{Y}}$ be the Levi factor of $P_{\mathbb{Y}}$ which preserves the complete polarization

$$
\mathbb{W}+\mathbb{W}-=\mathbb{X}+\mathbb{Y}
$$

Then, for $g \in G(W), \iota_{V} \circ i \circ \Delta(g) \in M_{\mathbb{Y}}$, and

$$
\tilde{\iota}_{V, \chi}(i(\Delta(g)))=\left(\left(\begin{array}{cc}
1_{V} \otimes{ }^{t} \bar{g}^{-1} & 0 \\
0 & 1_{V} \otimes g
\end{array}\right), \chi_{V}(\operatorname{det} g)\right) \in S p\left(\mathbb{W}+\mathbb{W}_{-}\right) \times \mathbb{C}^{1} .
$$


Proof. It is clear that $\iota_{V}(i(\Delta(g)))$ preserves the complete polarization, and that it acts on $\mathbb{Y}=V \otimes \Delta(W)$ by $1_{V} \otimes g$. The element $i(\Delta(g)) \in G\left(W+W_{-}\right)$similarly preserves $Y$ and acts on $Y$ by $g$. Thus $j(i(\Delta(g)))=0$ and $x(g)=\operatorname{det}(g)$. Thus

$$
\beta_{V, \chi}(i(\Delta(g)))=\chi_{V}(\operatorname{det}(g)),
$$

as claimed.

Finally, we need to understand the effect of the involutions considered by MoeglinVigneras-Waldspurger [29] (the MVW involutions). Let $g_{0} \in G L_{F}(W)$ and $h_{0} \in$ $G L_{F}(V)$ be $\tau$-linear automorphisms such that

$$
\left\langle w_{1} g_{0}, w_{2} g_{0}\right\rangle=\left\langle w_{2}, w_{1}\right\rangle, \quad \text { for all } w_{1} \text { and } w_{2} \in W
$$

and

$$
\left(h_{0} v_{1}, h_{0} v_{2}\right)=\left(v_{2}, v_{1}\right), \quad \text { for all } v_{1} \text { and } v_{2} \in V \text {. }
$$

Note that, since $g_{0}$ and $h_{0}$ are both $\tau$-linear, $h_{0} \otimes g_{0} \in G L_{F}(\mathbb{W})$ is well defined, and that

$$
\left\langle\left\langle\left(h_{0} \otimes g_{0}\right) z_{1},\left(h_{0} \otimes g_{0}\right) z_{2}\right\rangle\right\rangle=\left\langle\left\langle z_{2}, z_{1}\right\rangle\right\rangle=-\left\langle\left\langle z_{1}, z_{2}\right\rangle\right\rangle
$$

for all $z_{1}, z_{2} \in \mathbb{W}$, so that $h_{0} \otimes g_{0}$ is an element of $G S p(\mathbb{W})$ with scale -1 . Let $A: S p(\mathbb{W}) \longrightarrow S p(\mathbb{W})$ be the outer automorphism $A=A d\left(h_{0} \otimes g_{0}\right)$. Note that there is a unique lift $\tilde{A}$ of $A$ to $M p(\mathbb{W})$ which is anti-linear on $\mathbb{C}^{1}$.

Lemma 1.4. The following diagram commutes:

$$
\begin{array}{ccc}
G(W) & \stackrel{\tilde{\iota}_{V, \chi}}{\longrightarrow} & M p(\mathbb{W}) \\
A d\left(g_{0}\right) \downarrow & \downarrow \tilde{A} \\
G(W) & \stackrel{\tilde{\iota}_{V, \chi}}{\longrightarrow} & M p(\mathbb{W})
\end{array}
$$

Proof. We begin by doubling the diagram of the Lemma, writing $U=W+W_{-}$ and $\mathbb{U}=\mathbb{W}+\mathbb{W}_{-}$,

$$
\begin{array}{ccccc}
G(W) \times G(W) & \stackrel{i}{\rightarrow} & G(U) & \longrightarrow & M p(\mathbb{U}) \\
A_{0} \times A_{0} \downarrow & & B_{0} \downarrow & & \tilde{B} \downarrow \\
G(W) \times G(W) & \stackrel{i}{\rightarrow} & G(U) & \longrightarrow & M p(\mathbb{U}) .
\end{array}
$$

Here $B_{0}=A d\left(g_{0} \times g_{0}\right)$ and $B=A d\left(h_{0} \otimes\left(g_{0} \times g_{0}\right)\right)$. It suffices to prove that the right hand square in this diagram commutes. Moreover, since the anti-linear maps $\gamma: U \longrightarrow U$ such that $\left\langle u_{1} \gamma, u_{2} \gamma\right\rangle=\left\langle u_{2}, u_{1}\right\rangle$ form a single $G(U)$ coset, it suffices to check the commutativity for a single such $\gamma$. If $e_{1}, \ldots, e_{n}, e_{1}^{\prime}, \ldots, e_{n}^{\prime}$ is a standard 'symplectic' basis for $U$, let $\gamma$ be the $E$ anti-linear isomorphism such that $e_{i} \gamma=e_{i}$ and $e_{i}^{\prime} \gamma=-e_{i}^{\prime}$ for all $i$. Let $Y$ be the $E$ span of the $e_{i}^{\prime \prime}$ s, $\mathbb{Y}=V \otimes Y$, and let

$$
M p(\mathbb{U}) \simeq S p(\mathbb{U}) \times \mathbb{C}^{1}
$$


be the asociated isomorphism. Let $C_{0}=A d(\gamma)$ on $G(U)$, and let $C=A d\left(h_{0} \otimes \gamma\right)$ on $S p(\mathbb{U})$. Then, since $h_{0} \otimes \gamma$ preserves $\mathbb{Y}$, the lift $\tilde{C}$ of $C$ to $M p(\mathbb{U})$ is given by

$$
(g, \epsilon) \mapsto(C \cdot g, \bar{\epsilon}),
$$

while the map $\tilde{\iota}_{V, \chi}$ is given by $g \mapsto\left(\iota_{V}(g), \beta_{V, \chi}(g)\right)$. Now, if we let $c: U \longrightarrow U$ be the $E$-linear similitude whose action on the standard basis is the same as that of $\gamma$, then, for $g \in G(U)$,

$$
C_{0} \cdot g=A d(c) \cdot \bar{g}
$$

Now

$$
\begin{aligned}
x(\bar{g}) & =\overline{x(g)}, \\
x(A d(c) \cdot g) & =(-1)^{j(g)} x(g),
\end{aligned}
$$

and $j(\bar{g})=j(A d(c) \cdot g)=j(g)$. Thus

$$
\begin{aligned}
\beta_{V, \chi}\left(C_{0} \cdot g\right) & =\chi(x(A d(c) \cdot \bar{g})) \gamma_{F}(\eta \circ R V)^{-j(g)} \\
& =\chi(-1)^{j(g)} \chi(\overline{x(g)}) \gamma_{F}(\eta \circ R V)^{-j(g)} \\
& =\chi(-1)^{j(g)} \chi(x(g))^{-1} \gamma_{F}(\eta \circ R V)^{-j(g) .}
\end{aligned}
$$

Since

$$
\gamma_{F}(\eta \circ R V)=(\Delta, \operatorname{det} V)_{F} \gamma_{F}(-\Delta, \eta)^{m} \gamma_{F}(-1, \eta)^{-m},
$$

we find that

$$
\overline{\gamma_{F}(\eta \circ R V)}=(-1, \Delta)_{F}^{m} \gamma_{F}(\eta \circ R V) .
$$

Thus, since $\chi(-1)^{j(g)}=(-1, \Delta)_{F}^{m j(g)}$, we have

$$
\beta_{V, \chi}\left(C_{0} \cdot g\right)=\overline{\beta_{V, \chi}(g)} .
$$

This proves the required commutativity.

So far we have only discussed the splitting $\tilde{\iota}_{V, \chi}$ over $G(W)$, where $W$ is the skew-Hermitian space in the pair $(V, W)$. To obtain a splitting over $G(V)$, we use the following construction. Recall that we have fixed $\delta$ with $\bar{\delta}=-\delta$. Let $W^{\prime}=W$ with Hermitian form

$$
\left(w_{1}^{\prime}, w_{2}^{\prime}\right)^{\prime}=\delta^{-1}\left\langle w_{2}, w_{1}\right\rangle,
$$

and let $V^{\prime}=V$ with skew-Hermitian form

$$
\left\langle v_{1}^{\prime}, v_{2}^{\prime}\right\rangle=\delta\left(v_{2}, v_{1}\right)
$$

Here, for $w \in W$ (resp. $v \in V$ ), we let $w^{\prime}$ (resp. $v^{\prime}$ ) denote the corresponding element of $W^{\prime}$ (resp. $\left.V^{\prime}\right)$. Applying the previous constructions to the pair $\left(W^{\prime}, V^{\prime}\right)$, we obtain a splitting

$$
\tilde{\iota}_{W^{\prime}, \chi^{\prime}}: G(V)=G\left(V^{\prime}\right) \longrightarrow M p\left(\mathbb{V}^{\prime}\right),
$$


where $\mathbb{V}^{\prime}=W^{\prime} \otimes_{E} V^{\prime}$, and where $\chi^{\prime}$ is a character of $E^{\times}$whose restriction to $F^{\times}$ is $\epsilon_{E / F}^{n}$, for $n=\operatorname{dim}_{E} W$. Note that the map $v \otimes w \mapsto w^{\prime} \otimes v^{\prime}$ defines an isometry $\mathbb{W} \longrightarrow \mathbb{V}^{\prime}$, since

$$
\begin{aligned}
\left(w_{1}^{\prime}, w_{2}^{\prime}\right)^{\prime} \overline{\left\langle v_{1}^{\prime}, v_{2}^{\prime}\right\rangle^{\prime}} & =\delta^{-1}\left\langle w_{2}, w_{1}\right\rangle \overline{\delta\left(v_{2}, v_{1}\right)} \\
& =\left(v_{1}, v_{2}\right) \overline{\left\langle w_{1}, w_{2}\right\rangle} .
\end{aligned}
$$

Thus we have an isomorphism $\alpha: M p\left(\mathbb{V}^{\prime}\right) \longrightarrow M p(\mathbb{W})$ (restricting to the identity map on $\mathbb{C}^{1}$ ), and we define

$$
\tilde{\iota}_{W, \chi^{\prime}}=\alpha \circ \tilde{\iota}_{W^{\prime}, \chi^{\prime}}: G(V) \longrightarrow M p(\mathbb{W}) .
$$

The various results of this section carry over to $\tilde{\iota}_{W, \chi^{\prime}}$.

\section{§2. WEIL REPRESENTATIONS}

We may now define the Weil representations of the dual pairs $(G(V), G(W))$, $\left(G\left(W+W_{-}\right), G(V)\right)$, etc., and we determine their relations via the doubling seesaw.

For our fixed additive character $\psi$ of $F$, let $\left(\omega_{\psi}, S\right)$ be the Weil representation of $M p(\mathbb{W})$ or of $M p\left(\mathbb{W}+\mathbb{W}_{-}\right)$. In the latter case, we consider the standard Schrödinger model associated to the complete polarization $\mathbb{X}+\mathbb{Y}$, and so we take $S=S(\mathbb{X})=$ $S\left(V^{n}\right)$. Here the identification of $\mathbb{X}=V \otimes X$ is obtained from a standard $E$-basis for $W+W_{-}$(cf. [22], sections 4 and 5). Note that, in both cases, the central $\mathbb{C}^{1}$ acts by its holomorphic identity character, i.e.,

$$
\omega_{\psi}(z)=\text { multiplication by } z \text {. }
$$

We fix a pair of characters $\chi=\left(\chi_{n}, \chi_{m}\right)$ of $E^{\times}$with

$$
\left.\chi_{m}\right|_{F^{\times}}=\epsilon_{E / F}^{m} \quad \text { and }\left.\quad \chi_{n}\right|_{F^{\times}}=\epsilon_{E / F}^{n},
$$

where $m=\operatorname{dim}_{E} V$ and $n=\operatorname{dim}_{E} W$. These characters define splitting homomorphisms

$$
\begin{aligned}
& \tilde{\iota}_{V, \chi_{m}}: G(W) \longrightarrow M p(\mathbb{W}), \\
& \tilde{\iota}_{V, \chi_{m}}: G\left(W+W_{-}\right) \longrightarrow M p\left(\mathbb{W}+\mathbb{W}_{-}\right),
\end{aligned}
$$

and

$$
\begin{aligned}
& \tilde{\iota}_{W, \chi_{n}}: G(V) \longrightarrow M p(\mathbb{W}), \\
& \tilde{\iota}_{W, \chi_{n}}: G\left(V+V_{-}\right) \longrightarrow M p\left(\mathbb{W}+\mathbb{W}_{-}\right) .
\end{aligned}
$$

We will often write $\chi$ in place of $\chi_{m}$ or $\chi_{n}$, since the choice of the relevant element of the pair should be clear from context. Using these, we define representations

$$
\omega_{V, \chi}=\omega_{\psi} \circ \tilde{\iota}_{V, \chi}
$$

of $G(W)$ or of $G\left(W+W_{-}\right)$and

$$
\omega_{W, \chi}=\omega_{\psi} \circ \tilde{\iota}_{W, \chi}
$$

of $G(V)$ or of $G\left(V+V_{-}\right)$. Hopefully the relevant group will also be clear from the context. 
The following facts are well known:

Lemma 2.1. (i) ([40]) Under the homomorphism

$$
\tilde{i}: M p(\mathbb{W}) \times M p(\mathbb{W}) \longrightarrow M p(\mathbb{W}+\mathbb{W}-),
$$

of (1.17),

$$
\omega_{\psi} \circ \tilde{i}=\omega_{\psi} \otimes \omega_{\psi}^{\vee},
$$

where $\omega_{\psi}^{\vee}$ is the contragredient of $\omega_{\psi}$.

(ii) ([29]) If $\pi$ is an irreducible admissible representation of $G(W)$, then

$$
\pi \circ \operatorname{Ad}\left(g_{0}\right) \simeq \pi^{\vee},
$$

where $A d\left(g_{0}\right)$ is as in (1.32). Similarly, if $\sigma$ is an irreducible admissible representation of $G(V)$, then

$$
\sigma \circ A d\left(h_{0}\right) \simeq \sigma^{\vee}
$$

where $h_{0}$ is as in (1.33).

Remark. Here (ii) is a deep result of Moeglin-Vigneras-Waldspurger, in spite of the label here. Also, in (i), the anti-linearity (1.18) of $\tilde{i}$ on the second central $\mathbb{C}^{1}$ is essential.

The results of section 1 now yield the following information on pullbacks:

Proposition 2.2. (i) As representations of $G(W) \times G(W)$,

$$
\omega_{V, \chi} \circ i \simeq \omega_{V, \chi} \otimes\left(\chi_{m} \cdot\left(\omega_{V, \chi}\right)^{\vee}\right),
$$

and, as representations of $G(V) \times G(V)$,

$$
\omega_{W, \chi} \circ i \simeq \omega_{W, \chi} \otimes\left(\chi_{n} \cdot\left(\omega_{W, \chi}\right)^{\vee}\right) .
$$

(ii) The representation $\omega_{V, \chi} \circ i \circ \Delta$ of $G(W)$ is isomorphic to the twist by $\chi_{m}$ of the linear action of $G(W)$ on $S\left(V^{n}\right)$, i.e., for $\varphi \in S\left(V^{n}\right)$ and $x \in V^{n}$,

$$
\omega_{V, \chi}(i(g, g)) \varphi(x)=\chi_{m}(\operatorname{det} g) \cdot \varphi\left(x^{t} \bar{g}^{-1}\right) .
$$

Similarly, the representation $\omega_{W, \chi} \circ i \circ \Delta$ of $G(V)$ is isomorphic to the twist by $\chi_{n}$ of the linear action of $G(V)$ on $S\left(V^{n}\right)$, i.e., for $\varphi \in S\left(V^{n}\right)$ and $x \in V^{n}$,

$$
\omega_{W, \chi}(i(h, h)) \varphi(x)=\chi_{n}(\operatorname{det} h) \cdot \varphi\left(h^{-1} x\right) .
$$

(iii) For the $M V W$ involutions,

$$
\omega_{V, \chi} \circ \operatorname{Ad}\left(g_{0}\right) \simeq\left(\omega_{V, \chi}\right)^{\vee} \text { and } \omega_{W, \chi} \circ \operatorname{Ad}\left(h_{0}\right) \simeq\left(\omega_{W, \chi}\right)^{\vee} .
$$

Proof. We have, for example,

$$
\begin{aligned}
\omega_{V, \chi} \circ i & =\omega_{\psi} \circ \tilde{\iota}_{V, \chi} \circ i \\
& =\omega_{\psi} \circ \tilde{i} \circ\left(\tilde{\iota}_{V, \chi} \times \tilde{\iota}_{V, \chi,-}\right) \\
& =\omega_{\psi} \circ \tilde{i} \circ\left(\tilde{\iota}_{V, \chi} \times \chi_{m}^{-1} \cdot \tilde{\iota}_{V, \chi}\right) \\
& =\omega_{V, \chi} \otimes\left(\chi_{m} \cdot\left(\omega_{V, \chi}\right)^{\vee}\right),
\end{aligned}
$$

where we note that the anti-linear action of the central $\mathbb{C}^{1}$ for $\omega_{\psi}^{\vee}$ inverts the $\chi_{m}^{-1}$ in the last step. 
To prove (iii), we consider the diagram of Lemma 1.4 and its analogue for $\tilde{\iota}_{W, \chi}$. Then

$$
\begin{aligned}
\omega_{V, \chi} \circ \operatorname{Ad}\left(g_{0}\right) & =\omega_{\psi} \circ \tilde{\iota}_{V, \chi} \circ \operatorname{Ad}\left(g_{0}\right) \\
& =\omega_{\psi} \circ \tilde{A} \circ \tilde{\iota}_{V, \chi} \\
& =\left(\omega_{\psi}\right)^{\vee} \circ \tilde{\iota}_{V, \chi} \\
& =\left(\omega_{V, \chi}\right)^{\vee} .
\end{aligned}
$$

\section{§3. The THeta CORRESPONDENCE}

For convenience, we now write $\left(\omega_{V, W, \chi}, S_{V, W, \chi}\right)$ for the Weil representation $\omega_{V, \chi} \otimes$ $\omega_{W, \chi}$ on the space $S_{V, W, \chi}$. Note that the Weil representations $\omega_{V, W+W_{-}, \chi}$ of $\left(G(V), G\left(W+W_{-}\right)\right)$and $\omega_{V+V_{-}, W, \chi}$ of $\left(G\left(V+V_{-}\right), G(W)\right)$ are both realized on the space $S\left(V^{n}\right)$.

If $\pi$ is an irreducible admissible representation of $G(W)$, let $S_{V, W, \chi}(\pi)$ be the maximal quotient of $S_{V, W, \chi}$ on which $G(W)$ acts as a multiple of $\pi$. Write

$$
S_{V, W, \chi}(\pi) \simeq \pi \otimes \Theta_{\chi}(\pi, V),
$$

where $\Theta_{\chi}(\pi, V)$ is a representation of $G(V)$. We set $\Theta_{\chi}(\pi, V)=0$ if $\pi$ does not occur as a quotient of $S_{V, W, \chi}$. The Howe duality principle asserts that

(i) $\Theta_{\chi}(\pi, V)$ is a finitely generated admissible representation of $G(V)$.

(ii) $\Theta_{\chi}(\pi, V)$ has a unique proper maximal $G(V)$-invariant subspace and a unique irreducible quotient $\theta_{\chi}(\pi, V)$.

(iii) The correspondence $\pi \mapsto \theta_{\chi}(\pi, V)$ defines a bijection between the subsets of $\operatorname{Irr}(G(W))$ and $\operatorname{Irr}(G(V))$ consisting of irreducible admissible representations which occur as quotients of $S_{V, W, \chi}$.

This is known to hold if the residue characteristic of $F$ is not 2 [50]. Here we only assume a consequence of (i):

(i) ${ }^{\prime}$ As a representation of $G(V), \Theta_{\chi}(\pi, V)$ admits an irreducible quotient $\sigma$.

In fact, when $\pi$ is supercuspidal, we will prove (Proposition 5.4, below) that (i) ${ }^{\prime}$ is always satisfied.

Now consider the dual pair $\left(G\left(W+W_{-}\right), G(V)\right)$, and note that, by Proposition 2.2, for the Weil representation $\omega_{V, W+W_{-}, \chi}$ described there, the group $G(V)$ acts via the twist by $\chi_{n}$ of its linear action in $S\left(V^{n}\right)$. Let

$$
R_{n}\left(V, \chi_{m}\right)=S\left(V^{n}\right)\left(\chi_{n}\right)=\Theta_{\chi}\left(\chi_{n}, W+W_{-}\right)
$$

be the maximal quotient on which $G(V)$ acts by the character $\chi_{n}$. Alternatively, $R_{n}\left(V, \chi_{m}\right)$ is the maximal quotient of $S\left(V^{n}\right)$, with the linear action of $G(V)$, on which $G(V)$ acts trivially. Note that $R_{n}\left(V, \chi_{m}\right)$ is known [25] to be an admissible representation of $G\left(W+W_{-}\right)$of finite length and with a unique irreducible quotient in all cases, i.e., for any residue characteristic. Observe that it is the character $\chi_{m}$, with $\left.\chi_{m}\right|_{F^{\times}}=\epsilon_{E / F}^{m}, m=\operatorname{dim}_{E} V$, which determines the action of $G\left(W+W_{-}\right)$in this space. We will usually abuse notation and write $R_{n}(V, \chi)$ for this representation. 
A key result is then the following:

Proposition 3.1. Assume (i)'. Then, for any irreducible admissible representation $\pi$ of $G(W)$,

$$
\Theta_{\chi}(\pi, V) \neq 0 \Longleftrightarrow \operatorname{Hom}_{G(W) \times G(W)}\left(R_{n}(V, \chi), \pi \otimes\left(\chi \cdot \pi^{\vee}\right)\right) \neq 0 .
$$

Proof. By definition, we have

$$
\Theta_{\chi}(\pi, V) \neq 0 \Longleftrightarrow \operatorname{Hom}_{G(W)}\left(\omega_{V, W, \chi}, \pi\right) \neq 0,
$$

and so, since we assume (i) ${ }^{\prime}$,

$$
\Theta_{\chi}(\pi, V) \neq 0 \Longleftrightarrow \operatorname{Hom}_{G(W) \times G(V)}\left(\omega_{V, W, \chi}, \pi \otimes \sigma\right) \neq 0,
$$

for some irreducible admissible representation $\sigma$ of $G(V)$. If $\lambda: \omega_{V, W, \chi} \longrightarrow \pi \otimes \sigma$ is a non-zero intertwining map, then, applying the MVW involution to both factors, we have a non-zero $\lambda^{0}: \omega_{V, W, \chi}^{\vee} \longrightarrow \pi^{\vee} \otimes \sigma^{\vee}$. Then, writing $G=G(W)$ and $H=G(V)$ and assuming (i)' in the first step, we have

$$
\begin{aligned}
& \operatorname{Hom}_{G}\left(\omega_{V, \chi}, \pi\right) \neq 0 \\
\Longleftrightarrow & \operatorname{Hom}_{G \times H}\left(\omega_{V, W, \chi}, \pi \otimes \sigma\right) \neq 0 \\
\Longrightarrow & \operatorname{Hom}_{G \times G \times H \times H}\left(\omega_{V, W, \chi} \otimes \omega_{V, W, \chi}^{\vee}, \pi \otimes \pi^{\vee} \otimes \sigma \otimes \sigma^{\vee}\right) \neq 0 \\
\Longrightarrow & \operatorname{Hom}_{G \times G \times H^{\Delta}}\left(\omega_{V, W, \chi} \otimes \omega_{V, W, \chi}^{\vee}, \pi \otimes \pi^{\vee} \otimes \mathbb{1}\right) \neq 0 \\
\Longleftrightarrow & H_{G \times m \times H^{\Delta}}\left(i^{*} \omega_{V, W+W-, \chi}, \pi \otimes\left(\chi \cdot \pi^{\vee}\right) \otimes \chi\right) \neq 0 \\
\Longleftrightarrow & \operatorname{Hom}_{G \times G}\left(i^{*} R_{n}(V, \chi), \pi \otimes\left(\chi \cdot \pi^{\vee}\right)\right) \neq 0 .
\end{aligned}
$$

To complete the proof, note that if any of the last three Hom's are non-zero, then

$$
\operatorname{Hom}_{G \times G}\left(\omega_{V, \chi} \otimes \omega_{V, \chi}^{\vee}, \pi \otimes \pi^{\vee}\right) \neq 0,
$$

by forgetting the action of $G(V)$. But the non-vanishing of this last space easily implies that

$$
\operatorname{Hom}_{G(W)}\left(\omega_{V, \chi}, \pi\right) \neq 0
$$

and we are done.

Remark. The argument for this proof has its origin in [36].

\section{$\S 4$. Theta Dichotomy}

Let $P_{Y}$ be the maximal parabolic subgroup of $G\left(W+W_{-}\right)$which stabilizes $Y$. The Levi factor $M_{Y}$ of $P_{Y}$ which stabilizes the complete polarization $W+W_{-}=$ $X+Y$ is isomorphic to $G L(n, E)$ via restriction to $X \simeq E^{n}$. We write $m(a) \in M_{Y}$ for the element corresponding to $a \in G L(n, E)$. For $s \in \mathbb{C}$ and for any character $\chi$ of $E^{\times}$, let $I_{n}(s, \chi)$ be the degenerate principal series consisting of smooth functions on $G\left(W+W_{-}\right)$which satisfy

$$
\Phi(n m(a) g, s)=\chi(\operatorname{det} a)|\operatorname{det} a|_{E}^{s+\frac{n}{2}} \Phi(g, s),
$$

where $n \in N_{Y}$, the unipotent radical of $P_{Y}$. 
For $V$ of dimension $m$ over $E$, as before, set $s_{0}=\frac{m-n}{2}$, and assume that

$$
\left.\chi\right|_{F \times}=\epsilon_{E / F}^{m} .
$$

These conventions will be fixed throughout this section. Then the map

$$
\begin{aligned}
S\left(V^{n}\right) & \longrightarrow I_{n}\left(s_{0}, \chi\right), \\
\varphi & \mapsto \omega_{V, \chi}(g) \varphi(0),
\end{aligned}
$$

factors through $R_{n}(V, \chi)$, and, by [29], induces an injection

$$
R_{n}(V, \chi) \hookrightarrow I_{n}\left(s_{0}, \chi\right) .
$$

Recall that the isometry class of the Hermitian space $V$ of dimension $m$ over $E$ is determined by the sign

$$
\epsilon(V)=\epsilon_{E / F}\left((-1)^{\frac{m(m-1)}{2}} \operatorname{det} V\right),
$$

where $\operatorname{det} V=\operatorname{det}\left(\left(x_{i}, x_{j}\right)\right) \in F^{\times} / N E^{\times}$, for any $E$-basis $x_{1}, \ldots, x_{m}$ for $V$. We will write $V_{m}^{+}$and $V_{m}^{-}$for the corresponding pair of Hermitian spaces. The following result is proved in [25].

Proposition 4.1. (i) If $m \leq n$, then $R_{n}(V, \chi)$ is irreducible, and

$$
R_{n}\left(V_{m}^{+}, \chi\right) \oplus R_{n}\left(V_{m}^{-}, \chi\right)
$$

is the maximal completely reducible submodule of $I_{n}\left(s_{0}, \chi\right)$.

(ii) If $m=n$, then

$$
I_{n}(0, \chi)=R_{n}\left(V_{n}^{+}, \chi\right) \oplus R_{n}\left(V_{n}^{-}, \chi\right) .
$$

(iii) If $n<m<2 n$, then

$$
I_{n}\left(s_{0}, \chi\right)=R_{n}\left(V_{m}^{+}, \chi\right)+R_{n}\left(V_{m}^{-}, \chi\right),
$$

and $R_{n}\left(V_{m}^{+}, \chi\right) \cap R_{n}\left(V_{m}^{-}, \chi\right)$ is the unique irreducible submodule of $I_{n}\left(s_{0}, \chi\right)$. Moreover, the normalized intertwining operator

$$
M^{* *}\left(s_{0}, \chi\right): I_{n}\left(s_{0}, \chi\right) \longrightarrow I_{n}\left(-s_{0}, \chi\right)
$$

(see [25] and Lemma 6.7 (i) below) has kernel $R_{n}\left(V_{m}^{+}, \chi\right) \cap R_{n}\left(V_{m}^{-}, \chi\right)$. Also

$$
M^{* *}\left(s_{0}, \chi\right)\left(R_{n}\left(V_{m}^{ \pm}, \chi\right)\right)=R_{n}\left(V_{n-m}^{ \pm}, \chi\right)
$$

(iv) If $m=2 n$, then $V_{2 n}^{+}$is the split Hermitian space of dimension $m$, and

$$
I_{n}\left(s_{0}, \chi\right)=R_{n}\left(V_{2 n}^{+}, \chi\right) .
$$

Moreover, $R_{n}\left(V_{2 n}^{-}, \chi\right)$ is the unique irreducible submodule of $I_{n}\left(s_{0}, \chi\right)$, and the quotient $I_{n}\left(s_{0}, \chi\right) / R_{n}\left(V_{2 n}^{-}, \chi\right)$ is one dimensional, with $G\left(W+W_{-}\right)$acting via the character $\chi \circ$ det.

(v) If $m>2 n$, then $I_{n}\left(s_{0}, \chi\right)=R_{n}\left(V_{m}^{ \pm}, \chi\right)$ is irreducible.

Corollary 4.2. Let $\pi$ be an irreducible admissible representation of $G=G(W)$. For $1 \leq m \leq n$, let $s_{0}=\frac{m-n}{2}$. Then

$$
\begin{aligned}
\operatorname{dim} \operatorname{Hom}_{G \times G}\left(I_{n}\left(-s_{0}, \chi\right), \pi \otimes\left(\chi \cdot \pi^{\vee}\right)\right) & \\
\geq \operatorname{dim} \operatorname{Hom}_{G \times G}( & \left.R_{n}\left(V_{m}^{+}, \chi\right), \pi \otimes\left(\chi \cdot \pi^{\vee}\right)\right) \\
& \quad+\operatorname{dim} \operatorname{Hom}_{G \times G}\left(R_{n}\left(V_{m}^{-}, \chi\right), \pi \otimes\left(\chi \cdot \pi^{\vee}\right)\right) .
\end{aligned}
$$

Moreover, equality holds if $m=n$. 
Now we have the following variant of a result of Piatetski-Shapiro and Rallis $[32]$.

Theorem 4.3. (i) If $\pi$ is any irreducible admissible representation of $G(W)$, then, for all $s$,

$$
\operatorname{dim} \operatorname{Hom}_{G \times G}\left(I_{n}(s, \chi), \pi \otimes\left(\chi \cdot \pi^{\vee}\right)\right) \geq 1 .
$$

(ii) If $\pi$ is an irreducible supercuspidal representation of $G(W)$, or, more generally, if $\pi$ does not occur in the boundary at $s=s_{0}$, (see Definition 4.6 below), then

$$
\operatorname{dim} \operatorname{Hom}_{G \times G}\left(I_{n}\left(s_{0}, \chi\right), \pi \otimes\left(\chi \cdot \pi^{\vee}\right)\right)=1 .
$$

Combining Theorem 4.3, Corollary 4.2 and Proposition 3.1, we obtain:

Corollary 4.4 (Theta dichotomy). Suppose that $m \leq n$, and fix a character $\chi$ of $E^{\times}$such that $\left.\chi\right|_{F^{\times}}=\epsilon_{E / F^{m}}^{m}$. Let $\pi$ be an irreducible admissible supercuspidal representation of $G(W)$, or, more generally, supppose that $\pi$ is any irreducible admissible representation which does not occur in the boundary at $s=s_{0}=\frac{n-m}{2}$, (see Definition 4.6 below). Then at most one of the two theta lifts $\Theta_{\chi}\left(\pi, V_{m}^{ \pm}\right)$is non-zero. Moreover, if $m=n$ then precisely one is non-zero.

The case $n=3$ and $m=1$ or 2 of this result was proved by Gelbart, Rogawski and Soudry by a global method [10].

Corollary 4.5. If $\pi$ is any irreducible admissible representation of $G=G(W)$ and if $m \geq n$, then there exists a $V$ with $\operatorname{dim}_{E} V=m$ such that $\Theta_{\chi}(\pi, V) \neq 0$.

Proof. Since, in this case $I_{n}\left(s_{0}, \chi\right)$ is the sum (not necessarily direct) of the $R_{n}(V, \chi)^{\prime}$ 's, a non-trivial homomorphism from $I_{n}\left(s_{0}, \chi\right)$ to $\pi \otimes\left(\chi \cdot \pi^{\vee}\right)$ must have non-zero restriction to at least one such space, and Proposition 3.1 again yields the claimed result.

Note that, by 'reflection', the same results hold for supercuspidal representations of $G(V)$ and theta lifts to $G(W)$ 's with $\operatorname{dim}_{E} W=\operatorname{dim}_{E} V$.

Proof of Theorem 4.3. We postpone the proof of part (i) of the Theorem until section 6 , below.

To prove (ii), we write $G=G(W)$. The restriction of the induced representation $I_{n}(s, \chi)$ to $G \times G$ has a filtration arising from the orbit structure of $P_{Y} \backslash G\left(W+W_{-}\right)$, the space of isotropic $n$-planes in $W+W_{-}$, for the action of $G \times G$ [36], [32], [33]. The orbit of an $n$-plane $Z$ is determined by the invariant

$$
d=\operatorname{dim}(Z \cap W)=\operatorname{dim}\left(Z \cap W_{-}\right) .
$$

Note that $0 \leq d \leq r_{0}$, where $r_{0}$ is the Witt index of $W$. We write $\Omega_{d}$ for the corresponding orbit, and choose a double coset representative $\delta_{d} \in G\left(W+W_{-}\right)$for $\Omega_{d}=P \delta_{d} i(G \times G)$. The unique open orbit $\Omega_{0}$ is the orbit of $Y$, and the closure of $\Omega_{r}$ is

$$
\bar{\Omega}_{r}=\coprod_{r^{\prime} \geq r} \Omega_{r^{\prime}}
$$


Let

$$
I_{n}(s, \chi)=I_{n}^{\left(r_{0}\right)}(s, \chi) \supset I_{n}^{\left(r_{0}-1\right)}(s, \chi) \supset \cdots \supset I_{n}^{(0)}(s, \chi),
$$

be the filtration by support, and let

$$
Q_{n}^{(r)}(s, \chi)=I_{n}^{(r)}(s, \chi) / I_{n}^{(r-1)}(s, \chi) \simeq \operatorname{Ind}_{S t_{r}}^{G \times G}\left(\xi_{r, s}\right) .
$$

Here $S t_{r}$ is the stabilizer in $G \times G$ of the coset $P_{Y} \cdot \delta_{r}$ in $\Omega_{r} \simeq P_{Y} \backslash P_{Y} \delta_{r}(G \times G)$, and $\xi_{r, s}$ is the character of $S t_{r}$ defined by

$$
\xi_{r, s}(g)=\chi(\operatorname{det} a)|\operatorname{det} a|_{E}^{s+\rho_{n}}
$$

where $\delta_{r} g \delta_{r}^{-1}=n m(a) \in P_{Y}$.

Definition 4.6. We say that an irreducible admissible representation $\pi$ of $G$ does not occur in the boundary at the point $s=s_{0}$ if $\pi \otimes \chi \pi^{\vee}$ does not occur as a quotient of any $Q_{n}^{(r)}\left(s_{0}, \chi\right)$ for $r>0$.

Lemma 4.7. If $\pi$ is a supercuspidal representation of $G$, then $\pi$ does not occur in the boundary at any point $s_{0}$.

Proof. For the case of $\pi$ supercuspidal, we only need the following facts. First, for $r>0, S t_{r}$ contains the full unipotent radical $U$ of a parabolic subgroup of $G \times G$ and $\xi_{r, s} \mid U=1$. Thus

$$
\operatorname{Hom}_{G \times G}\left(Q_{n}^{r}(s, \chi), \pi \otimes\left(\chi \cdot \pi^{\vee}\right)\right)=0 .
$$

Now suppose that $\pi$ does not occur in the boundary at $s=s_{0}$, and consider a non-zero homomorphism $\lambda: I_{n}\left(s_{0}, \chi\right) \longrightarrow \pi \otimes\left(\chi \cdot \pi^{\vee}\right)$. If the restriction of $\lambda$ to $I_{n}^{(0)}\left(s_{0}, \chi\right)$, the last step in the filtration, were zero, then there would be a smallest $r$, with $r>0$, such that the restriction of $\lambda$ to $I_{n}^{(r)}\left(s_{0}, \chi\right)$ is non-zero. This restriction would then factor through the quotient $Q_{n}^{(r)}\left(s_{0}, \chi\right)$, contradicting our assumption on $\pi$. Thus, under our assumption on $\pi$, the restriction map

$$
\operatorname{Hom}_{G \times G}\left(I_{n}\left(s_{0}, \chi\right), \pi \otimes\left(\chi \cdot \pi^{\vee}\right)\right) \longrightarrow \operatorname{Hom}_{G \times G}\left(I_{n}^{(0)}\left(s_{0}, \chi\right), \pi \otimes\left(\chi \cdot \pi^{\vee}\right)\right)
$$

is injective.

For $r=0$, we take $\delta_{0}=1$ and have $S t_{0}=\Delta(G(W))$. As in Lemma 1.3,

$$
\xi_{0, s}(g)=\chi\left(\operatorname{det} \bar{g}^{-1}\right)=\chi(\operatorname{det} g) .
$$

For all $s$, the map $\Phi(s) \mapsto f_{s}$ given by

$$
f_{s}(g)=\Phi(i(g, 1), s),
$$

yields an intertwining isomorphism

$$
I_{n}^{(0)}(s, \chi) \stackrel{\sim}{\longrightarrow} S(G)(1 \otimes \chi),
$$

where $G \times G$ acts on $S(G)(1 \otimes \chi)$ by

$$
r\left(g_{1}, g_{2}\right) f(x)=\chi\left(g_{2}\right) f\left(g_{2}^{-1} x g_{1}\right) .
$$


But now, for any irreducible admissible representation $\pi$, there is a unique non-zero $G \times G$ intertwining map

$$
S(G) \longrightarrow \pi \otimes \pi^{\vee}
$$

given by sending $f \in S(G)$ to the functional on $\pi^{\vee} \otimes \pi$ :

$$
\xi^{\vee} \otimes \xi \mapsto \int_{G} f(g)\left\langle\pi^{\vee}(g) \xi^{\vee}, \xi\right\rangle d g
$$

Thus

$$
\operatorname{dim} \operatorname{Hom}_{G \times G}\left(S(G)(1 \otimes \chi), \pi \otimes\left(\chi \cdot \pi^{\vee}\right)\right)=1,
$$

and we obtain

$$
\operatorname{dim} \operatorname{Hom}_{G \times G}\left(I_{n}\left(s_{0}, \chi\right), \pi \otimes\left(\chi \cdot \pi^{\vee}\right)\right) \leq 1,
$$

as required.

\section{$\S 5$. A curious ExAmple}

In this section we will show that the dichotomy of Corollary 4.4 gives rise to certain infinite families of related supercuspidal representations!

Let $V_{r, r}$ be the split Hermitian space of dimension $2 r$ over $E$. We will refer to the family of Hermitian spaces $V+V_{r, r}, r=0,1, \ldots$, obtained by adding hyperbolic planes to a fixed space $V$ as the Witt tower, or simply tower, on $V$. Note that, since $\operatorname{det}\left(V+V_{r, r}\right)=(-1)^{r} \operatorname{det}(V)$, the sign $\epsilon\left(V_{m}\right)$ is constant as $V_{m}$ varies in a tower. Conversely, the spaces $V_{m}^{ \pm}$for a fixed sign \pm 1 vary in a tower.

Proposition 5.1. Fix a Witt tower $\left\{V_{m}^{\epsilon}\right\}, e= \pm 1$, and a character $\chi$ of $E^{\times}$ such that $\left.\chi\right|_{F^{\times}}=\epsilon_{E / F}^{m}$. Suppose that $\pi$ is an irreducible admissible supercuspidal representation of $G(W)$.

(i) (stable range [18]) If the Witt index $r$ of $V_{m}$ satisfies $r \geq n$, then $\Theta_{\chi}\left(\pi, V_{m}^{\epsilon}\right) \neq$ 0 .

(ii) (first ocurrence [21], [29]) If $m_{0}=m_{0}^{\epsilon}(\chi)$ is the smallest value of $m$ for which $\Theta_{\chi}\left(\pi, V_{m}^{\epsilon}\right) \neq 0$, then $\Theta_{\chi}\left(\pi, V_{m_{0}}^{\epsilon}\right)$ is an irreducible supercuspidal representation of $G\left(V_{m_{0}}^{\epsilon}\right)$.

(iii) (persistence) If $m \geq m_{0}$, then $\Theta_{\chi}\left(\pi, V_{m}^{\epsilon}\right) \neq 0$.

Remark. One should be careful to note that (ii) does not apply when $\pi$ is not supercuspidal, i.e., the first occurence need not be supercuspidal in general.

Proof of (iii). First observe the following useful fact.

Lemma 5.2. Suppose that $V_{1}$ and $V_{2}$ are Hermitian spaces of dimensions $m_{1}$ and $m_{2}$, and let $V=V_{1}+V_{2}, \mathbb{W}_{i}=V_{i} \otimes W$, and $\mathbb{W}=\mathbb{W}_{1}+\mathbb{W}_{2}$. Fix characters $\chi_{1}$ and $\chi_{2}$ of $E^{\times}$with restrictions $\epsilon_{E / F}^{m_{i}}$ to $F^{\times}$and let $\chi=\chi_{1} \cdot \chi_{2}$. Then the diagram

$$
\begin{array}{ccc}
G(W) & \stackrel{\tilde{\iota}_{V, \chi}}{\longrightarrow} & M p(\mathbb{W}) \\
\Delta \downarrow & \uparrow i \\
G(W) \times G(W) & \stackrel{\tilde{\iota}_{V_{1}, \chi_{1}} \times \tilde{\iota}_{V_{2}, \chi_{2}}}{\longrightarrow} & M p\left(\mathbb{W}_{1}\right) \times M p\left(\mathbb{W}_{2}\right)
\end{array}
$$

commutes, where the map $i$ is holomorphic on both central $\mathbb{C}^{1}$ 's. 
Proof. If we write $U=W+W_{-}, \mathbb{U}_{i}=V_{i} \otimes U$, etc., then it suffices to prove the result for the analogous diagram for $G(U)$. But it is easy to check that

$$
\beta_{V, \chi}(g)=\beta_{V_{1}, \chi_{1}}(g) \cdot \beta_{V_{2}, \chi_{2}}(g),
$$

and the Lemma follows. have

Applying Lemma 5.2 to the space $V_{m}^{\epsilon}=V_{m_{0}}^{\epsilon}+V_{r, r}$, with $\chi_{1}=\chi$ and $\chi_{2}=1$, we

$$
\omega_{V_{m}^{\epsilon}, W, \chi} \simeq \omega_{V_{m_{0}}^{\epsilon}, W, \chi} \otimes \omega_{V_{r, r}, W, 1} .
$$

Now, by (ii) of Proposition 2.2, the representation $\omega_{V_{r, r}, W, 1}$ can be realized in the space $S\left(W^{r}\right)$ with the group $G(W)$ acting linearly. Then, if

$$
\lambda: S_{V_{m_{0}}^{\epsilon}, W, \chi} \longrightarrow \pi
$$

is a non-zero $G(W)$-intertwining map, then

$$
\lambda \otimes \delta_{0}: S_{V_{m_{0}}, W, \chi} \otimes S\left(W^{r}\right) \longrightarrow \pi
$$

is a non-zero $G(W)$-intertwining map, where $\delta_{0}$ is the delta distribution at the origin on $S\left(W^{r}\right)$.

The combination of these three principles with the dichotomy of Corollary 4.4 gives rise to a curious phenomenon. Recall that we have fixed $\delta \in E^{\times}$with $\delta^{2}=\Delta$. We let $W_{n}^{ \pm}$be the skew-Hermitian space which becomes isomorphic to $V_{n}^{ \pm}$(in the notation introduced in section 4$)$ when we scale the skew-Hermitian form by $\delta^{-1}$. We then obtain four towers $\left\{V_{n}^{+}\right\},\left\{V_{n}^{-}\right\},\left\{W_{n}^{+}\right\}$and $\left\{W_{n}^{-}\right\}$as $n$ runs over positive integers of a fixed parity. Fix $\chi$ of the same 'parity' as $n$, i.e., which satisfies (4.4).

Let $\pi_{0}$ be an irreducible supercuspidal representation of $G\left(W_{0}\right)$, where $W_{0}=$ $W_{n_{0}}^{\epsilon_{0}}$. By dichotomy, there is a unique sign $\epsilon_{1}$ such that

$$
\Theta_{\chi}\left(\pi_{0}, V_{n_{0}}^{\epsilon_{1}}\right)=0,
$$

so that the first occurence of $\pi_{0}$ in the $V_{n}^{\epsilon_{1}}$ tower occurs for some integer $n_{1}>n_{0}$, i.e., for $V_{1}=V_{n_{1}}^{\epsilon_{1}}$,

$$
\pi_{1}=\Theta_{\chi}\left(\pi_{0}, V_{n_{1}}^{\epsilon_{1}}\right)
$$

is an irreducible supercuspidal representation of $G\left(V_{1}\right)$. By applying the same argument to $\pi_{1}$, we obtain a supercuspidal $\pi_{2}$ for some $G\left(W_{2}\right)$, where $W_{2}=W_{n_{2}}^{\epsilon_{2}}$ with $n_{2}>n_{1}$. Note, however, that since $\pi_{1}$ is a theta lift of $\pi_{0}$, we must have $\epsilon_{2}=$ $-\epsilon_{0}$. Proceeding again, we obtain a supercuspidal $\pi_{3}$ of $G\left(V_{3}\right)$, where $V_{3}=V_{n_{3}}^{\epsilon_{3}}$, and $n_{3}>n_{2}, \epsilon_{3}=-\epsilon_{1}$. This game can be continued indefinitely, and we obtain:

Proposition 5.3. Suppose that $\pi$ is an irreducible supercuspidal representation of $G(W)$, where $W=W_{n_{0}}^{\epsilon_{0}}$. Fix a character $\chi$ of $E^{\times}$with $\left.\chi\right|_{F^{\times}}=\epsilon_{E / F}^{m_{0}}$. Then there exist a sequence of integers $n_{0}<n_{1}<\ldots<n_{i}<\ldots$ and signs $\epsilon_{0}, \epsilon_{1}, \ldots$ with $\epsilon_{i}=-\epsilon_{i+2}$ and a sequence of irreducible supercuspidal representations $\pi_{i}$ of unitary groups $G_{i}$, where

$$
G_{i}=\left\{\begin{array}{l}
G\left(W_{n_{i}}^{\epsilon_{i}}\right) \quad \text { if } i \text { is even, } \\
G\left(V_{n_{i}}^{\epsilon_{i}}\right) \quad \text { if } i \text { is odd },
\end{array}\right.
$$


such that

$$
\Theta_{\chi}\left(\pi_{i}, G_{i+1}\right)=\pi_{i+1},
$$

with the obvious slight abuse of notation.

A conjectural explanation of this result from the point of view of Langlands functoriality will be given in section 7 below. In particular, Speculation 7.7 gives a conjectural description of the sequence of dimensions $n_{0}<n_{1}<\ldots$ and Lparameters which arise.

We conclude this section with a proof that the assumption (i) ${ }^{\prime}$ at the beginning of section 3 always holds for supercuspidal representations.

Proposition 5.4. Suppose that $\pi$ is a supercuspidal representation of $G(W)$. Then, as a representation of $G(V)$, the space $\Theta_{\chi}(\pi, V)$ is either zero or admits a non-zero irreducible quotient.

Proof. We consider the tower containing the space $V$, and write $V_{r}=V_{0}+V_{r, r}$ with $V_{0}$ anisotropic and $V_{r, r}$ split. For convenience we write $S_{r}$ for $S_{V_{r}, W, \chi}, \Theta_{r}(\pi)$ for $\Theta_{\chi}\left(\pi, V_{r}\right)$, and $H_{r}$ for $G\left(V_{r}\right)$.

Let $r_{0}$ be the smallest value of $r$ for which $\Theta_{r}(\pi)$ is non-zero. Then, by the calculations of [21], as extended to the unitary case in [29], all of the Jacquet functors of $\Theta_{r_{0}}(\pi)$ relative to maximal parabolics, and hence also relative to all proper parabolics, are zero. Thus $\Theta_{r_{0}}(\pi)$ is a (not necessarily irreducible) supercuspidal representation of $H_{r_{0}}$. Any such representation has an irreducible quotient, since the quotient of $H_{r_{0}}$ by its derived group is compact. Thus we have:

$$
\lambda_{0}: S_{r_{0}} \longrightarrow S_{r_{0}}(\pi) \simeq \pi \otimes \Theta_{r_{0}}(\pi) \longrightarrow \pi \otimes \sigma,
$$

for some irreducible supercuspidal representation $\sigma$ of $H_{r_{0}}$. Denote the composite map by $\lambda_{0}$.

For any $\ell>0$, let $r=r_{0}+\ell$. Then, writing

$$
V_{r}=Z+V_{r_{0}}+Z^{\prime}
$$

where $V_{\ell, \ell}=Z+Z^{\prime}$, with $Z$ and $Z^{\prime}$ isotropic, and using the mixed polarization of $V_{r} \otimes_{E} W$ derived from $Z$, we have a mixed model $S_{r_{0}} \otimes S\left(W^{\ell}\right)$ for $S_{r}$. We view elements of this model as functions on $W^{\ell}$ valued in $S_{r_{0}}$. Let $\delta_{0}$ be the delta distribution at $0 \in W^{\ell}$. Then consider the map

$$
\begin{aligned}
S_{r} \simeq S_{r_{0}} \otimes S\left(W^{\ell}\right) & \longrightarrow(\pi \otimes \sigma), \\
\lambda_{1}: f & \mapsto \lambda_{0}\left(\delta_{0}(f)\right) .
\end{aligned}
$$

Let $L_{r} \simeq H_{r_{0}} \times G L_{\ell}(E)$ be the Levi factor in $H_{r}$ which preserves the decomposition (5.8). Then $\lambda_{1}$ is intertwining for $L_{r}$, where the $G L_{\ell}(E)$ factor acts on the right by a character $\xi$. Since $\lambda_{1}$ factors through $S_{r}(\pi)$, we obtain a commutative diagram:

$$
\begin{array}{rlcc}
S_{r_{0}} \otimes S\left(W^{\ell}\right) & \longrightarrow & (\pi \otimes \sigma) \\
\downarrow & \nearrow & \uparrow(*) \\
\pi \otimes \Theta_{r}(\pi) \simeq & S_{r}(\pi) & \longrightarrow & \pi \otimes \operatorname{Ind}_{Q_{\ell}}^{H_{r}}(\sigma \otimes \xi) .
\end{array}
$$


Here the bottom map is induced by the map

$$
\begin{aligned}
& S_{r} \longrightarrow \operatorname{Ind}_{Q_{\ell}}^{H_{r}}(\sigma \otimes \xi), \\
& f \mapsto\left(h \mapsto \lambda_{1}\left(\omega_{r}(h) f\right)\right),
\end{aligned}
$$

and the map $(*)$ is given by evaluation at $e \in H_{r}$. Since the composition of these maps, from $\pi \otimes \Theta_{r}(\pi)$ to $\pi \otimes \sigma$ is surjective, we obtain a nonzero map

$$
\Theta_{r}(\pi) \longrightarrow \operatorname{Ind}_{Q_{\ell}}^{H_{r}}(\sigma \otimes \xi)
$$

and hence $\Theta_{r}(\pi)$ must admit a non-zero quotient.

\section{$\S 6$. L AND EPSILON FACTORS}

In this section we will show that the dichotomy of Corollary 4.4 (in the case $m=n)$ is controlled by a certain local root number associated to the representation $\pi$ of $G(W)$, and that the 'early' theta lifts of $\pi$ are controlled by the poles of a certain local L-factor.

More precisely, the doubling method of Piatetski-Shapiro and Rallis yields a family of zeta integrals which produce, by more or less standard procedures, certain $\mathrm{L}$ and $\epsilon$-factors attached to $\pi$. If $G(W)$ is the split group and $\pi$ is an unramified principal series, and if $\chi$ is unramified, then the base change $B C(\pi)$ to an unramified principal series representation of $G L(n, E)$ is well defined, and the local zeta integrals yield $L(s, B C(\pi) \otimes \chi)$ and $\epsilon\left(s, B C(\pi) \otimes \chi, \psi_{E}\right)$, the standard $\mathrm{L}$ and $\epsilon$-factors for $B C(\pi) \otimes \chi$. The case of the L-factor was discussed by Li [28]. For general representations $\pi$, we will write $L(s, \pi, \chi)$ and $\epsilon(s, \pi, \chi, \psi)$ for the factors which arise by the doubling method. The expected relation between these factors and those defined by other means is described in the comparison conjecture $(0.18)$ and (0.19) of the introduction.

Our first main result is then the following local root number characterization of dichotomy.

Theorem 6.1 (epsilon dichotomy). Let $\pi$ be an irreducible admissible supercuspidal representation of $G(W)$, or, more generally, supppose that $\pi$ is any irreducible admissible representation which does not occur in the boundary at $s=0$ (see Definition 4.6). For $n=\operatorname{dim}_{E} W$, fix a character $\chi$ of $E^{\times}$such that $\left.\chi\right|_{F^{\times}}=\epsilon_{E / F}^{n}$.

(i) Then

$$
\epsilon\left(\frac{1}{2}, \pi, \chi, \psi\right)= \pm 1 \text {. }
$$

(ii) Let $V$ be a Hermitian space with $\operatorname{dim}_{E} V=\operatorname{dim}_{E} W=n$. Then

$$
\Theta_{\chi}\left(\pi^{\vee}, V\right) \neq 0 \Longleftrightarrow \epsilon\left(\frac{1}{2}, \pi, \chi, \psi\right)=\epsilon_{E / F}(-2)^{n} \cdot \epsilon_{E / F}(\operatorname{det} V) .
$$

Our second result links the poles of the doubling L-factor for a supercuspidal representation $\pi$ to the existence of early theta lifts. First recall that, if we fix a choice of a uniformizing parameter $\varpi_{E}$ of $E$, and let $q_{E}=\left|\varpi_{E}\right|_{E}^{-1}$, then the pair $(s, \chi)$ is called normalized if

$$
\chi\left(\varpi_{E} \cdot \overline{\varpi_{E}}\right)=1
$$

and

$$
\operatorname{Im}(\mathrm{s}) \in\left[0, \frac{\pi}{\log \left(q_{E}\right)}\right) .
$$

Also recall that $\check{\chi}(x)=\chi(\bar{x})^{-1}$, and that $\chi=\check{\chi}$ if and only if $\left.\chi\right|_{F^{\times}}=\epsilon_{E / F}$ or 1 . 
Theorem 6.2. For a supercuspidal representation $\pi$ of $G(W)$, and a unitary character $\chi$ of $E^{\times}$, let $L(s, \pi, \chi)$ be the L-factor defined by doubling. Assume that the pair $(s, \chi)$ is normalized.

(i) If $\chi \neq \check{\chi}$, then $L(s, \pi, \chi)=1$.

(ii) If $\chi=\check{\chi}$, then the poles of $L(s, \pi, \chi)$ are at most simple and lie in the set

$$
X_{n}(\chi)=\left\{\frac{m-n+1}{2} \mid 0 \leq m \leq n-1, \chi^{o}=\epsilon_{E / F}^{m}\right\},
$$

where $\chi^{o}$ denotes the restriction of $\chi$ to $F^{\times}$. Note that this set is precisely the set of poles of $b_{n}(s, \chi)$. Moreover, a pole occurs at $s_{0}=\frac{m-n+1}{2} \in X_{n}(\chi)$ if and only if there is a space $V$ with $\operatorname{dim}_{E} V=m$ such that

$$
\Theta_{\chi}\left(\pi^{\vee}, V\right) \neq 0 .
$$

Note that, if we consider theta lifts for a Witt tower, as in section 5 , then by Corollary 4.5, any $\pi$ will always have a non-trivial theta lift to one of the two possible towers provided $\operatorname{dim}_{E} V=m \geq n$. If $\pi$ is supercuspidal, then $\pi$ has a theta lift to some smaller $G(V)$, i.e., to one with $\operatorname{dim}_{E} V<n$ if and only if $L(s, \pi, \chi) \neq 1$ for some $\chi$. Moreover, by 'persistence' ( (iii) of Proposition 5.1), the degree of this Euler factor will be $-\left[\frac{m-n}{2}\right]$, where $m$ is the dimension of the smallest $V$ for which such an 'early' theta lift occurs. The compatibility of this picture with functoriality will be discussed in section 7 below.

Before proving these results, we must review the doubling method definition of $\mathrm{L}$ and $\epsilon$-factors [32], [33]. We do this in some detail for two reasons. First, Piatetski-Shapiro and Rallis introduced in [33] a certain refinement (the use of "good sections") of the method of [32]. Second, the normalization of the intertwining operator in [32] depends on an auxillary choice, and the $\epsilon$-factor depends on this choice in a way which is not determined. As a result, the actual root number remains ambiguous! In our discussion we will give a precise definition of the $\epsilon$-factor, and it is this definition which is used in Theorem 6.1.

In the case $n=1$, Theorem 6.1 was already obtained by Rogawski [41]. In fact, Rogawski states the result in terms of the base change $\epsilon$-factor, and relates this factor to the earlier result of Moen [30] on the $(U(1), U(1))$ dual pair. We will give a precise description of the relation between the base change $\epsilon$ and the doubling $\epsilon$ in section 8 below, in the case $n=1$. Thus, Theorem 6.1 together with the computations of section 8 will yield a purely local proof of Rogawski's result. In the case $n=2$, Theorem 6.1 (for similitude groups) is due to Harris [15]. Of course, the original result of this sort is that of Tunnell [47] and Waldspurger [49], and, indeed their results are used by Harris in the case of $G U(2)$.

For the moment, we allow $\chi$ to be an arbitrary character of $E^{\times}$.

We begin with the normalization of the intertwining operator; for more details the reader should consult section 3 of [25]. For a section $\Phi(s) \in I_{n}(s, \chi)$ and for $\operatorname{Re}(s)>\rho_{n}=\frac{n}{2}$, the intertwining operator

$$
M(s, \chi): I_{n}(s, \chi) \longrightarrow I_{n}(-s, \check{\chi}),
$$

for $\check{\chi}(x)=\chi(\bar{x})^{-1}$, is defined by the integral

$$
M(s, \chi) \Phi(g)=\int_{\operatorname{Herm}_{n}(E)} \Phi(w n(x) g, s) d x .
$$


Here the measure $d x$ is normalized to be self dual with respect to the pairing $[x, y] \mapsto \psi(\operatorname{tr}(x y))$ on $\operatorname{Herm}_{n}(E)$. In particular, the additive character $\psi$ should really be included in the notation. For example, if we replace $\psi$ by $\psi_{a}$ for $a \in F^{\times}$, then $d x$, and hence $M(s, \chi)$, changes by a factor of $|a|_{F}^{n^{2}}$. Also, for $T \in \operatorname{Herm}_{n}(E)$, the generalized Whittaker integral is given by

$$
W_{T}(s) \Phi(g)=\int_{\mathrm{Herm}_{n}(E)} \Phi(w n(x) g, s) \psi(\operatorname{tr}(T x)) d x,
$$

with the same choice of measure. Karel [20] proved that, if $\operatorname{det} T \neq 0$, then $W_{T}(s)$ has an entire analytic continuation and a functional equation:

$$
W_{T}(-s) \circ M(s, \chi)=\kappa_{T}(s, \chi, \psi) \cdot W_{T}(s) .
$$

Note that $W_{T}(s)$ depends on the choice of $\psi$. The following result is proved in [25], and is analogous to a result of Sweet [44] for the symplectic group.

Proposition 6.3. Let $\chi^{o}=\left.\chi\right|_{F^{\times}}$. Then

$$
\begin{aligned}
\kappa_{T}(s, \chi, \psi)=\chi(\operatorname{det} T)^{-1} \mid & \left.\operatorname{det} T\right|_{F} ^{-2 s} \gamma_{F}(\Delta, \psi)^{\frac{n(n-1)}{2}} \\
& \times \epsilon_{E / F}(\operatorname{det} T)^{n-1} \prod_{r=0}^{n-1} \rho_{F}\left(2 s+r-n+1, \chi^{o} \cdot \epsilon_{E / F}^{r}, \psi\right) .
\end{aligned}
$$

Here, for a character $\xi$ of $F^{\times}, \rho_{F}(s, \xi, \psi)$ is Tate's gamma factor, [45] and (8.19) below, satisfying

$$
\rho_{F}(s, \xi, \psi)=\epsilon(s, \xi, \psi)^{-1} \frac{L(s, \xi)}{L\left(1-s, \xi^{-1}\right)} .
$$

(We have called it $\rho_{F}$ to avoid confusion with the Weil invariant $\gamma_{F}$.) Recall that it depends on the additive character $\psi$. Moreover, for $a \in F^{\times}$,

$$
\kappa_{T}\left(s, \chi, \psi_{a}\right)=|a|_{F}^{n^{2}} \kappa_{a T}(s, \chi, \psi)=\chi(a)^{-n}|a|_{F}^{-2 n s+n^{2}} \kappa_{T}(s, \chi, \psi) .
$$

We set

$$
\kappa(s, \chi, \psi)=\gamma_{F}(\Delta, \psi)^{\frac{n(n-1)}{2}} \prod_{r=0}^{n-1} \rho_{F}\left(2 s+r-n+1, \chi^{o} \cdot \epsilon_{E / F}^{r}, \psi\right),
$$

and define the normalized intertwining operator

$$
M^{*}(s, \chi)=M^{*}(s, \chi, \psi)=\frac{1}{\kappa(s, \chi, \psi)} \cdot M(s, \chi) .
$$

Note that

$$
M^{*}\left(s, \chi, \psi_{a}\right)=\chi(a)^{n}|a|_{F}^{2 n s} M^{*}(s, \chi, \psi) .
$$

We will usually suppress the dependence on $\psi$ from the notation. The functional equation (6.3) then becomes:

$$
W_{T}(-s) \circ M^{*}(s, \chi)=\chi(\operatorname{det} T)^{-1}|\operatorname{det} T|^{-s} \cdot \epsilon_{E / F}(\operatorname{det} T)^{n-1} \cdot W_{T}(s),
$$


and so, we obtain

$$
M^{*}(-s, \check{\chi}) \circ M^{*}(s, \chi)=1 .
$$

Note that all that has been done so far depends only on the group $U(n, n)$ and does not involve the embedding $i: G \times G \longrightarrow U(n, n)$.

Next recall that the local zeta integral is defined as follows. Let $\pi$ be an irreducible admissible representation of $G=G(W)$ and, for $\xi \in \pi$ and $\xi^{\vee} \in \pi^{\vee}$ let $\phi(g)=\left\langle\pi(g) \xi, \xi^{\vee}\right\rangle$ be the corresponding matrix coefficient. For a section $\Phi(s) \in I_{n}(s, \chi)$, define

$$
Z\left(s, \xi, \xi^{\vee}, \Phi\right)=\int_{G} \phi(g) \Phi(i(g, 1), s) d g .
$$

This integral converges for large $\operatorname{Re}(s)$ and defines a non-zero element

$$
Z(s) \in \operatorname{Hom}_{G \times G}\left(I_{n}(s, \chi), \pi^{\vee} \otimes(\chi \cdot \pi)\right) .
$$

To define the $\mathrm{L}$ and $\epsilon$-factors, Piatetski-Shapiro and Rallis introduce the family of good sections [33]. For a suitable choice of basis, the group $G\left(W+W_{-}\right)$is isomorphic to the subgroup of $G L(2 n, E)$ which preserves the skew-Hermitian form with matrix $\left(\begin{array}{cc}0 & 1_{n} \\ -1_{n} & 0\end{array}\right)$. Let $K$ be the maximal compact subgroup of $G\left(W+W_{-}\right)$ obtained by intersecting this group with $G L\left(2 n, \mathcal{O}_{E}\right)$. A section $\Phi(s)$ is standard if its restriction to $K$ is independent of $s$. For example, if $\chi$ is unramified, let $\Phi^{0}(s)$ be the standard section whose restriction to $K$ is equal to 1 . Recall that if $\chi$ and $\psi$ are unramified, then [28]

$$
M(s, \chi) \Phi^{0}(s)=\frac{a_{n}(s, \chi)}{b_{n}(s, \chi)} \cdot \Phi^{0}(-s),
$$

with

$$
a_{n}(s, \chi)=\prod_{r=0}^{n-1} L\left(2 s+r-n+1, \chi^{o} \epsilon_{E / F}^{r}\right),
$$

and

$$
b_{n}(s, \chi)=\prod_{r=0}^{n-1} L\left(2 s+n-r, \chi^{o} \epsilon_{E / F}^{r}\right),
$$

where $\chi^{o}$ is the restriction of $\chi$ to $F^{\times}$. Also, if $\chi$ and $\psi$ are unramified and ord $\operatorname{det} T=0$, then $[43]$

$$
W_{T}(s) \Phi^{0}(e)=\frac{1}{b_{n}(s, \chi)},
$$

where $W_{T}(s)$ is defined by (6.5).

Definition 6.4. The family of good sections consists of

(a) $\mathbb{C}\left[q^{s}, q^{-s}\right] \cdot$ standard sections,

(b) $M^{*}(-s, \check{\chi})\left(\mathbb{C}\left[q^{s}, q^{-s}\right] \cdot\right.$ standard sections $)$, 
and, when $\chi$ is unramified,

(c) the sections of the form

$$
b_{n}(s, \chi) \cdot \Phi^{0}(s) * \mu(s),
$$

where $\mu(s) \in \mathcal{H}\left(G\left(W+W_{-}\right), K\right)\left[q^{s}, q^{-s}\right]$, with $\mathcal{H}\left(G\left(W+W_{-}\right), K\right)$ the Hecke algebra with respect to $K$, which acts on the space of $K$-invariant sections by convolution on the right.

Lemma 6.5. (i) ([33])

$$
M^{*}(s, \chi)(\text { Good sections })=\text { Good sections. }
$$

(ii) Moreover,

$$
\kappa(s, \chi, \psi)=\gamma_{F}(\Delta, \psi)^{\frac{n(n-1)}{2}} \frac{a_{n}(s, \chi)}{b_{n}(-s, \check{\chi})} \cdot \prod_{r=0}^{n-1} \epsilon\left(2 s+r-n+1, \chi^{o} \epsilon_{E / F}^{r}, \psi\right)^{-1} .
$$

Proof. Since $M^{*}(s, \chi)((\mathrm{a}))=(\mathrm{b})$ and $M^{*}(s, \chi)((\mathrm{b}))=(\mathrm{a})$ by the functional equation (6.7), it suffices to consider

$$
\begin{aligned}
M^{*}(s, \chi)\left(b_{n}(s, \chi) \cdot \Phi^{0}(s) * \mu(s)\right) & =b_{n}(s, \chi) \frac{1}{\kappa(s, \chi, \psi)} \cdot \frac{a_{n}(s, \chi)}{b_{n}(s, \chi)} \Phi^{0}(-s) * \mu(s) \\
& =\frac{a_{n}(s, \chi)}{\kappa(s, \chi, \psi)} \Phi^{0}(-s) * \check{\mu}(-s) .
\end{aligned}
$$

But now,

$$
\begin{aligned}
& \frac{a_{n}(s, \chi)}{\kappa(s, \chi, \psi)} \\
& =\gamma_{F}(\Delta, \psi)^{-\frac{n(n-1)}{2}} \cdot \prod_{r=0}^{n-1} L\left(2 s+r-n+1, \chi^{o} \epsilon_{E / F}^{r}\right) \\
& \quad \times \frac{L\left(-2 s-r+n,\left(\chi^{o}\right)^{-1} \epsilon_{E / F}^{r}\right)}{L\left(2 s+r-n+1, \chi^{o} \epsilon_{E / F}^{r}\right)} \cdot \epsilon\left(2 s+r-n+1, \chi^{o} \epsilon_{E / F}^{r}, \psi\right) \\
& =b_{n}(-s, \check{\chi}) \cdot \gamma_{F}(\Delta, \psi)^{-\frac{n(n-1)}{2}} \cdot \prod_{r=0}^{n-1} \epsilon\left(2 s+r-n+1, \chi^{o} \epsilon_{E / F}^{r}, \psi\right) .
\end{aligned}
$$

The product of epsilon factors, etc., can be absorbed into the action of $\mathcal{H}\left[q^{s}, q^{-s}\right]$, so we find that $M^{*}(s, \chi)((\mathrm{c}))=(\mathrm{c})$, as desired.

Now, following [32], [33], (but notice that our normalization of the intertwining operator is different!) we have:

Proposition 6.6. (i) For any good section $\Phi(s), Z\left(s, \xi, \xi^{\vee}, \Phi\right) \in \mathbb{C}\left(q^{-s}\right)$, i.e., the zeta integral is a rational function of $q^{-s}$.

(ii) For any fixed $\xi$ and $\xi^{\vee}$, there exists a good section $\Phi(s)$ such that

$$
Z\left(s, \xi, \xi^{\vee}, \Phi(s)\right)=1 .
$$


(iii) The set

$$
\mathcal{I}=\left\{Z\left(s, \xi, \xi^{\vee}, \Phi\right) \mid \xi \in \pi, \xi^{\vee} \in \pi^{\vee}, \Phi(s)=\operatorname{good}\right\}
$$

is an ideal for $\mathbb{C}\left[q^{s}, q^{-s}\right]$, with a generator $P\left(q^{-s}\right)^{-1}$, for a unique polynomial $P(x) \in \mathbb{C}[x]$ with $P(0)=1$. Moreover, there exist $\xi, \xi^{\vee}$ and a good section $\Phi(s)$ such that

$$
Z\left(s, \xi, \xi^{\vee}, \Phi\right)=\frac{1}{P\left(q^{-s}\right)} .
$$

(iv) There is a functional equation

$$
Z\left(-s, \xi, \xi^{\vee}, M^{*}(s, \chi) \Phi\right)=\Gamma^{*}(s, \pi, \chi, \psi) \cdot Z\left(s, \xi, \xi^{\vee}, \Phi\right) \text {. }
$$

Recall that $M^{*}(s, \chi)=M^{*}(s, \chi, \psi)$ depends on $\psi$.

Now define

$$
L\left(s+\frac{1}{2}, \pi, \chi\right)=\frac{1}{P\left(q^{-s}\right)} .
$$

By (iv) and the functional equation (6.7) of $M^{*}(s, \chi)$,

$$
\Gamma^{*}(-s, \pi, \check{\chi}, \psi) \cdot \Gamma^{*}(s, \pi, \chi, \psi)=1 .
$$

Moreover, we have

$$
\frac{Z\left(-s, \xi, \xi^{\vee}, M^{*}(s, \chi) \Phi\right)}{L\left(\frac{1}{2}-s, \pi, \check{\chi}\right)}=\epsilon\left(s+\frac{1}{2}, \pi, \chi, \psi\right) \cdot \frac{Z\left(s, \xi, \xi^{\vee}, \Phi\right)}{L\left(s+\frac{1}{2}, \pi, \chi\right)},
$$

where

$$
\epsilon(s, \pi, \chi, \psi)=\frac{L(s, \pi, \chi)}{L(1-s, \pi, \check{\chi})} \cdot \Gamma^{*}\left(s-\frac{1}{2}, \pi, \chi, \psi\right) .
$$

Note that the functional equation

$$
\epsilon(1-s, \pi, \check{\chi}, \psi) \cdot \epsilon(s, \pi, \chi, \psi)=1
$$

follows from (6.21). By Lemma 6.5, the ratios on both sides of (6.22) are entire. Taking a section as in (iii) of Proposition 6.6 , we see that $\epsilon(s, \pi, \chi, \psi)$ is a rational function of $q^{-s}$ which is entire and never zero. Thus it is a monomial in $q^{-s}$. Finally, the functional equation (6.24) implies that

$$
\epsilon(s, \pi, \chi, \psi)=A \cdot q^{B\left(s-\frac{1}{2}\right)},
$$

for constants $A$ and $B$. Note that, when $\chi=\check{\chi}$, the combination of (6.24) and (6.25) yields $A= \pm 1$, i.e., statement (i) of Theorem 6.1 .

Before continuing, we observe that our definition of $\epsilon(s, \pi, \chi, \psi)$ has one desirable property. Specifically, for $a \in F^{\times}$, we have

$$
\epsilon\left(s, \pi, \chi, \psi_{a}\right)=\chi(a)^{n}|a|_{F}^{2 n\left(s-\frac{1}{2}\right)} \epsilon(s, \pi, \chi, \psi) .
$$

This follows from the analogous relation for $M^{*}(s, \chi, \psi)$. It is compatible with the comparison conjecture (0.19) and relation (1.3.9) in Jacquet [19] for the standard $\epsilon$-factor for $G L_{n}(E)$, provided we recall that $|a|_{E}=|a|_{F}^{2}$ and that the central character of $B C(\pi) \otimes \chi$ restricts to $\chi^{n}$ on $F^{\times}$.

Proof of (ii) of Theorem 6.1. Our goal is to relate the dichotomy of Corollary 4.4 to the root number $\epsilon\left(\frac{1}{2}, \pi, \chi, \psi\right)$ defined by doubling. We first note the following facts. 
Lemma 6.7. Fix the character $\chi$.

(i) [25] The operator

$$
M^{* *}(s, \chi)=\frac{1}{a_{n}(s, \chi)} M(s, \chi)
$$

is entire; $M^{* *}\left(s_{0}, \chi\right)$ is not identically zero for any value of $s_{0}$. Also, by (ii) of Lemma 6.4,

$$
M^{*}(s, \chi)=(\text { entire }) \cdot b_{n}(-s, \check{\chi}) \cdot M^{* *}(s, \chi) .
$$

In particular, for a standard section $\Phi(s)$, the poles of $M^{*}(-s, \check{\chi}) \Phi$ lie among the poles of $b_{n}(s, \chi)$.

(ii) The poles of the good sections lie among the poles of $b_{n}(s, \chi)$.

(iii) $b_{n}(s, \chi)$ is holomorphic at $s=0$ for any (unitary) $\chi$, while, if $\left.\chi\right|_{F \times}=\epsilon_{E / F}^{n}$, then $a_{n}(s, \chi)$ is holomorphic at $s=0$. In particular, when $\left.\chi\right|_{F^{\times}}=\epsilon_{E / F}^{n}$ then $M(s, \chi)$ is holomorphic at $s=0$.

(iv) For any good section $\Phi(s)$, there is a neighborhood of $s=0$ on which $\Phi(s)$ is a linear combination of standard sections with coefficients holomorphic functions of $s$.

Proof of (i) of Theorem 4.3. For any irreducible admissible representation $\pi$ of $G(W)$, and for any fixed $s_{0} \in \mathbb{C}$, the zeta integral defines an intertwining map:

$$
\begin{aligned}
Z^{*}\left(s_{0}\right): I_{n}\left(s_{0}, \chi\right) & \longrightarrow \pi^{\vee} \otimes(\chi \cdot \pi), \\
\Phi & \mapsto\left(\left.\xi \otimes \xi^{\vee} \mapsto \frac{Z\left(s, \xi, \xi^{\vee}, \Phi\right)}{L\left(s+\frac{1}{2}, \pi, \chi\right)}\right|_{s=s_{0}}\right),
\end{aligned}
$$

where an element $\Phi \in I_{n}\left(s_{0}, \chi\right)$ is first extended to a standard section $\Phi(s) \in$ $I_{n}(s, \chi)$. If the relation analogous to that of (iv) of Lemma 6.7 holds for good sections and standard sections at $s=s_{0}$, then the order of pole at $s=s_{0}$ of the family $Z\left(s, \xi, \xi^{\vee}, \Phi\right)$ for good sections $\Phi(s)$ coincides with that for the family $Z\left(s, \xi, \xi^{\vee}, \Phi\right)$ for standard sections $\Phi(s)$. Thus, by the definition of the L-factor, it follows that $Z^{*}\left(s_{0}\right)$ is a non-zero element of $\operatorname{Hom}_{G \times G}\left(I_{n}\left(s_{0}, \chi\right), \pi^{\vee} \otimes(\chi \cdot \pi)\right)$. On the other hand, suppose that the family $Z\left(s, \xi, \xi^{\vee}, \Phi\right)$ for good sections has a higher order pole at $s=s_{0}$ than the family $Z\left(s, \xi, \xi^{\vee}, \Phi\right)$ for standard sections $\Phi(s)$, so that $Z^{*}\left(s_{0}\right)$ will be identically zero. In this case, we can replace $Z^{*}\left(s_{0}\right)$ by the leading term of Laurent expansion of the family

$$
\frac{Z\left(s, \xi, \xi^{\vee}, \Phi\right)}{L\left(s+\frac{1}{2}, \pi, \chi\right)}
$$

for $\Phi(s)$ a standard section. This leading term again defines a non-zero intertwining map, and (i) of Theorem 4.3 is proved.

We now specialize (6.27) to the case $s_{0}=0$. If we assume that $\left.\chi\right|_{F^{\times}}=\epsilon_{E / F}^{n}$, then, by (iv) of Lemma 6.7, the order of pole at $s=0$ of the family $Z\left(s, \xi, \xi^{\vee}, \Phi\right)$ for good sections $\Phi(s)$ coincides with that for the family $Z\left(s, \xi, \xi^{\vee}, \Phi\right)$ for standard sections $\Phi(s)$. Thus, by the definition of the L-factor, it follows that $Z^{*}(0)$ is a nonzero element of $\operatorname{Hom}_{G \times G}\left(I_{n}(0, \chi), \pi^{\vee} \otimes(\chi \cdot \pi)\right)$. We want to consider the restriction of $Z^{*}(0)$ to the subspaces $R_{n}\left(V_{n}^{ \pm}, \chi\right)$. For this we note that the functional equation (6.22) implies that

$$
Z^{*}(0) \circ M^{*}(0, \chi)=\epsilon\left(\frac{1}{2}, \pi, \chi, \psi\right) \cdot Z^{*}(0)
$$


On the other hand, by (6.11),

$$
M^{*}(0, \chi)^{2}=1 .
$$

Since the $R_{n}\left(V_{n}^{ \pm}, \chi\right)$ 's are irreducible and non-isomorphic submodules of $I_{n}(0, \chi)$, $M^{*}(0, \chi)$ must act by a scalar on each of them.

Proposition 6.8.

$$
\left.M^{*}(0, \chi)\right|_{R_{n}(V, \chi)}=\epsilon_{E / F}(-2)^{n} \cdot \epsilon_{E / F}(\operatorname{det} V) .
$$

Proof. By (6.10), we have

$$
W_{T}(0) \circ M^{*}(0, \chi)=\epsilon_{E / F}(\operatorname{det} T) \cdot W_{T}(0) .
$$

On the other hand, $W_{T}(0)$ is non-zero on $R_{n}(V, \chi)$ for $V=V_{-2 T}$, the Hermitian space of dimension $n$ defined by the Hermitian matrix $T$, while it is identically zero on the other Hermitian space of dimension $n$, [25]. Thus we find that

$$
\left.M^{*}(0, \chi)\right|_{R_{n}\left(V_{-2 T}, \chi\right)}=\epsilon_{E / F}(\operatorname{det} T),
$$

as claimed.

Comparing this result with the functional equation (6.29), we obtain:

\section{Proposition 6.9.}

$$
\left.Z^{*}(0)\right|_{R_{n}(V, \chi)} \neq 0 \Longleftrightarrow \epsilon\left(\frac{1}{2}, \pi, \chi, \psi\right)=\epsilon_{E / F}(-2)^{n} \cdot \epsilon_{E / F}(\operatorname{det} V) .
$$

Combining this result with Proposition 3.1, we obtain the second statement of Theorem 6.1.

Proof of Theorem 6.2. Since the matrix coefficients of a supercuspidal representation $\pi$ are compactly supported, the only poles of $Z\left(s, \xi, \xi^{\vee}, \Phi\right)$ must arise from the poles of the good section $\Phi(s)$. By (ii) of Lemma 6.7, these must lie among the poles of $b_{n}(s, \chi)$.

Lemma 6.10. Assume that the pair $(s, \chi)$ is normalized, and write $\chi^{o}=\left.\chi\right|_{F^{\times}}$.

(i) If $\chi \neq \check{\chi}$, then $b_{n}(s, \chi)$ is entire.

(ii) If $\left.\chi\right|_{F^{\times}}=\check{\chi}$, then the poles of $b_{n}(s, \chi)$ are simple and occur at the points

$$
X_{n}(\chi)=\left\{\frac{m-n}{2} \mid 0 \leq m \leq n-1, \chi^{o}=\epsilon_{E / F}^{m}\right\} .
$$

Now suppose that $\chi=\check{\chi}$ is fixed and that $s_{0}=\frac{m-n}{2} \in X_{n}(\chi)$. Then, in a neighborhood of $s_{0}$, any good section can be written in the form

$$
\Phi(s)=b_{n}(s, \chi) \sum_{j} \alpha_{j}(s) \Psi_{j}(s),
$$

where the $\Psi_{j}(s)$ are standard sections and the $\alpha_{j}(s)$ are holomorphic functions. Moreover, if $\alpha_{j}\left(s_{0}\right) \neq 0$, then

$$
\Psi_{j}\left(s_{0}\right) \in R_{n}\left(V_{m}^{+}, \chi\right) \oplus R_{n}\left(V_{m}^{-}, \chi\right) \subset I_{n}\left(s_{0}, \chi\right) .
$$


Here we note that, by (iii) of Proposition 4.1, the submodule $R_{n}\left(V_{m}^{+}, \chi\right) \oplus R_{n}\left(V_{m}^{-}, \chi\right)$ is the image of the intertwining operator $M^{* *}\left(-s_{0}, \chi\right)$. Moreover, if $\chi$ is unramified, this submodule contains the spherical vector $\Phi^{0}\left(s_{0}\right)$. Thus, the family of zeta integrals $Z\left(s, \xi, \xi^{\vee}, \Phi\right)$ has a pole at $s=s_{0}$ if and only if there is a standard section $\Psi(s)$ with $\Psi\left(s_{0}\right) \in R_{n}\left(V_{m}^{+}, \chi\right) \oplus R_{n}\left(V_{m}^{-}, \chi\right)$ such that

$$
Z\left(s_{0}, \xi, \xi^{\vee}, \Psi\right) \neq 0 .
$$

But the restriction of $Z\left(s_{0}, \cdot, \cdot, \cdot\right)$ to

$$
\pi \otimes \pi^{\vee} \otimes\left(R_{n}\left(V_{m}^{+}, \chi\right) \oplus R_{n}\left(V_{m}^{-}, \chi\right)\right)
$$

defines an element of

$$
\operatorname{Hom}_{G \times G}\left(R_{n}\left(V_{m}^{+}, \chi\right) \oplus R_{n}\left(V_{m}^{-}, \chi\right), \pi^{\vee} \otimes(\chi \cdot \pi)\right) .
$$

This space is at most one dimensional, by Corollary 4.2 and Theorem 4.3 , and it is easy to see that the element defined by $Z\left(s_{0}, \cdot, \cdot, \cdot\right)$ is, in fact, a basis. Applying Proposition 3.1, we obtain the assertion of Theorem 6.2.

We conclude this section with some remarks on the way in which the epsilon factor defined by doubling depends on the Hermitian space $W$. First recall that the discussion of $(6.3)-(6.11)$ takes place on the group $U(n, n)$, and hence is independent of the embedding $i: G(W) \times G(W) \longrightarrow U(n, n)$.

For $\alpha \in F^{\times}$, let $W_{\alpha}$ denote the space $W$ with skew-Hermitian form $\alpha\langle$,$\rangle . If$ $\epsilon_{E / F}(\alpha)=-1$, i.e., if $\alpha \notin N_{E / F} E^{\times}$, and if $n$ is odd, then the skew-Hermitian spaces $W_{\alpha}$ and $W$ are not isomorphic, although their isometry groups $G=G(W)=G\left(W_{\alpha}\right)$ are canonically identified. We obtain the diagram:

$$
\begin{aligned}
& i: \quad G(W) \times G(W) \quad \longrightarrow G(W+\bar{W}) \quad \longrightarrow \quad U(n, n) \\
& \|\quad\| \quad \downarrow A d\left(c_{\alpha}\right) \\
& i_{\alpha}: G\left(W_{\alpha}\right) \times G\left(W_{\alpha}\right) \longrightarrow G\left(W_{\alpha}+\bar{W}_{\alpha}\right) \longrightarrow U(n, n),
\end{aligned}
$$

where, for a suitable choice of bases,

$$
c_{\alpha}=\left(\begin{array}{cc}
1_{n} & \\
& \alpha \cdot 1_{n}
\end{array}\right) .
$$

Note that $A d\left(c_{\alpha}\right)$ preserves the Siegel parabolic $P$ and carries $I_{n}(s, \chi)$ to itself, i.e., if $\Phi(s) \in I_{n}(s, \chi)$, then the function

$$
\Phi^{\alpha}(g, s)=\Phi\left(c_{\alpha} g c_{\alpha}^{-1}, s\right)
$$

also lies in $I_{n}(s, \chi)$. For a matrix coefficient $\phi$ of $\pi$ and for a section $\Phi(s) \in I_{n}(s, \chi)$, let

$$
Z_{\alpha}(s, \phi, \Phi)=\int_{G} \phi(g) \Phi\left(i_{\alpha}(g, 1), s\right) d g=Z\left(s, \phi, \Phi^{\alpha}\right) .
$$

From the family of zeta integrals $Z_{\alpha}(s, \phi, \Phi)$ obtained by letting $\Phi(s)$ run over the space of good sections of Definition 6.4, we obtain factors $L_{\alpha}(s, \pi, \chi)$ and $\epsilon_{\alpha}(s, \pi, \chi, \psi)$. 
Proposition 6.11. (i) For any $\chi$,

$$
\Gamma_{\alpha}^{*}(s, \pi, \chi, \psi)=\chi(\alpha)^{n}|\alpha|_{F}^{2 n s} \Gamma^{*}(s, \pi, \chi, \psi) .
$$

In particular, if $L(s, \pi, \chi)$ and $L_{\alpha}(s, \pi, \chi)$ have poles at $s=\frac{1}{2}$ whose orders have the same parity, e.g., if $\pi$ is supercuspidal, then

$$
\epsilon_{\alpha}(1 / 2, \pi, \chi, \psi)=\chi(\alpha)^{n} \epsilon(1 / 2, \pi, \chi, \psi)
$$

(ii) Suppose that $\chi$ is ramified. Then

$$
L_{\alpha}(s, \pi, \chi)=L(s, \pi, \chi)
$$

and

$$
\epsilon_{\alpha}(s, \pi, \chi, \psi)=\chi(\alpha)^{n}|\alpha|_{F}^{2 n\left(s-\frac{1}{2}\right)} \epsilon(s, \pi, \chi, \psi) .
$$

Thus, if $n$ is odd and if $\left.\chi\right|_{F^{\times}}=\epsilon_{E / F}$, the root numbers $\epsilon(1 / 2, \pi, \chi, \psi)$ of $\pi$, defined by the doubling method, vary with $\alpha$ as $\pi$ is viewed as a representation of $G\left(W_{\alpha}\right)$ !

In fact, we expect that the condition that $\chi$ be ramified can be dropped. Note that this result is compatible with the comparison conjecture (0.18) and (0.19).

Proof. We begin with (ii). If $\chi$ is ramified, then the good sections are just those defined by (a) and (b) of Definition 6.4.

Lemma 6.12. (i) If

$$
\Phi(s) \in \mathbb{C}\left[q^{s}, q^{-s}\right] \cdot(\text { standard sections }),
$$

then

$$
\Phi^{\alpha}(s) \in \mathbb{C}\left[q^{s}, q^{-s}\right] \cdot(\text { standard sections }) .
$$

(ii) If

$$
\Phi(s) \in M^{*}(s, \chi)\left(\mathbb{C}\left[q^{s}, q^{-s}\right] \cdot(\text { standard sections })\right)
$$

then

$$
\Phi^{\alpha}(s) \in M^{*}(s, \chi)\left(\mathbb{C}\left[q^{s}, q^{-s}\right] \cdot(\text { standard sections })\right) .
$$

(iii)

$$
M(s, \chi) \Phi^{\alpha}=\chi(\alpha)^{-1}|\alpha|_{F}^{-2 n s}(M(s, \chi) \Phi)^{\alpha}
$$


Proof. Let

$$
K^{\prime}=K \cap c_{\alpha}^{-1} K c_{\alpha}
$$

and write

$$
K=\coprod_{i}(P \cap K) k_{i} K^{\prime}
$$

with $k_{i} \in K$. Also write

$$
c_{\alpha} k_{i} c_{\alpha}^{-1}=n_{i} m\left(a_{i}\right) \tilde{k}_{i} .
$$

For a standard section $\Phi(s) \in I_{n}(s, \chi)$, and for each index $i$, define a standard section $\Phi_{i}^{\alpha}(s)$ with

$$
\operatorname{supp}\left(\Phi_{i}^{\alpha}(s)\right)=P k_{i} K^{\prime}
$$

and

$$
\Phi_{i}^{\alpha}\left(k_{i} k^{\prime}, s\right)=\Phi\left(\tilde{k}_{i} c_{\alpha} k^{\prime} c_{\alpha}^{-1}, s\right)
$$

Note that the second expression here is independent of $s$, since $\Phi(s)$ is standard. Then

$$
\Phi^{\alpha}(s)=\sum_{i} \chi\left(\operatorname{det} a_{i}\right)\left|\operatorname{det} a_{i}\right|^{s+\rho_{n}} \cdot \Phi_{i}^{\alpha}(s),
$$

and so, in particular, $\Phi^{\alpha}(s)$ lies in $\mathbb{C}\left[q^{s}, q^{-s}\right] \cdot$ ( standard sections ), as claimed in (i).

Next we prove (iii), from which (ii) follows immediately. We have:

$$
\begin{aligned}
M(s, \chi) \Phi^{\alpha}(g) & =\int_{N} \Phi^{\alpha}(w n(b) g, s) d b \\
& =\int_{N} \Phi\left(c_{\alpha} w n(b) g c_{\alpha}^{-1}, s\right) d b \\
& =\int_{N} \Phi\left(m\left(\alpha^{-1}\right) w n\left(\alpha^{-1} b\right) c_{\alpha} g c_{\alpha}^{-1}, s\right) d b \\
& =\chi(\alpha)^{-n}|\alpha|_{F}^{-2 n\left(s+\frac{n}{2}\right)}|\alpha|_{F}^{n^{2}} \int_{N} \Phi\left(w n(b) c_{\alpha} g c_{\alpha}^{-1}, s\right) d b \\
& =\chi(\alpha)^{-n}|\alpha|_{F}^{-2 n s}(M(s, \chi) \Phi)^{\alpha}(g),
\end{aligned}
$$

as was to be shown.

Thus, if $\chi$ is ramified, we have shown that

$$
(\operatorname{good} \text { sections })^{\alpha}=(\operatorname{good} \text { sections }) \text {, }
$$

and so, by (6.41), we find the claimed relation between the L-factors. 
Next we make no assumptions about $\chi$ and consider the functional equation:

$$
Z_{\alpha}\left(-s, \phi, M^{*}(s, \chi) \Phi\right)=\Gamma_{\alpha}^{*}(s, \pi, \chi, \psi) Z_{\alpha}(s, \phi, \Phi)=\Gamma_{\alpha}^{*}(s, \pi, \chi, \psi) Z\left(s, \phi, \Phi^{\alpha}\right) .
$$

The left side equals:

$$
\begin{aligned}
Z\left(-s, \phi,\left(M^{*}(s, \chi) \Phi\right)^{\alpha}\right) & =\chi(\alpha)^{n}|\alpha|_{F}^{2 n s} Z\left(-s, \phi, M^{*}(s, \chi) \Phi^{\alpha}\right) \\
& =\chi(\alpha)^{n}|\alpha|_{F}^{2 n s} \Gamma^{*}(s, \pi, \chi, \psi) Z\left(s, \phi, \Phi^{\alpha}\right) .
\end{aligned}
$$

Here we assume that $\Phi(s)$ is a standard section, so that we may apply (6.48). Comparing these expressions, we find that

$$
\Gamma_{\alpha}^{*}(s, \pi, \chi, \psi)=\chi(\alpha)^{n}|\alpha|_{F}^{2 n s} \Gamma^{*}(s, \pi, \chi, \psi),
$$

as claimed in (i). If $\chi$ is ramified, we have already shown that the L-factors do not change with the scaling by $\alpha$, and thus, via (6.23), we obtain the behavior of the $\epsilon$ factor under such scaling claimed in (ii).

If $\chi$ is arbitrary, and if we assume that the two L-factors have orders at $s=\frac{1}{2}$ of the same parity, then the result on the root number claimed in (i) again follows from (6.52) and (6.23).

\section{§7. FunCtORIALity}

In this section we review the, mostly conjectural, functoriality properties of the local theta correspondence. In the unitary group case, conjectures about the behavior of A-packets under the correspondence were made by J. Adams [1], and also by Gelbart and Rogawski [6]. After reviewing these conjectures, we hazard a sightly more refined guess about the L-packets involved. We then illustrate this guess in certain very special cases and finally explain what it would say about the L-parameters of the supercuspidals occuring in the 'curious example' of section 5.

Throughout this section, we let

$$
W_{F}^{\prime}=S L_{2}(\mathbb{C}) \times W_{F} \quad \text { and } \quad W_{F}^{\prime \prime}=W_{F}^{\prime} \times S L_{2}(\mathbb{C}),
$$

where $W_{F}$ is the Weil group of $F$. Also, for a character $\mu$ of $E^{\times}$, we write $\mu^{o}$ for the restriction $\left.\mu\right|_{F^{\times}}$.

First, recall that if $H=G(V)$ is a unitary group, with $\operatorname{dim}_{E} V=m$, then

$$
{ }^{L} H=G L_{m}(\mathbb{C}) \rtimes W_{F},
$$

where the subgroup $W_{E}$ of $W_{F}$ acts trivially, and an element $w_{\sigma} \in W_{F}-W_{E}$ acts on ${ }^{\vee} H=G L_{m}(\mathbb{C})$ by

$$
g \mapsto \Phi_{m}^{t} g^{-1} \Phi_{m}^{-1}
$$

where

$$
\Phi_{m}=\left(\begin{array}{cccc} 
& & & 1 \\
& \ddots & & \\
(-1)^{m-1} & & &
\end{array}\right) .
$$


We fix an element $w_{\sigma} \in W_{F}-W_{E}$ once and for all. For a Langlands parameter

$$
\varphi: W_{F}^{\prime} \longrightarrow{ }^{L} H
$$

we will write $\Pi_{\varphi}$ for the (conjecturally) corresponding L-packet. These are, of course, known for $U(1), U(2)$ and $U(3)$ [41]. In the case $m=1$, for a character $\eta$ of $U(1) \simeq E^{1}$, the norm 1 subgroup of $E^{\times}$, we let $\tilde{\eta}$ be the character of $E^{\times}$obtained by pullback:

$$
\tilde{\eta}(x)=\eta(x / \bar{x}) .
$$

Then, for $\varphi: W_{F} \longrightarrow \mathbb{C}^{\times} \rtimes W_{F}$ defined by

$$
\varphi: w \mapsto \tilde{\eta}(w) \times w \quad \text { and } \quad w_{\sigma} \mapsto 1 \times w_{\sigma},
$$

$\Pi_{\varphi}=\{\eta\}$. The cases of $U(2)$ (due essentially to [26]) and $U(3)$ (due to [41], Chapter 13) are discussed in Chapters 11 and 12-13 of [41].

Finally, if

$$
\psi: W_{F}^{\prime \prime} \longrightarrow{ }^{L} H
$$

is an Arthur parameter [2], we let $\Pi_{\psi}$ be the (conjecturally) corresponding Arthur packet. Again, these are known for $U(1)$ (trivial), $U(2)$ and $U(3)$, and are discussed in detail in [27]. Recall that for a given Arthur parameter $\psi$, there is a corresponding Langlands parameter

$$
\varphi_{\psi}: W_{F}^{\prime} \longrightarrow{ }^{L} H
$$

defined by composing $\psi$ with the map $W_{F}^{\prime} \longrightarrow W_{F}^{\prime \prime}$ given by

$$
g_{1} \times w \mapsto g_{1} \times w \times\left(\begin{array}{ll}
|w|^{\frac{1}{2}} & \\
& |w|^{-\frac{1}{2}}
\end{array}\right) .
$$

Here recall that, according to our convention, the second $S L_{2}(\mathbb{C})$ is added last.

We recall from [41, Chapter 4, 4.6] that for a unitary group $H=G(V)$, where $\operatorname{dim}_{E} V=m$, the elliptic endoscopic groups are the quasi-split unitary groups $I=U(a) \times U(b)$, where $a+b=m$. The embedding ${ }^{L} I \longrightarrow{ }^{L} H$ depends on the choice of characters $\mu_{1}$ and $\mu_{2}$ of $E^{\times}$satisfying:

$$
\mu_{1}^{o}=\epsilon_{E / F}^{b} \quad \text { and } \quad \mu_{2}^{o}=\epsilon_{E / F}^{a} .
$$

Then

$$
\begin{aligned}
\left(g_{1}, g_{2}\right) \times 1 & \mapsto\left(\begin{array}{ll}
g_{1} & \\
& g_{2}
\end{array}\right) \times 1, \\
1 \times w & \mapsto\left(\begin{array}{ll}
\mu_{1}(w) & \\
& \mu_{2}(w)
\end{array}\right) \times w,
\end{aligned}
$$

where $w \in W_{E}$, and

$$
1 \times w_{\sigma} \mapsto \Phi_{a, b} \cdot \Phi_{m}^{-1} \times w_{\sigma}
$$


Note that the conditions on $\mu_{1}^{o}$ and $\mu_{2}^{o}$ are 'crossed'. Here

$$
\Phi_{a, b}=\left(\begin{array}{cc}
\Phi_{a} & \\
& \Phi_{b}
\end{array}\right) .
$$

Now suppose that $G=G(W)$ and $H=G(V)$ are given, with $W$ skew-Hermitian, $\operatorname{dim}_{E} W=n$, and $V$ Hermitian with $\operatorname{dim}_{E} V=m$. Assume that $m \geq n$, and let $I=U(a) \times U(b)$ be the elliptic endoscopic group of $H$ with $a=n$ and $b=m-n$. We define an L-homomorphism ${ }^{L} G \longrightarrow{ }^{L} I$ by

$$
\begin{aligned}
g \times 1 & \mapsto\left(g, 1_{b}\right) \times 1, \\
1 \times w & \mapsto 1 \times w
\end{aligned}
$$

and $1 \times w_{\sigma} \mapsto 1 \times w_{\sigma}$. Composing this with the map ${ }^{L} I \longrightarrow{ }^{L} H$ defined above, we obtain a map $\alpha_{m, n}$,

$$
\begin{aligned}
\alpha_{m, n}:{ }^{L} G & \longrightarrow{ }^{L} H, \\
g \times 1 & \mapsto\left(\begin{array}{ll}
g & \\
& 1_{b}
\end{array}\right) \times 1, \\
1 \times w & \mapsto\left(\begin{array}{ll}
\mu_{1}(w) & \\
& \mu_{2}(w)
\end{array}\right) \times w
\end{aligned}
$$

and

$$
1 \times w_{\sigma} \mapsto \Phi_{a, b} \cdot \Phi_{m}^{-1} \times w_{\sigma} .
$$

This map plays a key role in Adams' conjecture.

One additional ingredient is needed. For any $b \in \mathbb{Z}_{\geq 1}$, let $\rho_{b}$ be the symmetric tensor representation of $S L_{2}(\mathbb{C})$ on $S y m^{b-1}\left(\mathbb{C}^{2}\right)$. Note that $\operatorname{dim}_{\mathbb{C}} \rho_{b}=b$. We take the monomial basis

$$
\frac{(b-1) !}{(b-i-1) !} x^{b-i-1} y^{i}
$$

$0 \leq i \leq b-1$, for $S y m^{b-1}\left(\mathbb{C}^{2}\right)$, and hence have $\rho_{b}: S L_{2}(\mathbb{C}) \longrightarrow G L_{b}(\mathbb{C})$.

Lemma 7.1. For all $g \in S L_{2}(\mathbb{C})$,

$$
\Phi_{b}^{-1}\left({ }^{t} \rho_{b}(g)^{-1}\right) \Phi_{b}=\rho_{b}(g) .
$$

In particular, the representation $\rho_{b}$ is symplectic for $b$ even and orthogonal for $b$ odd.

Now suppose that $\chi_{1}$ and $\chi_{2}$ are characters of $E^{\times}$with $\chi_{1}^{o}=\epsilon_{E / F}^{m}$ and $\chi_{2}^{o}=\epsilon_{E / F}^{n}$, so that we obtain a splitting homomorphism

$$
\tilde{\iota}_{V, \chi_{1}} \times \tilde{\iota}_{W, \chi_{2}}: G(W) \times G(V) \longrightarrow M p(\mathbb{W}),
$$

as described in section 1 and in the appendix. For simplicity, we will often write $\chi$ for the pair of characters $\left(\chi_{1}, \chi_{2}\right)$. 
Conjecture 7.2 (Adams' conjecture). Suppose that $\psi: W_{F}^{\prime \prime} \longrightarrow{ }^{L} G$ is an Arthur parameter for $G$ and define an Arthur parameter

$$
\theta_{\chi}(\psi): W_{F}^{\prime \prime} \longrightarrow{ }^{L} H
$$

for $H$ by

$$
\left.\theta_{\chi}(\psi)\right|_{W_{F}^{\prime}}=\left.\alpha_{\chi} \circ \psi\right|_{W_{F}^{\prime}},
$$

and

$$
\left.\theta_{\chi}(\psi)\right|_{1 \times 1 \times S L_{2}(\mathbb{C})}=\left(\left.\alpha_{\chi} \circ \psi\right|_{1 \times 1 \times S L_{2}(\mathbb{C})}\right) \oplus \rho_{b} .
$$

Here $\alpha_{\chi}$ is the L-homomorphism defined above with

$$
\mu_{1}=\chi_{1}^{-1} \chi_{2},
$$

and

$$
\mu_{2}=\chi_{2} .
$$

This is well defined, i.e., the image of $w_{\sigma}$ commutes with the image of the second $S L_{2}(\mathbb{C})$ by Lemma 7.1. Suppose that $\pi$ is an irreducible admissible representation of $G(W)$ with $\pi \in \Pi_{\psi}$. Then

$$
\theta_{\chi}(\pi, V) \in \Pi_{\theta_{\chi}(\psi)} .
$$

Note that, if $m-n \geq 2$, the parameter $\theta_{\chi}(\psi)$ will be non-trivial on Arthur's $S L_{2}(\mathbb{C})$, even if the original parameter is trivial on this factor. This corresponds to the fact that the theta lift of a tempered representation may be non-tempered.

In fact, Adams [1] states his conjecture in terms of E-groups, rather than Lgroups, and works directly with the representations of the full inverse images of $G(W)$ and $G(V)$ in $M p(\mathbb{W})$, or rather its twofold analogue. Thus he avoids the choice of the pair of characters $\chi$ needed to define the splitting homomorphism and the choice of the pair of characters $\left(\mu_{1}, \mu_{2}\right)$ needed to define the L-homomorphism. He also gives a more intrinsic description of the 'tail' $\rho_{b}$, as the representation of $S L_{2}(\mathbb{C})$ associated to the principal unipotent conjugacy class in the centralizer of the image under $\alpha$ of $\hat{G}$. Our description is more convenient for the calculations we have at hand, and it is an amusing exercise to check that the two versions of the conjecture agree.

A similar surmise about the relation of the theta correspondence to endoscopy was made by Gelbart and Rogawski [6, p. 448], based on their results in the cases $(U(1), U(1)),(U(1), U(3))$ and $(U(2), U(3))$.

First we observe that the relationship we have given between the pairs $\chi=$ $\left(\chi_{1}, \chi_{2}\right)$ and $\mu=\left(\mu_{1}, \mu_{2}\right)$ is more or less determined. Specifically, suppose that we change $\chi_{i}$ to $\chi_{i} \nu_{i}$ for characters $\nu_{1}$ and $\nu_{2}$ of $E^{\times}$which are trivial on $F^{\times}$. Let $\nu_{i}^{\prime}, i=1,2$, denote the corresponding characters of $E^{1}$. Then the splitting homomorphism associated to $\chi^{\prime}$ differs from that associated to $\chi$ by the character $\nu^{\prime}=\nu_{1}^{\prime} \otimes \nu_{2}^{\prime}$ of $G(W) \times G(V)$, and hence the Weil representations satisfy

$$
S_{\chi^{\prime}}=\nu^{\prime} \otimes S_{\chi}
$$


Thus

$$
\begin{aligned}
\sigma_{2}=\theta_{\chi}\left(\sigma_{1}, V\right) & \Longleftrightarrow \operatorname{Hom}_{G(W) \times G(V)}\left(S_{\chi}, \sigma_{1} \otimes \sigma_{2}\right) \neq 0 \\
& \Longleftrightarrow \operatorname{Hom}_{G(W) \times G(V)}\left(S_{\chi^{\prime}},\left(\nu_{1}^{\prime} \sigma_{1}\right) \otimes\left(\nu_{2}^{\prime} \sigma_{2}\right)\right) \neq 0 \\
& \Longleftrightarrow \nu_{2}^{\prime} \sigma_{2}=\theta_{\chi^{\prime}}\left(\nu_{1}^{\prime} \sigma_{1}, V\right) .
\end{aligned}
$$

This determines how the groups of characters of $G(W) \times G(V)$ must act on our parameters.

Remark. It only remains to check that we have chosen the correct base point. This can be done by considering the correspondence on irreducible principal series and using the compatibility with parabolic induction. In particular, using the standard methods of [37], [21], and the analysis of splittings given in [22] and the appendix to this article, it is easy to verify that the conjecture of Adams is true when $\pi$ is an irreducible principal series representation and the theta correspondence is defined using an unramified additive character. In particular, the characters $\mu_{1}$ and $\mu_{2}$ are correct in this case. We omit the details.

Next we present some speculations about the actual L-parameters involved. We phrase these as speculations rather than as conjectures, since we have not tested them in sufficiently general situations. Throughout this discussion we will assume the Howe duality conjecture, and write $\theta_{\chi}(\pi, V)$ for the unique irreducible quotient of $\Theta_{\chi}(\pi, V)$ (or for 0 if the irreducible representation $\pi$ of $G(W)$ does not occur in the $\chi$-theta correspondence with $V$ ). We will also grant the existence of L-packets in the extended sense described by Vogan [48]. Thus, for a given Langlands parameter $\varphi: W_{F}^{\prime} \longrightarrow{ }^{L} G$, the packet $\Pi_{\varphi}$ will $^{1}$ be a finite set of irreducible admissible representations, some of which may be representations of $G^{+}=G\left(W^{+}\right)$and some of $G^{-}=G\left(W^{-}\right)$, where $W^{ \pm}$are the two possible skew-Hermitian spaces of dimension $n$. Moreover, the representations in the set $\Pi_{\varphi}$ will be parameterized by the irreducible characters, taken up to a certain equivalence, of the finite group $A_{\varphi}$, where $A_{\varphi}=C_{\varphi} / C_{\varphi}^{0}$, for $C_{\varphi}$ the centralizer in ${ }^{\vee} G$ of the image of $\varphi$. The precise description is given in Vogan [48], p. 344, Definition 4.14 and Conjecture 4.15. In our present case, ${ }^{\vee} G=G L_{n}(\mathbb{C})$, so that $Z\left({ }^{\vee} G\right) \simeq \mathbb{C}^{\times}$,

$$
Z\left({ }^{\vee} G\right)^{\Gamma} \simeq\langle \pm 1\rangle
$$

and

$$
H^{1}(\Gamma, G) \stackrel{\sim}{\longrightarrow}\left(Z\left({ }^{\vee} G\right)^{\Gamma}\right)^{\wedge} \simeq(\langle \pm 1\rangle)^{\wedge} .
$$

There is a natural map

$$
Z\left({ }^{\vee} G\right)^{\Gamma} \longrightarrow A_{\varphi}
$$

and, after suitable choice of base point (Vogan [48] pp. 345-346), the representation $\pi$ attached to the irreducible representation $\xi$ of $A_{\varphi}$ lives on the unitary group $G\left(W^{\epsilon}\right)$, where $\epsilon=\xi(-1)$ is the value of $\xi$ on the image in $A_{\varphi}$ of the non-trivial element in $Z\left({ }^{\vee} G\right)^{\Gamma}$.

We will use the same convention for the groups $G\left(V^{ \pm}\right)$.

We will make essential use of the involution $\psi \leftrightarrow \psi^{\prime}$ on the set of Arthur parameters, mentioned in [27]. The parameter $\pi^{\prime}$ is obtained by composing $\psi$ with the $\operatorname{map} s w: W_{F}^{\prime \prime} \longrightarrow W_{F}^{\prime \prime}$,

$$
s w: g_{1} \times w \times g_{2} \mapsto g_{2} \times w \times g_{1} .
$$

\footnotetext{
${ }^{1}$ We are now granting the conjectures...
} 
Fix the pair of skew-Hermitian spaces $W_{n}^{ \pm}$of dimension $n$, and consider the two Witt towers $\left\{V_{m}^{+}\right\}$and $\left\{V_{m}^{-}\right\}$with $\operatorname{dim}_{E} V_{m}^{ \pm}=m$. Here we use an obvious variant of the notation introduced at the beginning of section 5. Let $G_{n}^{ \pm}=G\left(W_{n}^{ \pm}\right)$ and let $H_{m}^{ \pm}=G\left(V_{m}^{ \pm}\right)$. Fix the pair of characters $\chi$ used to define the splitting homomorphism and the theta correspondence for all of the dual pairs $\left(G_{n}^{+}, H_{m}^{ \pm}\right)$ and $\left(G_{n}^{-}, H_{m}^{ \pm}\right)$.

Speculation 7.3. If $m=n$, then the theta correspondence is functorial, i.e., if $\pi$ lies in an (extended) L-packet $\Pi_{\varphi}\left(G_{n}\right)$ and if $\chi_{1}=\chi_{2}$, then $\theta_{\chi}\left(\pi, V_{n}^{ \pm}\right)$lies in the (extended) L-packet $\Pi_{\varphi}\left(H_{n}\right)$.

Speculation 7.4. If $m=n$, then for any irreducible admissible $\pi$, precisely one of the spaces $\theta_{\chi}\left(\pi, V_{n}^{+}\right)$and $\theta_{\chi}\left(\pi, V_{n}^{-}\right)$is non-zero. (If $\pi$ is supercuspidal, this is Corollary 4.4.) The sign $\epsilon(\pi)= \pm 1$ for which $\theta_{\chi}\left(\pi, V_{n}^{\epsilon(\pi)}\right) \neq 0$ may depend on $\chi$, and upon the inner form $G_{n}^{ \pm}$of which $\pi$ is a representation, but is otherwise independent of the choice of $\pi$ within the extended packet. Thus, if $\chi_{1}=\chi_{2}=\chi$, then the map

$$
\pi \mapsto \theta_{\chi}\left(\pi, V_{n}^{\epsilon(\pi)}\right)
$$

permutes the elements of the (extended) L-packet. In particular, this permutation either carries

$$
\Pi_{\varphi}\left(G_{n}^{+}\right) \leftrightarrow \Pi_{\varphi}\left(G_{n}^{+}\right) \quad \text { and } \quad \Pi_{\varphi}\left(G_{n}^{-}\right) \leftrightarrow \Pi_{\varphi}\left(G_{n}^{-}\right)
$$

or

$$
\Pi_{\varphi}\left(G_{n}^{+}\right) \leftrightarrow \Pi_{\varphi}\left(G_{n}^{-}\right) \quad \text { and } \quad \Pi_{\varphi}\left(G_{n}^{-}\right) \leftrightarrow \Pi_{\varphi}\left(G_{n}^{+}\right)
$$

Note that the second case can occur only when

$$
\left|\Pi_{\varphi}\left(G_{n}^{+}\right)\right|=\left|\Pi_{\varphi}\left(G_{n}^{-}\right)\right| .
$$

Suppose that $\varphi$ is a fixed Langlands parameter for $G$. For $\pi$ in the L-packet $\Pi_{\varphi}$, suppose that $\theta_{\chi}\left(\pi, V_{n}^{+}\right) \neq 0$, and hence that $\theta_{\chi}\left(\pi, V_{n}^{-}\right)=0$. In this case, we say that $\pi$ occurs late in the (-)-tower. How late it occurs should be determined by how early it occurs in the $(+)$-tower:

Speculation 7.5. Let $m_{0}^{+}$(resp. $m_{0}^{-}$) be the smallest value of $m$, of the same parity as $n$, such that $\theta_{\chi}\left(\pi, V_{m}^{+}\right) \neq 0\left(\right.$ resp. $\left.\theta_{\chi}\left(\pi, V_{m}^{-}\right) \neq 0\right)$. Then

$$
m_{0}^{+}+m_{0}^{-}=2 n+2 \text {. }
$$

Moreover, the pair $\left\{m_{0}^{+}, m_{0}^{-}\right\}$depends only on the L-packet $\Pi_{\varphi}$.

Note that the idea that $\left\{m_{0}^{+}, m_{0}^{-}\right\}$depends on $\varphi$ alone is motivated by (ii) of Theorem 6.2. 
Remark. In the case of $m$ and $n$ odd, so that the groups $G^{+}=G\left(W_{n}^{+}\right)$and $G^{-}=$ $G\left(W_{n}^{-}\right)$can be canonically identified, a given representation $\pi$ of this group should occur twice in the (extended) packet, once as $\pi^{+}$, a representation of $G_{n}^{+}$, and once as $\pi^{-}$, a representation of $G_{n}^{-}$. For fixed $\chi$, these two should have non-zero theta lifts to opposite towers on the $V$ side, and should account for the same "multiplicity 2' phenomenon for $G\left(V_{n}^{+}\right)=G\left(V_{n}^{-}\right)$. For a given supercuspidal $\pi$, this disjunction follows from Theorem 6.1 and Proposition 6.11.

For example, one might expect that for most representations,

$$
\left\{m_{0}^{+}, m_{0}^{-}\right\}=\{n, n+2\},
$$

i.e., most representations do not show up in the theta correspondence until they are forced to, by Corollary 4.5, and this delays their appearance only one step in the 'late' tower.

The following simple example is instructive. Consider groups

$$
\begin{array}{cccc}
G_{3}^{+} & G_{3}^{-} & H_{3}^{+} & H_{3}^{-} \\
G_{1}^{+} & G_{1}^{-} & H_{1}^{+} & H_{1}^{-} .
\end{array}
$$

For any character $\eta$ of $E^{1}$, the extended L-packet consists of the pair $\left\{\eta^{+}, \eta^{-}\right\}$, where $\eta^{ \pm}$is the character $\eta$ of $G_{1}^{ \pm}$. We assume that the characters $\chi_{1}=\chi_{2}=\chi$, with $\chi^{o}=\epsilon_{E / F}$, are fixed, and we let

$$
\epsilon=\epsilon\left(\frac{1}{2}, \chi \tilde{\eta}^{-1}, \psi_{E}\right) \eta(-1) \chi(-\delta)
$$

be the sign given by Corollary 8.5. Then, if $\epsilon=1$, we have the following picture:

$$
\begin{array}{cccc}
\theta_{\chi}\left(\eta^{+}, W_{3}^{+}\right) & \theta_{\chi}\left(\eta^{-}, W_{3}^{-}\right) & \theta_{\chi}\left(\eta^{+}, V_{3}^{+}\right) & \theta_{\chi}\left(\eta^{-}, V_{3}^{-}\right) \\
\theta_{\chi}\left(\eta^{-}, W_{3}^{+}\right) & \theta_{\chi}\left(\eta^{+}, W_{3}^{+}\right) & \theta_{\chi}\left(\eta^{-}, V_{3}^{+}\right) & \theta_{\chi}\left(\eta^{+}, V_{3}^{-}\right) \\
\eta^{+} & \eta^{-} & \eta^{+}=\theta_{\chi}\left(\eta^{+}, V_{1}^{+}\right) & \eta^{-}=\theta_{\chi}\left(\eta^{-}, V_{1}^{-}\right) .
\end{array}
$$

The representations in the top two rows may be identified as:

$$
\begin{array}{llll}
\pi^{n,+} & \pi^{n,-} & \pi^{n,+} & \pi^{n,-} \\
\pi^{s,+} & \pi^{s,-} & \pi^{s,+} & \pi^{s,-}
\end{array}
$$

where $\pi^{n, \pm}$ is the non-tempered constituent of the principal series associated to the character

$$
\left(\begin{array}{ccc}
a & & \\
& b & \\
& & \bar{a}^{-1}
\end{array}\right) \mapsto \chi(a)|a|_{E}^{\frac{1}{2}} \eta(b),
$$


and viewed as a representation of the group $G_{3}^{ \pm}$or $H_{3}^{ \pm}$. In Rogawski [41, pp.198199], this representation is denoted by $\pi^{n}(\xi)$, for a suitable $\xi$. Similarly, $\pi^{s, \pm}$ is the supercuspidal representation, denoted by $\pi^{s}(\xi)$ in Rogawski [41], Proposition 13.1.3 (d), p.199, where $\xi$ is determined by the condition that $\left\{\pi^{n}(\xi), \pi^{s}(\xi)\right\}$ is the Apacket discussed on p. 199, and, again, viewed as a representation of the group $G_{3}^{ \pm}$ or $H_{3}^{ \pm}$.

Finally, let $\pi^{2, \pm}\left(\pi^{2}(\xi)\right.$ in [41]) be the square integrable constituent of the principal series containing $\pi^{n, \pm}\left(\pi^{n}(\xi)\right.$ in [41]). Then the collection of representations

$$
\theta_{\chi}\left(\pi^{s,-}, W_{3}^{+}\right) \quad \theta_{\chi}\left(\pi^{s,+}, W_{3}^{-}\right) \quad \theta_{\chi}\left(\pi^{s,-}, V_{3}^{+}\right) \quad \theta_{\chi}\left(\pi^{s,+}, V_{3}^{-}\right),
$$

coincides with

$$
\pi^{2,+} \quad \pi^{2,-} \quad \pi^{2,+} \quad \pi^{2,-} .
$$

Observe that $\left\{\pi^{n,+}, \pi^{n,-}\right\}$ is the extended L-packet associated to the non-tempered parameter

$$
\begin{aligned}
\varphi^{n}: W_{F}^{\prime} & \longrightarrow G L_{3}(\mathbb{C}) \rtimes W_{F}, \\
w & \mapsto\left(\begin{array}{rr}
\tilde{\eta}(w) & \\
\chi(w)|w|^{\frac{1}{2}} & \\
& \chi(w)|w|^{-\frac{1}{2}}
\end{array}\right) \times w, \\
w_{\sigma} & \mapsto \Phi_{1,2} \Phi_{3}^{-1} \times w_{\sigma}
\end{aligned}
$$

and trivial on $S L_{2}(\mathbb{C})$. Note that this parameter is obtained by applying Arthur's recipe, (7.8) and (7.9), to the A-parameter given by Adams' conjecture.

Similarly,

$$
\left\{\pi^{s,+}, \pi^{2,+}, \pi^{s,-}, \pi^{2,-}\right\}
$$

is the extended discrete series L-packet associated to the parameter

$$
\begin{aligned}
\varphi^{2}: W_{F}^{\prime} & \longrightarrow G L_{3}(\mathbb{C}) \rtimes W_{F}, \\
w & \mapsto\left(\begin{array}{ccc}
\tilde{\eta}(w) & \\
& \chi(w) & \\
& \chi(w)
\end{array}\right) \times w, \\
w_{\sigma} & \mapsto \Phi_{1,2} \Phi_{3}^{-1} \times w_{\sigma},
\end{aligned}
$$

and

$$
g \mapsto\left(\begin{array}{cc}
1 & \\
& g
\end{array}\right) \times 1
$$


where $g \in S L_{2}(\mathbb{C})$. Note that this parameter is obtained by applying the involution (7.20) on Arthur parameters to the parameter of Adams' conjecture. The resulting Arthur parameter is trivial on the second $S L_{2}(\mathbb{C})$, and hence is just an L-parameter. It turns out to be precisely the L-parameter we want.

The situation when the sign $\epsilon$ of (7.23) is -1 is similar, with the obvious switch in signs.

Next we consider the case in which $m$ and $n$ have opposite parity. Here we recall that Corollary 4.5 asserts that, for any irreducible admissible representation $\pi$ of $G_{n}^{ \pm}$, at least one of the spaces $\theta_{\chi}\left(\pi, V_{n+1}^{+}\right)$and $\theta_{\chi}\left(\pi, V_{n+1}^{-}\right)$is non-zero.

Speculation 7.6. Let $m_{0}^{+}$(resp. $m_{0}^{-}$) be the smallest value of $m$, of parity opposite to that of $n$, such that $\theta_{\chi}\left(\pi, V_{m}^{+}\right) \neq 0\left(\right.$ resp. $\left.\theta_{\chi}\left(\pi, V_{m}^{-}\right) \neq 0\right)$. Then

$$
m_{0}^{+}+m_{0}^{-}=2 n+2 .
$$

Moreover, the pair $\left\{m_{0}^{+}, m_{0}^{-}\right\}$depends only on the L-packet $\Pi_{\varphi}$.

Thus, for most L-packets, we would have $m_{0}^{+}=m_{0}^{-}=n+1$. At the other extreme, for $n$ odd and for the trivial representation $\mathbb{1}$, we have $m_{0}^{+}=0$, since $\mathbb{1}$ comes from the $U(0)$ which lies, formally, at the bottom of the tower of 'split' unitary groups. Thus, Speculation 7.6 predicts that $m_{0}^{-}=2 n+2$, so that $\theta_{\chi}\left(\mathbb{1}, V_{2 r}^{-}\right)=0$ for $r=1,2, \ldots, n$. Here we are assuming that $\chi_{1}=1$. In fact, the vanishing of $\theta_{\chi}\left(\mathbb{1}, V_{2 r}^{-}\right)$in this range has now been proved, [24].

These speculations are not difficult to verify for the dual pairs $(U(1), U(m)), m$ even, and $(U(2), U(m)), m$ odd.

Next, we describe a guess about the parameters involved in the 'curious example' of section 5. We assume that $\chi_{1}=\chi_{2}=\chi$ is fixed with $\chi^{o}=\epsilon_{E / F}^{n}$. For this, we may suppose that $\pi=\pi_{0}$ is a supercuspidal representation of $G=G\left(W^{+}\right)$, $\operatorname{dim}_{E} W=n$ for which (7.21) holds. Thus $\theta_{\chi}\left(\pi, V_{n}^{+}\right)$(say) is supercuspidal, and $\theta_{\chi}\left(\pi, V_{n}^{-}\right)=0$. According to Speculation 7.3, the L-parameter of $\theta_{\chi}\left(\pi, V_{n}^{+}\right)$is the same as that of $\pi_{0}$. On the other hand, according to Speculation 7.5 and (7.21), $\pi_{1}=\theta_{\chi}\left(\pi_{0}, V_{n+2}^{-}\right) \neq 0$ and is a supercuspidal representation of $G\left(V_{n+2}^{-}\right)$. Applying Speculation 7.5 again, we obtain $\pi_{2}=\theta_{\chi}\left(\pi_{1}, V_{n+6}^{-}\right)$, etc. Let $\varphi_{0}: W_{F}^{\prime} \longrightarrow{ }^{L} G_{n}$ be the L-parameter for $\pi_{0}$. Then

Speculation 7.7. Suppose that $\pi$ is a supercuspidal of $G_{n}$ for which (7.21) holds. The following list gives the L-parameters of the sequence of supercuspidals constructed from $\pi$ :

$$
\begin{array}{ccc}
n & \pi_{0} & \varphi_{0} \\
n+2 & \pi_{1} & \varphi_{0} \oplus \chi \cdot \rho_{2} \\
n+6 & \pi_{2} & \varphi_{0} \oplus \chi\left(\rho_{2} \oplus \rho_{4}\right) \\
n+12 & \pi_{3} & \varphi_{0} \oplus \chi\left(\rho_{2} \oplus \rho_{4} \oplus \rho_{6}\right) \\
\ldots & \ldots & \ldots \\
n+r(r+1) & \pi_{r} & \varphi_{0} \oplus \chi\left(\rho_{2} \oplus \rho_{4} \oplus \ldots \rho_{2 r}\right) \\
\ldots & \ldots & \ldots
\end{array}
$$


Here the parameter $\varphi_{r}$ for $\pi_{r}$ is given, more precisely, by:

$$
\begin{aligned}
\varphi_{r}: W_{F}^{\prime} & \longrightarrow{ }^{L} G_{n+r(r+1)}, \\
w & \mapsto\left(\begin{array}{cc}
\varphi_{00}(w) & \\
& \chi(w) \cdot 1_{r(r+1)}
\end{array}\right) \times w, \\
w_{\sigma} & \mapsto\left(\begin{array}{ll}
\varphi_{00}\left(w_{\sigma}\right) & \\
& 1_{r(r+1)}
\end{array}\right) \cdot \Phi_{n, 2,4, \ldots, 2 r} \cdot \Phi_{n+r(r+1)}^{-1} \times w_{\sigma},
\end{aligned}
$$

and

$$
g \mapsto\left(\begin{array}{ccccc}
\varphi_{00}(g) & & & & \\
& \rho_{2}(g) & & & \\
& & \rho_{4}(g) & & \\
& & & \ddots & \\
& & & & \rho_{2 r}(g)
\end{array}\right) \times 1 .
$$

Here $\varphi_{0}(w)=\varphi_{00}(w) \times w$.

Speculation 7.7 is compatible with Theorem 6.2. Observe that for the supercuspidal $\pi_{r}$ of $G_{n+r(r+1)}$, the analogue of (7.21) is

$$
\left\{m_{0}^{+}\left(\pi_{r}\right), m_{0}^{-}\left(\pi_{r}\right)\right\}=\{n+r(r-1), n+r(r+3)+2\} .
$$

By Theorem 6.2, the fact that the earliest theta lift of $\pi_{r}$ is to $G_{n+r(r-1)}$ means that

$$
L\left(s, \pi_{r}^{\vee}, \chi\right)=\left(1-q_{E}^{-\left(s+\frac{1}{2}\right)}\right)^{-1}\left(1-q_{E}^{-\left(s+\frac{3}{2}\right)}\right)^{-1} \ldots\left(1-q_{E}^{-\left(s+\frac{2 r-1}{2}\right)}\right)^{-1} .
$$

Note that the condition (7.21) on $\pi=\pi_{0}$ implies that

$$
L\left(s, \pi_{0}^{\vee}, \chi\right)=1,
$$

since the earliest theta lift is to $G_{n}^{ \pm}$. On the other hand, the L-parameter for $\pi_{r}^{\vee}$ is

$$
\varphi_{0}^{\vee} \oplus \chi^{-1}\left(\rho_{2} \oplus \rho_{4} \oplus \cdots \oplus \rho_{2 r}\right) .
$$

But, by [46], for example,

$$
L\left(s, \chi^{-1} \rho_{b}, \chi\right)=\left(1-q_{E}^{-\left(s+\frac{b-1}{2}\right)}\right)^{-1} .
$$

Thus the L-factor attached to the restriction of our L-parameter to $W_{E}^{\prime}$ and to the standard representation of the L-group $G L_{n+r(r+1)}(\mathbb{C}) \times W_{E}$ agrees with the doubling L-factor of $\pi_{r}$, as determined by Theorem 6.2 .

The phenomena involving $\mathrm{L}$ and A-packets in the example discussed in (7.22)(7.33) above should have an analogue in the general case.

Remark. The $L$ - parameter $\phi_{r}$ of the representation $\pi_{r}$ introduced in Speculation 7.7 contains each of the representations $\rho_{i}, i=2,4, \ldots, 2 r$, with multiplicity one. Let $\phi$ be an $L$-parameter in which one of the $\rho_{i}$ occurs with multiplicity greater than one. Then, as one easily verifies, the image of $\phi$ is contained in a proper Levi subgroup of the corresponding L-group. The full Langlands functoriality conjecture implies that such $\phi$ is not associated to a discrete series L-packet and, a fortiori, does not define a supercuspidal representation. In other words, the sequence of dimensions $n, n+2, n+6, \ldots, n+r(r+1)$ proposed in Speculation 7.7 is the "densest" compatible with the Langlands conjectures. 
The following is the analogue of Speculation 7.7 in the case of opposite parity. Note that, in this case, chains of supercuspidals once again exist, due to the dichotomy of Corollary 4.4. The 'bottom' of such a chain occurs when $\pi$ is a supercuspidal representation of $G_{n}$ such that

$$
m_{0}^{+}=m_{0}^{-}=n+1 \text {. }
$$

The steps in the chain are determined by Speculation 7.6, so that we obtain a chain of supercuspidals $\pi_{r}, r=0,1, \ldots$ on $G_{m}$ 's with $m$ given by

$$
n, n+1, n+4, n+9, \ldots, n+r^{2}, \ldots
$$

Recall that, in this case, we must fix a pair of characters $\chi_{1}$ and $\chi_{2}$ of $E^{\times}$such that $\chi_{1}^{o}=\epsilon_{E / F}^{n+1}$ and $\chi_{2}^{o}=\epsilon_{E / F}^{n}$. We use $\chi=\left(\chi_{1}, \chi_{2}\right)$ to define the splittings and theta lifts for the dual pairs $\left(G_{n}, G_{n+1}\right),\left(G_{n+4}, G_{n+9}\right)$, etc. and we use $\chi=\left(\chi_{2}, \chi_{1}\right)$ to define the splittings and theta lifts for the dual pairs $\left(G_{n+1}, G_{n+4}\right),\left(G_{n+9}, G_{n+16}\right)$, etc.

Speculation 7.8. Suppose that $\pi$ is a supercuspidal of $G_{n}$ for which (7.39) holds. The following list gives the L-parameters of the sequence of supercuspidals constructed from $\pi$ :

$$
\begin{array}{ccc}
n & \pi_{0} & \varphi_{0} \\
n+1 & \pi_{1} & \left(\chi_{1}^{-1} \chi_{2} \cdot \varphi_{0}\right) \oplus \chi_{2} \cdot \rho_{1} \\
n+4 & \pi_{2} & \varphi_{0} \oplus \chi_{1}\left(\rho_{1} \oplus \rho_{3}\right) \\
n+9 & \pi_{3} & \left(\chi_{1}^{-1} \chi_{2} \cdot \varphi_{0}\right) \oplus \chi_{2}\left(\rho_{1} \oplus \rho_{3} \oplus \rho_{5}\right) \\
\ldots & \ldots & \ldots \\
n+r^{2} & \pi_{r} & \left\{\begin{array}{c}
\chi_{2} \\
\chi_{1}
\end{array}\right\} \cdot\left(\left(\chi_{1}^{-1} \cdot \varphi_{0}\right) \oplus \rho_{1} \oplus \rho_{3} \oplus \cdots \oplus \rho_{2 r-1}\right) \\
\ldots & \ldots & \ldots
\end{array}
$$

Here the parameter $\varphi_{r}$ for $\pi_{r}$ is given, more precisely, by:

$$
\begin{aligned}
\varphi_{r}: W_{F}^{\prime} & \longrightarrow{ }^{L} G_{n+r^{2}}, \\
w & \mapsto \chi_{j}(w) \cdot\left(\begin{array}{ll}
\chi_{1}(w)^{-1} \varphi_{00}(w) & \\
& 1_{r^{2}}
\end{array}\right) \times w, \\
w_{\sigma} & \mapsto\left(\begin{array}{ll}
\varphi_{00}\left(w_{\sigma}\right) & \\
& 1_{r^{2}}
\end{array}\right) \cdot \Phi_{n, 1,3, \ldots, 2 r-1} \cdot \Phi_{n+r^{2}}^{-1} \times w_{\sigma},
\end{aligned}
$$

and

$$
g \mapsto\left(\begin{array}{ccccc}
\varphi_{00}(g) & & & & \\
& \rho_{1}(g) & & & \\
& & \rho_{3}(g) & & \\
& & & \ddots & \\
& & & & \rho_{2 r-1}(g)
\end{array}\right) \times 1 .
$$

Here $\varphi_{0}(w)=\varphi_{00}(w) \times w$, and $\chi_{j}=\chi_{1}$ for $r$ even and $\chi_{2}$ for $r$ odd.

The analogues of (7.34)-(7.36) are:

$$
\left\{m_{0}^{+}\left(\pi_{r}\right), m_{0}^{-}\left(\pi_{r}\right)\right\}=\left\{n+(r-1)^{2}, n+(r+1)^{2}\right\},
$$




$$
L\left(s, \pi_{r}^{\vee}, \chi_{j}\right)=\left(1-q_{E}^{-s}\right)^{-1}\left(1-q_{E}^{-(s+1)}\right)^{-1} \ldots\left(1-q_{E}^{-(s+r-1)}\right)^{-1},
$$

and

$$
L\left(s, \pi_{0}^{\vee}, \chi_{1}\right)=1,
$$

where, in (7.42), $\chi_{j}=\chi_{1}$ for $r$ even and $\chi_{2}$ for $r$ odd. Thus, we again have compatibility between Theorem 6.2 and our conjectural Langlands parameter.

\section{§8. Comparison of $\epsilon$-Factors, the Case $n=1$}

In this section we restrict to the case of $U(1)$ and we determine the relation between the $\epsilon$-factor defined by the doubling method and the usual Tate $\epsilon$-factor of the base change. In fact we give a complete treatment of the (local) doubling construction in this case.

To be more precise, let $W=E$ with the skew-Hermitian form $\langle x, y\rangle=\delta x \bar{y}$, and let $G=G(W)=E^{1}$ be the associated unitary group. We identify the split skewHermitian space $W+W_{-}$with the standard split skew-Hermitian space $E^{2}$ with skew-Hermitian inner product defined by $\left(\begin{array}{ll}-1 & 1\end{array}\right)$, and obtain the embedding

$$
i: G \times G \longrightarrow U(1,1)=H .
$$

Specializing the discussion of section 6 , we find that the normalizing factor for the intertwining operator reduces to

$$
\kappa(s, \chi, \psi)=\rho\left(2 s, \chi_{0}, \psi\right),
$$

and that

$$
a_{1}(s, \chi)=L\left(2 s, \chi_{0}\right)
$$

and

$$
b_{1}(s, \chi)=L\left(2 s+1, \chi_{0}\right) .
$$

Let $P \subset H$ be the Siegel parabolic. Then $P \backslash H$ consists of a single $i(G \times G)$ orbit (!), so that we may write

$$
H=P \cdot i(G \times 1) .
$$

Given any character $\eta$ of $G=E^{1}$, there is a unique section $\Phi_{\eta}(s)$ defined by

$$
\Phi_{\eta}(n m(a) i(g, 1), s)=\chi(a)|a|_{E}^{s+\frac{1}{2}} \eta(g) .
$$

The restriction of the degenerate principal series representation $I_{1}(s, \chi)$ to $i(G \times 1)$ then decomposes as:

$$
I(s)=\bigoplus_{\eta} \mathbb{C} \cdot \Phi_{\eta}(s)
$$

and the function $\Phi_{\eta}(s)$ transforms according to the character $\eta \otimes\left(\eta^{\vee} \cdot \chi\right)$ of $G \times G$. Moreover,

Lemma 8.1. $\Phi_{\eta}(s)$ is an eigenfunction for the intertwining operator, with

$$
M(s, \chi) \Phi_{\eta}(s)=\mu(s, \chi, \eta) \cdot \Phi_{\eta}(-s),
$$


where

$$
\mu(s, \chi, \eta)=\chi(-1) \int_{F} \chi^{-1} \tilde{\eta}\left(b+\frac{1}{2 \delta}\right)\left|b+\frac{1}{2 \delta}\right|_{E}^{-s-\frac{1}{2}} d b,
$$

and $\tilde{\eta}(x)=\eta\left(\frac{x}{\bar{x}}\right)$.

Proof. For large $\operatorname{Re}(s)$, we have:

$$
\mu(s, \chi, \eta)=M(s, \chi) \Phi_{\eta}(e)=\int_{F} \Phi_{\eta}(w n(b), s) d b .
$$

We calculate the decomposition

$$
\left(\begin{array}{ll} 
& 1 \\
-1 &
\end{array}\right)\left(\begin{array}{ll}
1 & b \\
& 1
\end{array}\right)=\left(\begin{array}{ll}
1 & x \\
& 1
\end{array}\right)\left(\begin{array}{ll}
\alpha & \\
& \bar{\alpha}^{-1}
\end{array}\right) \cdot i(g, 1) .
$$

Recall that

$$
i(g, 1)=\frac{1}{2}\left(\begin{array}{cc}
g+1 & (2 \delta)^{-1}(g-1) \\
(2 \delta)(g-1) & g+1
\end{array}\right),
$$

so that, comparing bottom rows, we have

$$
(-1,-b)=\bar{\alpha}^{-1}\left(\delta(g-1), \frac{1}{2}(g+1)\right) .
$$

This gives

$$
\alpha=-\frac{1}{b-1 / 2 \delta}
$$

and

$$
g=\frac{b+1 / 2 \delta}{b-1 / 2 \delta}
$$

and thus

$$
\begin{aligned}
\Phi_{\eta}(w n(b), s) & =\chi(-1) \cdot \chi^{-1}\left(b+\frac{1}{2 \delta}\right)\left|b+\frac{1}{2 \delta}\right|_{E}^{-s-\frac{1}{2}} \eta\left(\frac{b+1 / 2 \delta}{b-1 / 2 \delta}\right) \\
& =\chi(-1) \cdot \chi^{-1} \tilde{\eta}\left(b+\frac{1}{2 \delta}\right)\left|b+\frac{1}{2 \delta}\right|_{E}^{-s-\frac{1}{2}}
\end{aligned}
$$

with $\tilde{\eta}(x)=\eta\left(\frac{x}{\bar{x}}\right)$. Therefore

$$
\mu(s, \chi, \eta)=\chi(-1) \int_{F} \chi^{-1} \tilde{\eta}\left(b+\frac{1}{2 \delta}\right)\left|b+\frac{1}{2 \delta}\right|_{E}^{-s-\frac{1}{2}} d b,
$$

as claimed.

Proposition 8.2. Let $\mu$ be a character of $E^{\times}$and write $\mu_{0}=\left.\mu\right|_{F^{\times}}$. Then

$$
\int_{F} \mu(a+2 \delta)|a+2 \delta|_{E}^{-s-\frac{1}{2}} d a=\mu(4 \delta)|4 \delta|_{E}^{-s} \frac{\rho\left(2 s, \mu_{0}^{-1}, \psi\right)}{\rho\left(s+\frac{1}{2}, \mu^{-1}, \psi_{E}\right)},
$$

where $\rho(s, \lambda, \psi)$ is the Tate 'gamma' factor.

Recall that, for any character $\lambda$ of $F^{\times}, \rho(s, \lambda, \psi)$ is defined by the relation

$$
\rho(s, \lambda, \psi) Z_{T}\left(1-s, \lambda^{-1}, \hat{f}\right)=Z_{T}(s, \lambda, f),
$$

where

$$
Z_{T}(s, \lambda, f)=\int_{F} f(x) \lambda(x)|x|_{F}^{s-1} d x,
$$


for a self dual measure $d x$ with respect to $\psi$, and $\hat{f}$ the Fourier transform with respect to $\psi$ and this measure. The Tate factor for $E$ is defined analogously. Normalizing by the Tate L-factor, we have:

$$
\frac{Z_{T}\left(1-s, \lambda^{-1}, \hat{f}\right)}{L\left(1-s, \lambda^{-1}\right)}=\frac{1}{\rho(s, \lambda, \psi)} \frac{L(s, \lambda)}{L\left(1-s, \lambda^{-1}\right)} \cdot \frac{Z_{T}(s, \lambda, f)}{L(s, \lambda)},
$$

and so

$$
\frac{1}{\rho(s, \lambda, \psi)}=\epsilon(s, \lambda, \psi) \cdot \frac{L\left(1-s, \lambda^{-1}\right)}{L(s, \lambda)},
$$

where $\epsilon(s, \lambda, \psi)$ is the Tate epsilon factor.

Proof. Write

$$
B(s, \mu)=\int_{F} \mu(a+2 \delta)|a+2 \delta|_{E}^{-s-\frac{1}{2}} d a,
$$

and note that this integral is absolutely convergent for $\operatorname{Re}(s)>\frac{1}{2}$. Choose (nonzero) Schwartz functions $f \in S(F)$ and $\phi \in S(E)$ and let $\hat{f}$ (resp. $\hat{\phi}$ ) denote the Fourier transform with respect to $\psi$ (resp. $\psi_{E}$ ). We take Haar measures $d x$ on $F$ and $d y$ on $E$ to be self dual with respect to these fixed additive characters. We further assume that $\hat{f}(0)=0$ and $\hat{\phi}(0)=0$.

Consider the product

$$
Z_{T}\left(-2 s+1, \mu_{0}, \hat{f}\right) \cdot B(s, \mu) \cdot Z_{T}\left(s+\frac{1}{2}, \mu^{-1}, \phi\right),
$$

where $Z_{T}$ denotes the Tate zeta integral. If we assume that $s$ lies in the half plane $\operatorname{Re}(s)>\frac{1}{2}$, then we may write the product of the three factors as:

$$
\int_{F} \hat{f}(b) \mu_{0}(b)|b|_{F}^{-2 s} d b \cdot \int_{F} \mu(a+2 \delta)|a+2 \delta|_{E}^{-s-\frac{1}{2}} d a \cdot \int_{E} \phi(y) \mu^{-1}(y)|y|^{s-\frac{1}{2}} d y .
$$

Note that the condition $\hat{f}(0)=0$ insures that the first of these integrals is absolutely convergent for all $s$. Since

$$
\begin{aligned}
& \int_{F} \mu(a+2 \delta b)|a+2 \delta b|_{E}^{-s-\frac{1}{2}} d a \\
= & \int_{F} \mu(a b+2 \delta b)|a b+2 \delta b|_{E}^{-s-\frac{1}{2}} d(a b) \\
= & \mu(b)|b|_{F}^{-2 s-1}|b|_{F} \int_{F} \mu(a+2 \delta)|a+2 \delta|_{E}^{-s-\frac{1}{2}} d a \\
= & \mu_{0}(b)|b|_{F}^{-2 s} \int_{F} \mu(a+2 \delta)|a+2 \delta|_{E}^{-s-\frac{1}{2}} d a,
\end{aligned}
$$

we find that the product of the first two factors can be written as

$$
\int_{F} \int_{F} \hat{f}(b) \mu(a+2 \delta b)|a+2 \delta b|_{E}^{-s-\frac{1}{2}} d a d b .
$$

Setting $z=a+2 \delta b$, we can write this as

$$
\begin{aligned}
C \cdot \int_{E} \hat{f}\left(\frac{1}{4} \operatorname{tr}_{E / F}\right. & \left.\left(\delta^{-1} z\right)\right) \mu(z)|z|_{E}^{-s-\frac{1}{2}} d z \\
& =C \cdot \mu(2 \delta)|2 \delta|_{E}^{-s+\frac{1}{2}} \int_{E} \hat{f}\left(\frac{1}{2} \operatorname{tr} E / F(z)\right) \mu(z)|z|_{E}^{-s-\frac{1}{2}} d z,
\end{aligned}
$$


for a positive constant $C$, defined by $C \cdot d z=d a d b$, which we must still determine.

Dropping the factor $C \cdot \mu(2 \delta)|2 \delta|_{E}^{-s+\frac{1}{2}}$ for the moment, we observe that,

$$
\begin{aligned}
& \int_{E} \hat{f}\left(\frac{1}{2} \operatorname{tr}_{E / F}(y z)\right) \mu(z)|z|_{E}^{-s-\frac{1}{2}} d z \\
= & \int_{E} \hat{f}\left(\frac{1}{2} \operatorname{tr}_{E / F}(z)\right) \mu\left(y^{-1} z\right)\left|y^{-1} z\right|_{E}^{-s-\frac{1}{2}} d\left(y^{-1} z\right) \\
= & \mu^{-1}(y)|y|_{E}^{s-\frac{1}{2}} \int_{E} \hat{f}\left(\frac{1}{2} \operatorname{tr}_{E / F}(z)\right) \mu(z)|z|_{E}^{-s-\frac{1}{2}} d z,
\end{aligned}
$$

so that we may write the full product as:

$$
\begin{aligned}
& \int_{E} \int_{E} \phi(y) \hat{f}\left(\frac{1}{2} \operatorname{tr}_{E / F}(y z)\right) \mu(z)|z|_{E}^{-s-\frac{1}{2}} d z d y \\
= & \int_{E} \int_{E} \phi(y)\left(\int_{F} f(c) \psi\left(-c \frac{1}{2} \operatorname{tr}_{E / F}(y z)\right) d c\right) \mu(z)|z|_{E}^{-s-\frac{1}{2}} d z d y \\
= & \int_{F} \int_{E} f(c)\left(\int_{E} \phi(y) \psi\left(-c \frac{1}{2} \operatorname{tr}_{E / F}(y z)\right) d y\right) \mu(z)|z|_{E}^{-s-\frac{1}{2}} d z d c \\
= & \int_{F} \int_{E} f(c) \hat{\phi}\left(\frac{1}{2} c z\right) \mu(z)|z|_{E}^{-s-\frac{1}{2}} d z d c \\
= & \mu(2)|2|_{E}^{-s+\frac{1}{2}} \int_{F} f(c) \mu(c)^{-1}|c|_{F}^{2 s-1} d c \int_{E} \hat{\phi}(z) \mu(z)|z|_{E}^{-s-\frac{1}{2}} d z \\
= & \mu(2)|2|_{E}^{-s+\frac{1}{2}} Z_{T}\left(2 s, \mu_{0}^{-1}, f\right) Z_{T}\left(-s+\frac{1}{2}, \mu, \hat{\phi}\right) .
\end{aligned}
$$

Note that we have taken advantage of the fact that $\hat{\phi}(0)=0$ in this manipulation. We find that

$$
\begin{aligned}
Z_{T}\left(-2 s+1, \mu_{0}, \hat{f}\right) \cdot B(s, \mu) \cdot Z_{T}\left(s+\frac{1}{2}, \mu^{-1}, \phi\right) \\
\quad=C \mu(4 \delta)|4 \delta|_{E}^{-s+\frac{1}{2}} \cdot Z_{T}\left(2 s, \mu_{0}^{-1}, f\right) Z_{T}\left(-s+\frac{1}{2}, \mu, \hat{\phi}\right),
\end{aligned}
$$

for the constant $C$ above. Thus,

$$
\begin{aligned}
B(s, \mu) & =C \mu(4 \delta)|4 \delta|_{E}^{-s+\frac{1}{2}} \cdot \frac{Z_{T}\left(2 s, \mu_{0}^{-1}, f\right)}{Z_{T}\left(-2 s+1, \mu_{0}, \hat{f}\right)} \cdot \frac{Z_{T}\left(-s+\frac{1}{2}, \mu, \hat{\phi}\right)}{Z_{T}\left(s+\frac{1}{2}, \mu^{-1}, \phi\right)} \\
& =C \mu(4 \delta)|4 \delta|_{E}^{-s+\frac{1}{2}} \cdot \frac{\rho\left(2 s, \mu_{0}^{-1}, \psi\right)}{\rho\left(s+\frac{1}{2}, \mu^{-1}, \psi_{E}\right)} .
\end{aligned}
$$

Finally, we determine the positive constant $C$, defined by $C \cdot d z=d a d b$, where $d z$ is the self dual measure on $E$ with respect to $\psi_{E}, d a$ and $d b$ are self dual measures on $F$ with respect to $\psi$, and $z=a+2 \delta b$. Take $\phi(a+2 b \delta)=f_{0}(a) f_{1}(b)$ for $f_{0}, f_{1} \in S(F)$. Then, writing $w=x+2 y \delta$, so that

$$
\operatorname{tr}_{E / F}(z w)=2 a x+8 \Delta b y,
$$

we have

$$
\begin{aligned}
C \cdot \hat{\phi}(w) & =\int_{E} \phi(z) \psi_{E}(-z w) C \cdot d z \\
& =\int_{F} \int_{F} f_{0}(a) f_{1}(b) \psi(-(2 a x+8 \Delta b y)) d a d b \\
& =\hat{f}_{0}(2 x) \hat{f}_{1}(8 \Delta y) .
\end{aligned}
$$


Coming back, we have

$$
\begin{aligned}
C^{2} \cdot \phi(z) & =\int_{E} C \cdot \hat{\phi}(w) \psi_{E}(z w) C \cdot d w \\
& =\int_{F} \int_{F} \hat{f}_{0}(2 x) \hat{f}_{1}(8 \Delta y) \psi(2 a x+8 \Delta b y) d x d y \\
& =|2|_{F}^{-4}|\Delta|_{F}^{-1} f_{0}(a) f_{1}(b) \\
& =|2|_{F}^{-4}|\Delta|_{F}^{-1} \phi(z) .
\end{aligned}
$$

Thus

$$
C=|2|_{E}^{-1}|\Delta|_{F}^{-\frac{1}{2}}=|4 \delta|_{E}^{-\frac{1}{2}}
$$

Summarizing, we have:

Corollary 8.3. (i)

$$
M(s, \chi) \Phi_{\eta}=\mu(s, \chi, \eta) \cdot \Phi_{\eta}(-s)
$$

where

$$
\mu(s, \chi, \eta)=\eta(-1) \chi(-\delta)|\delta|_{E}^{s} \frac{\rho\left(2 s, \epsilon_{E / F}, \psi\right)}{\rho\left(s+\frac{1}{2}, \chi \tilde{\eta}^{-1}, \psi_{E}\right)} .
$$

$$
M^{*}(s, \chi) \Phi_{\eta}=\mu^{*}(s, \chi, \eta) \cdot \Phi_{\eta}(-s)
$$

where

Note that

$$
\mu^{*}(s, \chi, \eta)=\eta(-1) \chi(-\delta)|\delta|_{E}^{s} \frac{1}{\rho\left(s+\frac{1}{2}, \chi \tilde{\eta}^{-1}, \psi_{E}\right)} .
$$

$$
\mu^{*}(-s, \check{\chi}, \eta) \mu^{*}(s, \chi, \eta)=1
$$

Proof. We have

$$
\begin{aligned}
M^{*}(s, \chi) \Phi_{\eta}(e) & =\frac{1}{\kappa(s, \chi, \psi)} \mu(s, \chi, \eta) \\
& =\frac{1}{\rho\left(2 s, \chi_{0}, \psi\right)} \eta(-1) \chi(-\delta)|\delta|_{E}^{s} \frac{\rho\left(2 s, \chi_{0}, \psi\right)}{\rho\left(s+\frac{1}{2}, \chi \tilde{\eta}^{-1}, \psi_{E}\right)} \\
& =\eta(-1) \chi(-\delta)|\delta|_{E}^{s} \frac{1}{\rho\left(s+\frac{1}{2}, \chi \tilde{\eta}^{-1}, \psi_{E}\right)}
\end{aligned}
$$

We now return to the calculation of the $\mathrm{L}$ and $\epsilon$ factors defined by doubling.

For a fixed character $\eta$ of $G=E^{1}$, only the section $\Phi_{\bar{\eta}}$ makes a non-zero contribution to the zeta integral. Thus we consider

Proposition 8.4. (i) $\Phi_{\bar{\eta}}(s)$ is a good section, and

$$
Z\left(s, \eta, \Phi_{\bar{\eta}}\right)=1
$$

(ii) $M^{*}(-s, \check{\chi}) \Phi_{\bar{\eta}}$ is a good section, and

$$
Z\left(s, \eta, M^{*}(-s, \check{\chi}) \Phi_{\bar{\eta}}\right)=\mu^{*}(-s, \check{\chi}, \bar{\eta}) .
$$


(iii) The doubling L-factor is

$$
L_{P S R}(s, \eta, \chi)=L(s, \chi \tilde{\eta}) .
$$

(iv) The doubling epsilon factor is:

$$
\epsilon_{P S R}(s, \eta, \chi, \psi)=\eta(-1) \chi(-\delta)|\delta|_{E}^{s-\frac{1}{2}} \epsilon\left(s, \chi \tilde{\eta}, \psi_{E}\right) .
$$

Proof. We compute

$$
\begin{aligned}
Z\left(s, \eta, \Phi_{\bar{\eta}}\right) & =\int_{G} \eta(g) \Phi_{\bar{\eta}}(i(g, 1), s) d g \\
& =\int_{G} \eta(g) \bar{\eta}(g) d g=1 .
\end{aligned}
$$

Then

$$
\begin{aligned}
Z\left(s, \eta, M^{*}(-s, \check{\chi}) \Phi_{\bar{\eta}}\right) & =\mu^{*}(-s, \check{\chi}, \bar{\eta}) Z\left(s, \eta, \Phi_{\bar{\eta}}\right) \\
& =\mu^{*}(-s, \check{\chi}, \bar{\eta}) .
\end{aligned}
$$

Since

$$
\begin{aligned}
\mu^{*}(-s, \check{\chi}, \bar{\eta}) & =\eta(-1) \check{\chi}(-\delta)|\delta|_{E}^{-s} \frac{1}{\rho\left(-s+\frac{1}{2}, \check{\chi} \tilde{\eta}, \psi_{E}\right)} \\
& =L\left(s+\frac{1}{2}, \check{\chi}^{-1} \tilde{\eta}^{-1}\right) \cdot(\text { entire })
\end{aligned}
$$

we see that the doubling L-factor is $L\left(s, \check{\chi}^{-1} \tilde{\eta}^{-1}\right)$. Note that, if $\lambda$ is any character of $E^{\times}$, then, setting $\lambda^{\prime}(x)=\lambda(\bar{x})$, we have $L\left(s, \lambda^{\prime}\right)=L(s, \lambda)$. Also note that $\check{\chi}^{-1}=\chi^{\prime}$ and $\tilde{\eta}^{-1}=\tilde{\eta}^{\prime}$. Thus the doubling L-factor is as claimed.

Finally, we consider the functional equation:

$$
Z\left(-s, \eta, M^{*}(s, \chi) \Phi_{\bar{\eta}}\right)=\mu^{*}(s, \chi, \bar{\eta}),
$$

so that

$$
\Gamma^{*}(s, \chi, \eta, \psi)=\mu^{*}(s, \chi, \bar{\eta})
$$

Hence

$$
\begin{aligned}
\epsilon_{P S R}\left(s+\frac{1}{2}, \eta, \chi, \psi\right) & =\mu^{*}(s, \chi, \bar{\eta}) \cdot \frac{L\left(s+\frac{1}{2}, \chi \tilde{\eta}\right)}{L\left(-s+\frac{1}{2}, \check{\chi} \tilde{\eta}\right)} \\
& =\eta(-1) \chi(-\delta)|\delta|_{E}^{s} \frac{1}{\rho\left(s+\frac{1}{2}, \chi \tilde{\eta}, \psi_{E}\right)} \cdot \frac{L\left(s+\frac{1}{2}, \chi \tilde{\eta}\right)}{L\left(-s+\frac{1}{2}, \tilde{\chi} \tilde{\eta}\right)} \\
& =\eta(-1) \chi(-\delta)|\delta|_{E}^{s} \epsilon\left(s+\frac{1}{2}, \chi \tilde{\eta}, \psi_{E}\right) .
\end{aligned}
$$

Combining (iv) of Proposition 8.4 with Theorem 6.1, we obtain

Corollary 8.5. Let $W$ (resp. $V$ ) be a one dimensional skew-Hermitian (resp. Hermitian) space. Suppose that the skew-Hermitian form on $W=E$ is given by $\langle x, y\rangle=a \delta x \bar{y}$, and that the Hermitian form on $V=E$ is given by $(x, y)=b \bar{x} y$ for $a$ and $b \in F^{\times}$. Let

$$
\epsilon(V)=\epsilon_{E / F}(b), \quad \text { and } \quad \epsilon(W)=\epsilon_{E / F}(a) .
$$


Fix a character $\chi$ of $E^{\times}$whose restriction to $F^{\times}$is $\epsilon_{E / F}$. Let $\omega_{V, \chi, \psi}$ be the Weil representation $\omega_{V, \chi, \psi}=\omega_{\psi} \circ \tilde{\iota}_{V, \chi}$ of $G(W)=E^{1}$ obtained via the homomorphism

$$
\tilde{\iota}_{V, \chi, \psi}: E^{1}=G(W) \longrightarrow M p(\mathbb{W})
$$

of (1.17). Then

$$
\omega_{V, \chi, \psi}=\bigoplus_{\eta} \mathbb{C}_{\eta},
$$

where $\eta$ runs over all characters of $E^{1}$ such that

$$
\epsilon\left(\frac{1}{2}, \chi \tilde{\eta}^{-1}, \psi_{E}\right) \cdot \eta(-1) \cdot \chi(-\delta) \cdot \epsilon_{E / F}(-2)=\epsilon(V) \epsilon(W) .
$$

Here $\tilde{\eta}(x)=\eta\left(\frac{x}{\bar{x}}\right)$ is the (standard) base change of $\eta$ to $G L_{1}(E)$.

Remark. This result is due to Rogawski [42], whose proof was quite different and, in particular, relied on some global arguments and some calculations involving Gauss sums. It has also been verified by direct computation by Tonghai Yang [52].

\section{APPENDIX}

In this section we record several useful properties of the splitting.

Suppose that $W$ is a split skew-Hermitian space, and let $\Omega(W)$ be the space of maximal isotropic subspaces of $W$. For a Hermitian space $V$, and $\mathbb{W}=V \otimes_{E} W$, with symplectic form

$$
\langle\langle,\rangle\rangle=\frac{1}{2} \operatorname{tr}_{E / F}((,) \otimes \overline{\langle,\rangle})
$$

let

$$
\iota_{V}: G(W) \longrightarrow S p(\mathbb{W}),
$$

be the associated symplectic embedding. If $\operatorname{dim}_{E} V=m$, let $\chi$ be a character of $E^{\times}$such that $\left.\chi\right|_{F^{\times}}=\epsilon_{E / F}^{m}$. Then, associated to $\chi$ and to a choice of $Y \in \Omega(W)$, we have the splitting homomorphism

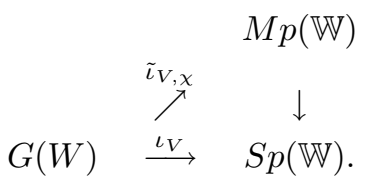

Explicitly, the maximal isotropic subspace $\mathbb{Y}=V \otimes Y$ of $\mathbb{W}$, associated to $Y$, determines an isomorphism of sets

$$
M p(\mathbb{W}) \simeq S p(\mathbb{W}) \times \mathbb{C}^{1} .
$$

The splitting homomorphism is given by

$$
\tilde{\iota}_{V, \chi}(g)=\left(\iota_{V}(g), \beta_{V, \chi}(g)\right),
$$

where

$$
\beta_{V, \chi}(g)=\chi(x(g)) \gamma_{F}(\eta \circ R V)^{-j(g)},
$$

with $x(g), j(g)$, and $R V$ given in [22].

Our first result is the following.

Proposition A.1. The splitting homomorphism $\tilde{\iota}_{V, \chi}$ is independent of the choice of $Y \in \Omega(W)$, and thus depends only on $\chi$. 
Proof. To begin, for any pair of maximal isotropic subspaces $\mathbb{Y}_{1}$ and $\mathbb{Y}_{2}$ of $\mathbb{W}$, we consider the diagram:

$$
\begin{array}{cccc}
M p(\mathbb{W}) & \stackrel{\sim}{\longrightarrow} & S p(\mathbb{W}) \times \mathbb{C}^{1} & (g, z) \\
\| & & \downarrow & \downarrow \\
M p(\mathbb{W}) & \stackrel{\sim}{\longrightarrow} & S p(\mathbb{W}) \times \mathbb{C}^{1} & (g, z \lambda(g)),
\end{array}
$$

where the isomorphism in the first row is determined by $\mathbb{Y}_{1}$, and the isomorphism in the second row is determined by $\mathbb{Y}_{2}$. According to Lemma 4.2 of [22], the factor $\lambda(g)$ is given by

$$
\begin{aligned}
\lambda(g) & =\gamma_{F}\left(\eta \circ L\left(\mathbb{Y}_{1}, \mathbb{Y}_{2} g^{-1}, \mathbb{Y}_{2}\right)\right) \gamma_{F}\left(\eta \circ L\left(\mathbb{Y}_{1}, \mathbb{Y}_{2}, \mathbb{Y}_{1} g\right)\right) \\
& =c_{\mathbb{Y}_{1}}\left(\alpha, g \alpha^{-1}\right) c_{\mathbb{Y}_{1}}\left(g, \alpha^{-1}\right),
\end{aligned}
$$

where $\mathbb{Y}_{2}=\mathbb{Y}_{1} \alpha$, for $\alpha \in S p(\mathbb{W})$. Now assume that $\mathbb{Y}_{1}=V \otimes Y_{1}$ and $\mathbb{Y}_{2}=V \otimes Y_{2}$, for $Y_{1}$ and $Y_{2} \in \Omega(W)$. We may assume that $\alpha \in G(W)$ (which we temporarily identify with its image under $\iota_{V}$ ) with $Y_{2}=Y_{1} \alpha$. Then,

$$
c_{\mathbb{Y}_{1}}\left(\alpha, g \alpha^{-1}\right)=\beta_{V, 1}\left(\alpha g \alpha^{-1}\right) \beta_{V, 1}(\alpha)^{-1} \beta_{V, 1}\left(g \alpha^{-1}\right)^{-1},
$$

and

$$
c_{\mathbb{Y}_{1}}(g, \alpha)=\beta_{V, 1}\left(g \alpha^{-1}\right) \beta_{V, 1}(g)^{-1} \beta_{V, 1}\left(\alpha^{-1}\right)^{-1},
$$

where $\beta_{V, 1}(g)$ is the function on $G(W)$ which defines the splitting homomorphism associated to $Y_{1}$. Thus

$$
\lambda(g)=\beta_{V, 1}\left(\alpha g \alpha^{-1}\right)\left[\beta_{V, 1}(\alpha) \beta_{V, 1}\left(\alpha^{-1}\right) \beta_{V, 1}(g)\right]^{-1} .
$$

Now we observe that, if $g \in G(W)$, then for any $Y \in \Omega(W)$ with associated functions $j$ and $x$,

$$
j\left(g^{-1}\right)=j(g), \quad \text { and } \quad x\left(g^{-1}\right)=(-1)^{j(g)} x(g)^{-1} .
$$

Thus, we let $j_{1}(g), x_{1}(g)$ and $\beta_{V, 1}(g)$ be the quantities associated to $Y_{1}$,

$$
\begin{aligned}
\beta_{V, 1}\left(g^{-1}\right) & =\chi\left(x_{1}\left(g^{-1}\right)\right) \gamma_{F}(\eta \circ R V)^{-j_{1}\left(g^{-1}\right)} \\
& =\chi(-1)^{j_{1}(g)} \chi\left(x_{1}(g)\right)^{-1} \gamma_{F}(\eta \circ R V)^{-j_{1}(g)} .
\end{aligned}
$$

Hence, since

$$
\gamma_{F}(\eta \circ R V)=(\Delta, \operatorname{det}(V))_{F} \gamma_{F}(-\Delta, \eta)^{m} \gamma_{F}(-1, \eta)^{-m},
$$

we have

$$
\beta_{V, 1}(\alpha) \beta_{V, 1}\left(\alpha^{-1}\right)=\chi\left(x_{1}(\alpha)\right) \chi\left(x_{1}\left(\alpha^{-1}\right)\right) \gamma_{F}(\eta \circ R V)^{-j_{1}(\alpha)} \gamma_{F}(\eta \circ R V)^{-j_{1}\left(\alpha^{-1}\right)}
$$

$$
\begin{aligned}
& =\chi(-1)^{j_{1}(\alpha)} \gamma_{F}(\eta \circ R V)^{-2 j_{1}(\alpha)} \\
& =\epsilon_{E / F}(-1)^{m j_{1}(\alpha)}(-1,-\Delta)_{F}^{m j_{1}(\alpha)}(-1,-1)_{F}^{m j_{1}(\alpha)} \\
& =1 .
\end{aligned}
$$

Thus

$$
\lambda(g)=\beta_{V, 1}\left(\alpha g \alpha^{-1}\right) \beta_{V, 1}(g)^{-1} .
$$

On the other hand, we let $j_{2}(g), x_{2}(g)$, and $\beta_{V, 2}(g)$ be the quantities associated to $Y_{2}$. Let $P_{1}$ (resp. $P_{2}$ ) be the stabilizer of $Y_{1}$ (resp. $Y_{2}$ ). Then $P_{2}=\alpha^{-1} P_{1} \alpha$, and 
$\tau_{S, 2}=\alpha^{-1} \tau_{S, 1} \alpha$, where the $\tau_{S, i}, i=1,2$ are the Weyl elements for $P_{i}$, as in [40] and [22]. Thus,

$$
g=p_{2} \tau_{S, 2} p_{2}^{\prime} \quad \Longrightarrow \quad \alpha g \alpha^{-1}=\alpha p_{2} \alpha^{-1} \tau_{S, 1} \alpha p_{2}^{\prime} \alpha^{-1},
$$

so that

$$
x_{1}\left(\alpha g \alpha^{-1}\right)=x_{2}(g), \quad \text { and } \quad j_{1}\left(\alpha g \alpha^{-1}\right)=j_{2}(g),
$$

and so

$$
\beta_{V, 1}\left(\alpha g \alpha^{-1}\right)=\beta_{V, 2}(g) .
$$

Therefore,

$$
\lambda(g)=\beta_{V, 2}(g) \beta_{V, 1}(g)^{-1},
$$

as required.

Corollary A.2. Suppose that $V$ and $\chi$ are fixed, as above. If $W$ is a split space and $W=W_{1}+W_{2}$, with $W_{1}$ and $W_{2}$ also split, then the following diagram commutes:

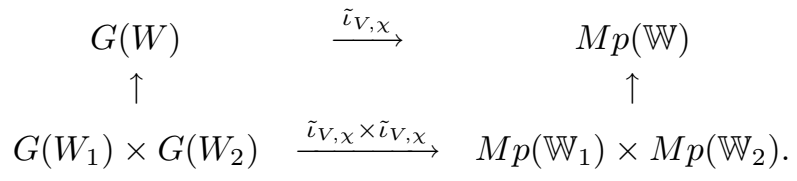

In particular, the restriction of the splitting $\tilde{\iota}_{V, \chi}$ of $G(W)$ to any $G\left(W_{1}\right)$ for a split subspace $W_{1}$ in $W$ is the corresponding splitting, determined by $\chi$, for $G\left(W_{1}\right)$.

Proof. By Proposition 1, we can work with a maximal isotropic subspace of $W$ of the form $Y=Y_{1}+Y_{2}$, for $Y_{i} \in \Omega\left(W_{i}\right)$. If $x, j, x_{1}, j_{1}, x_{2}$, and $j_{2}$ are the associated functions on $G(W), G\left(W_{1}\right)$ and $G\left(W_{2}\right)$ respectively, then, for $g=\left(g_{1}, g_{2}\right)$, we clearly have

$$
x(g)=x\left(g_{1}\right) x\left(g_{2}\right) \quad \text { and } \quad j(g)=j\left(g_{1}\right)+j\left(g_{2}\right) .
$$

This implies that

$$
\beta_{V}(g)=\beta_{V}\left(g_{1}\right) \beta_{V}\left(g_{2}\right),
$$

as claimed.

Next, for $W$ an arbitrary skew-Hermitian space, we define $\tilde{\iota}_{V, \chi}$ by the doubling construction of section 4 of [22]. Thus, we let $W_{-}$be the space $W$ with the negative of the given form, let

$$
\widetilde{W}=W+W_{-},
$$

and let

$$
i: G(W) \longrightarrow G(\widetilde{W})
$$

be the embedding defined by letting $G(W)$ act in the natural way on $W$ and trivially on $W_{-}$. We define the splitting $\tilde{\iota}_{V, \chi}$ so that the diagram

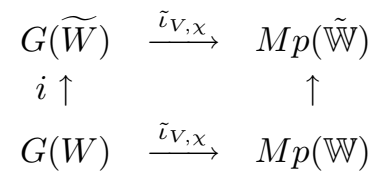

commutes. Note that, if $W$ is itself split, we have a potential conflicting notation. But, by Corollary A.2, applied to $\widetilde{W} \supset W$, we find that the two definitions coincide! 
Corollary A.3. Suppose that $W$ is any skew-Hermitian space, and that $W=W_{1}+$ $W_{2}$, for skew-Hermitian spaces $W_{1}$ and $W_{2}$. For $V$ and $\chi$ as above, let $\tilde{\iota}_{V, \chi}$ be the splitting homomorphism for each of $G(W), G\left(W_{1}\right)$ and $G\left(W_{2}\right)$, determined by doubling. Then the following diagram commutes:

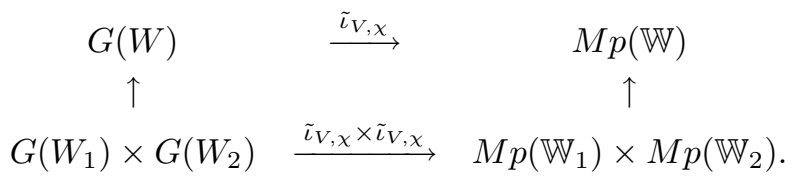

In particular, the splitting $\tilde{\iota}_{V, \chi}$ determined by $\chi$ is compatible with restriction to $G\left(W_{1}\right) \subset G(W)$, where $W_{1} \subset W$ is any non-degenerate subspace.

Proof. Consider the diagram

$$
\begin{array}{clc}
M p\left(\tilde{\mathbb{W}}_{1}\right) \times M p\left(\tilde{\mathbb{W}}_{2}\right) & \longrightarrow & M p(\tilde{\mathbb{W}}) \\
\tilde{\iota}_{V, \chi} \uparrow & & \tilde{\iota}_{V, \chi} \uparrow \\
G\left(\widetilde{W}_{1}\right) \times G\left(\widetilde{W}_{2}\right) & \longrightarrow & G(\widetilde{W}) \\
i \times i \uparrow & & i \uparrow \\
G\left(W_{1}\right) \times G\left(W_{2}\right) & \longrightarrow & G(W),
\end{array}
$$

obtained by doubling the spaces $W, W_{1}$ and $W_{2}$. The bottom square clearly commutes while the top square commutes by Corollary A.2.

This result should be very useful in applications!

We record here several other technical results. In particular, we determine the dependence of our splittings on scaling of the form on $W$.

First we change notation for a moment and let $W,\langle$,$\rangle be a symplectic vector$ space over $F$ of dimension $2 n$. For $\alpha \in F^{\times}$, let $W_{\alpha}$ be the space $W$ with symplectic form $\alpha \cdot\langle$,$\rangle . Then any maximal isotropic subspace Y$ in $W$ is also an isotropic subspace $Y_{\alpha}$ in $W_{\alpha}$, and we then obtain a diagram:

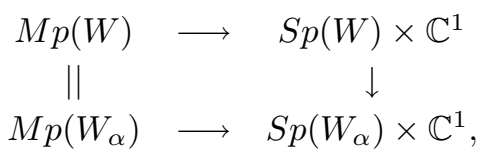

of 'Rao' isomorphisms.

Proposition A.4. The right hand arrow in the previous diagram is given by

$$
(g, z) \mapsto\left(g, z \cdot(\alpha, x(g))_{F} \gamma_{F}(\alpha, \eta)^{-j(g)}\right) .
$$

Proof. We need to compare the two Rao cocycles:

$$
c_{Y}\left(g_{1}, g_{2}\right)=\gamma_{F}\left(\eta \circ L\left(Y, Y g_{2}^{-1}, Y g_{1}\right)\right)
$$

and

$$
c_{Y_{\alpha}}\left(g_{1}, g_{2}\right)=\gamma_{F}\left(\eta \circ L\left(Y_{\alpha}, Y_{\alpha} g_{2}^{-1}, Y_{\alpha} g_{1}\right)\right) .
$$

Since

$$
L\left(Y_{\alpha}, Y_{\alpha} g_{2}^{-1}, Y_{\alpha} g_{1}\right)=\alpha \cdot L\left(Y, Y g_{2}^{-1}, Y g_{1}\right)=\alpha \cdot L,
$$


we compute:

$$
\begin{aligned}
\gamma_{F}(\eta \circ \alpha \cdot L)= & \gamma_{F}(\operatorname{det}(\alpha L), \eta) \gamma_{F}(\eta)^{\ell} h_{F}(\alpha L) \\
= & (\alpha, \operatorname{det} L)_{F}^{\ell} \gamma_{F}(\eta)^{\ell}(\alpha,-1)_{F}^{\frac{\ell(\ell-1)}{2}}(\alpha, \operatorname{det} L)_{F}^{\ell-1} h_{F}(\ell) \\
= & (\alpha, \operatorname{det} L)_{F} \gamma_{F}(\alpha, \eta)^{\ell} \gamma_{F}(\eta \circ L) \\
= & (\alpha,-1)^{t}\left(\alpha, x\left(g_{1} g_{2}\right)\right)_{F}\left(\alpha, x\left(g_{1}\right)\right)_{F}\left(\alpha, x\left(g_{2}\right)\right)_{F} \\
& \quad \times \gamma_{F}(\alpha, \eta)^{j_{1}+j_{2}-j}(-1, \alpha)^{t} \gamma_{F}(\eta \circ L),
\end{aligned}
$$

where we have used the identities,

$$
h_{F}(\alpha L)=(\alpha,-1)_{F}^{\frac{\ell(\ell-1)}{2}}(\alpha, \operatorname{det} L)_{F}^{\ell-1} h_{F}(\ell),
$$

for the Hasse invariant, and

$$
\operatorname{det}(L)=(-1)^{t} x\left(g_{1} g_{2}\right) x\left(g_{1}\right) x\left(g_{2}\right),
$$

and

$$
\ell=\operatorname{dim}_{F} L=j_{1}+j_{2}-j-2 t,
$$

from [22]. This quantity is clearly the coboundary of the function

$$
g \mapsto(\alpha, x(g))_{F} \gamma_{F}(\alpha, \eta)^{-j(g)},
$$

as required.

We now return to our previous notation, and, for $\alpha \in F^{\times}$, we consider $W$ and the scaled space $W_{\alpha}$. We then obtain the diagram

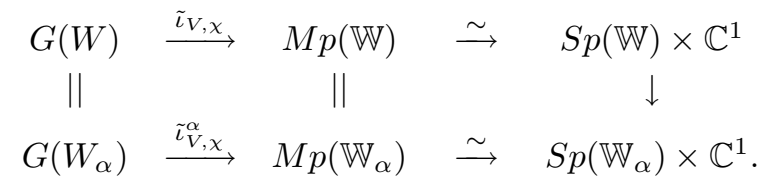

We want to determine the factor by which it fails to commute. Going around the top of the diagram, we find that $g \in G(W)$ goes to

$$
\left(\iota_{V}(g), \beta_{V, \chi}(g) \cdot\left(\alpha, x\left(\iota_{V}(g)\right)\right)_{F} \gamma_{F}(\alpha, \eta)^{-j\left(\iota_{V}(g)\right)}\right) .
$$

Lemma A.5. (i) $x\left(\iota_{V}(g)\right)=N_{E / F}(x(g))^{m} \cdot(-\Delta)^{m j(g)}$.

(ii) $j\left(\iota_{V}(g)\right)=2 m j(g)$.

Thus, the $\mathbb{C}^{1}$ term above becomes

$$
\begin{aligned}
& \beta_{V, \chi}(g)\left(\alpha, N_{E / F}(x(g))\right)^{m}(\alpha,-\Delta)_{F}^{m j(g)}(-1, \alpha)_{F}^{m j(g)} \\
= & \beta_{V, \chi}(g)\left(\alpha, N_{E / F}(x(g))\right)^{m} \chi(\alpha)^{j(g)} .
\end{aligned}
$$

On the other hand, when the form on $W$ is scaled by $\alpha$, the standard symplectic basis $e_{1}, \ldots, e_{n}^{\prime}$, which we write as $e, e^{\prime}$ for short, must be changed to, say, $e, \alpha^{-1} e^{\prime}$, and thus Rao's Weyl element $\tau$, which is defined by $\tau e=-e^{\prime}$ and $\tau e^{\prime}=e$, must be modified:

$$
\tau_{\alpha}=\tau\left(\begin{array}{ll}
\alpha & \\
& \alpha^{-1}
\end{array}\right)
$$

Thus,

$$
g=p_{1} \tau_{S, \alpha} p_{2}=p_{1} \tau_{S}\left(\begin{array}{cc}
\alpha_{S} & \\
& \alpha_{S}^{-1}
\end{array}\right) p_{2}
$$


so that

$$
x_{\alpha}(g)=\alpha^{j(g)} x(g)
$$

and $j_{\alpha}(g)=j(g)$, where $x_{\alpha}$ and $j_{\alpha}$ are the Rao functions for $W_{\alpha}$. Using these quantities in $\beta_{V, \alpha, \chi}(g)$, we find that the image of $g$ around the bottom part of the diagram is:

$$
\left(\iota_{V}(g), \beta_{V, \alpha, \chi}(g)\right)
$$

with

$$
\beta_{V, \alpha, \chi}(g)=\chi\left(x_{\alpha}(g)\right) \gamma_{F}(\eta \circ R V)^{-j_{\alpha}(g)}=\chi(\alpha)^{j(g)} \beta_{V, \chi}(g) .
$$

Comparing these two results, we conclude the following:

Proposition A.6. Suppose that $V$ and $\chi$ are fixed, and that, for $\alpha \in F^{\times}, W_{\alpha}$ is the skew-Hermitian space obtained by scaling the form on $W$ by $\alpha$. Then the splittings

$$
\tilde{\iota}_{V, \chi}, \tilde{\iota}_{V, \alpha, \chi}: G(W)=G\left(W_{\alpha}\right) \longrightarrow M p(\mathbb{W})=M p\left(\mathbb{W}_{\alpha}\right)
$$

differ by a quadratic character:

$$
\tilde{\iota}_{V, \alpha, \chi}(g)=\eta_{\alpha}(g)^{m} \cdot \tilde{\iota}_{V, \chi}(g),
$$

where

$$
\eta_{\alpha}(g)=\left(\alpha, N_{E / F}(x(g))\right)_{F} .
$$

Note that the twisting character $\eta_{\alpha}^{m}$ is trivial if $m=\operatorname{dim}_{E}(V)$ is even.

Since the identity

$$
\operatorname{det}(g)=\left(\frac{x(g)}{\overline{x(g)}}\right)
$$

determines $x(g) \in E^{\times}$up to multiplication by an element $\alpha$ of $F^{\times}$, the map

$$
\eta_{\alpha}(g)=\left(\alpha, N_{E / F}(x(g))\right)_{F}
$$

indeed defines a quadratic character $\eta_{\alpha}$ of $G(W)$.

Now $U=V \otimes W$ is a skew-Hermitian space over $E$ with inner product:

$$
\left\langle v_{1} \otimes w_{1}, v_{2} \otimes w_{2}\right\rangle=\left(v_{2}, v_{1}\right)\left\langle w_{1}, w_{2}\right\rangle .
$$

Here $U$ is a left vector space over $E$, where $\alpha \cdot v \otimes w=v \otimes \alpha w$. Let $V_{1}=E$ with Hermitian form $(x, y)_{1}=\bar{x} y$, and note that

$$
\mathbb{W}=R_{E / F}\left(V \otimes_{E} W\right) \simeq R_{E / F}\left(V_{1} \otimes_{E} U\right) .
$$

If $\chi_{1}$ is a character of $E^{\times}$with $\left.\chi_{1}\right|_{F^{\times}}=\epsilon_{E / F}$, there is a splitting

$$
G(U) \stackrel{\tilde{\iota}_{V_{1}, \chi_{1}}}{\longrightarrow} M p(\mathbb{W})
$$

as usual. Let

$$
G(V) \times G(W) \stackrel{i}{\rightarrow} G(U)
$$

be the natural embedding.

Lemma A.7. For $m=\operatorname{dim}_{E} V$, set $\chi_{m}=\chi_{1}^{m}$. Then on $G(W)$,

$$
\tilde{\iota}_{V_{1}, \chi_{1}} \circ i=\tilde{\iota}_{V, \chi_{m}} \text {. }
$$


Proof. First we assume that $W$ and hence $U$ is split, and choose a polarization $W=X+Y$ and hence $U=V \otimes_{E} X+V \otimes_{E} Y$. Then

$$
\mathbb{Y}=R_{E / F}\left(V \otimes_{E} Y\right) \simeq R_{E / F}\left(V_{1} \otimes_{E}\left(V \otimes_{E} Y\right)\right)
$$

and we use the isomorphism $M p(\mathbb{W}) \simeq S p(\mathbb{W}) \times \mathbb{C}^{1}$. If we let $x_{U}$ and $j_{U}$ be the Rao functions for $G(U)$, then for $g \in G(W)$,

$$
j_{U}(i(g))=m j(g), \quad \text { and } \quad x_{U}(i(g))=x(g)^{m} \cdot(\operatorname{det} V)^{j(g)} .
$$

Thus

$$
\begin{aligned}
\beta_{V_{1}, \chi_{1}}(i(g)) & =\chi_{1}\left(x_{U}(i(g))\right) \cdot \gamma_{F}\left(\eta \circ R V_{1}\right)^{-j_{U}(i(g))} \\
& =\chi_{1}(x(g))^{m} \chi_{1}(\operatorname{det} V)^{j(g)} \gamma_{F}(-\Delta, \eta)^{-m j(g)} \gamma_{F}(-1, \eta)^{m j(g)} \\
& =\chi_{m}(x(g))\left((\Delta, \operatorname{det} V)_{F} \gamma_{F}(-\Delta, \eta)^{m} \gamma_{F}(-1, \eta)^{-m}\right)^{-j(g)} \\
& =\chi_{m}(x(g)) \gamma_{F}(\eta \circ R V)^{-j(g)} \\
& =\beta_{V, \chi_{m}}(g),
\end{aligned}
$$

as claimed.

Next, if $W$ is arbitrary, we double both $W$ and $U$ and obtain the claimed relation from that just proved for the split case.

Now we recall the 'reflection principle' used at the end of section 1 to construct a splitting over $G(V)$. As in section 1 , let $W^{\prime}=W$ with Hermitian form

$$
\left(w_{1}^{\prime}, w_{2}^{\prime}\right)^{\prime}=\delta^{-1}\left\langle w_{2}, w_{1}\right\rangle,
$$

and let $V^{\prime}=V$ with skew-Hermitian form

$$
\left\langle v_{1}^{\prime}, v_{2}^{\prime}\right\rangle^{\prime}=\delta\left(v_{2}, v_{1}\right)
$$

Here, for $w \in W$ (resp. $v \in V$ ), we let $w^{\prime}$ (resp. $v^{\prime}$ ) denote the corresponding element of $W^{\prime}\left(\right.$ resp. $\left.V^{\prime}\right)$. Let $U^{\prime}=W^{\prime} \otimes_{E} V^{\prime}$ with

$$
\begin{aligned}
\left\langle w_{1}^{\prime} \otimes v_{1}^{\prime}, w_{2}^{\prime} \otimes v_{2}^{\prime}\right\rangle^{\prime} & =\left(w_{2}^{\prime}, w_{1}^{\prime}\right)^{\prime}\left\langle v_{1}^{\prime}, v_{2}^{\prime}\right\rangle^{\prime} \\
& =\left\langle w_{1}, w_{2}\right\rangle\left(v_{2}, v_{1}\right) \\
& =\left\langle v_{1} \otimes w_{1}, v_{2} \otimes w_{2}\right\rangle .
\end{aligned}
$$

Thus, the map $\alpha: U^{\prime} \longrightarrow U$ given by $w^{\prime} \otimes v^{\prime} \mapsto v \otimes w$ is an isometry of skewHermitian spaces. We then have the commutative diagram:

$$
\begin{array}{ccccr}
G(V) \times G(W) & \longrightarrow & G(U) & \stackrel{\tilde{\iota}_{V_{1}, \chi_{1}}}{\longrightarrow} & M p(\mathbb{W}) \\
s w \downarrow & & A d(\alpha) \downarrow & & \downarrow \widehat{A d(\alpha)} \\
G\left(W^{\prime}\right) \times G\left(V^{\prime}\right) & \longrightarrow & G\left(U^{\prime}\right) & \stackrel{\tilde{\iota}_{V_{1}, \chi_{1}}}{\longrightarrow} & M p\left(\mathbb{W}^{\prime}\right),
\end{array}
$$

where the left most arrow just switches factors. Now, by definition,

$$
\tilde{\iota}_{W, \chi^{\prime}}: G(V) \longrightarrow M p(\mathbb{W})
$$

is given by

$$
\tilde{\iota}_{W, \chi^{\prime}}=\widetilde{\operatorname{Ad}(\alpha)}^{-1} \circ \tilde{\iota}_{W^{\prime}, \chi^{\prime}},
$$

and, by the previous result,

$$
\tilde{\iota}_{W, \chi_{n}}(h)=\tilde{\iota}_{V_{1}, \chi_{1}}(i(h)) .
$$


Corollary A.8. If $\chi_{1}$ is a character of $E^{\times}$with $\left.\chi_{1}\right|_{F^{\times}}=\epsilon_{E / F}$, and if $\chi_{m}=\chi_{1}^{m}$ and $\chi_{n}=\chi_{1}^{n}$, then for $z \in E^{1}$,

$$
\tilde{\iota}_{V, \chi_{m}}\left(z \cdot 1_{W}\right)=\tilde{\iota}_{W, \chi_{n}}\left(z \cdot 1_{V}\right)
$$

i.e., if the pair of characters $\left(\chi_{m}, \chi_{n}\right)$ is used to define the splitting over $G(V) \times$ $G(W)$, then the splittings agree on the centers of $G(V)$ and $G(W)$. In particular, if irreducible representations $\pi$ of $G(W)$ and $\tau$ of $G(V)$ correspond, i.e., if $\tau=$ $\theta_{\chi}(\pi, V)$, then they have the same central character.

Corollary A.9. If $m=n=1$ and if the same character $\chi$ is used to define the splitting for $G(V)$ and $G(W)$, then the theta correspondence is the identity map, $\eta \leftrightarrow \eta$, on those representations which occur.

Finally, suppose that $W^{\prime} \simeq V$, and hence $V^{\prime} \simeq W$. Then we may identify the spaces $U$ and $U^{\prime}$, and view $\alpha: U \longrightarrow U^{\prime}=U$ as an element of $G(U)$. We then have the commutative diagram:

$$
\begin{array}{cccll}
G(V) \times G(W) & \longrightarrow & G(U) & \stackrel{\tilde{\iota}_{V_{1}, \chi_{1}}}{\longrightarrow} & M p(\mathbb{W}) \\
s w \downarrow & & A d(\alpha) \downarrow & & \\
G\left(W^{\prime}\right) \times G\left(V^{\prime}\right) & \longrightarrow & G(U) & \stackrel{\tilde{\iota}_{V_{1}, \chi_{1}}}{\longrightarrow} &
\end{array}
$$

Corollary A.10. If $W^{\prime} \simeq V$ and if the same character $\chi$ is used to define the splittings over $G(V)$ and $G(W)$, then the Weil representation $\omega_{V, W, \chi}$ of $G(V) \times$ $G(W)$ is isomorphic to the representation $\omega_{W^{\prime}, V^{\prime}, \chi}$ of $G\left(W^{\prime}\right) \times G\left(V^{\prime}\right) \simeq G(V) \times$ $G(W)$.

Note that both the definition of the splitting over $G(V)$ (resp. $\left.G\left(W^{\prime}\right)\right)$ and the definition of $W^{\prime}$ (resp. $V^{\prime}$ ) depend on the choice of $\delta$.

\section{REFERENCES}

1. J. Adams, L-functoriality for dual pairs, Astérisque 171-172 (1989), 85-129. MR 91e:55016

2. J. Arthur, On some problems suggested by the trace formula, Proc. of the Special Year in Harmonic Analysis, University of Maryland, Lecture Notes in Math. 1024, Springer-Verlag, New York, 1984. MR 85k:11025

3. J. Arthur, Unipotent automorphic representations: conjectures, Orbites unipotentes et representations, II, Astérisque 171-172, 1989, pp. 13-73. MR 91f:22030

4. S. Brocco, On the non-vanishing of theta-liftings from $U(1)$ to $U(1)$, Ph.D. Thesis, Brandeis University (1992).

5. L. Clozel, Représentations Galoisiennes associées aux représentations automorphes autoduales de $G L(n)$, Publ. Math. IHES 73 (1991), 97-145. MR 92i:11055

6. S. Gelbart and J. Rogawski, L-functions and Fourier-Jacobi coefficients for the unitary group U(3), Invent. Math. 105 (1991), 445-472. MR 93b:11059

7. S. Gelbart and J. Rogawski, Exceptional representations and Shimura's integral for the local unitary group U(3), Festschrift in Honor of Piatetski-Shapiro, vol. 2, Israel Math. Conf. Proc., 1990, pp. 19-75. MR 93h:22029

8. S. Gelbart, J. Rogawski and D. Soudry, On the genericity of endoscopic L-packets on U(3), preprint.

9. S. Gelbart, J. Rogawski and D. Soudry, On periods of cusp forms and algebraic cycles for U(3), Israel J. Math. 83 (1993), 213-252. MR 95a:11047

10. S. Gelbart, J. Rogawski and D. Soudry, Periods of cusp forms and L-packets, C. R. Acad. Sci. Paris Sér. I Math. 317 (1993), 717-722. MR 94m:11064

11. R. Godement and H. Jacquet, Zeta functions of simple algebras, Lecture Notes in Math. 260, Springer, New York, 1972. MR 49:7241

12. B. H. Gross, L-functions at the central critical point, Motives, part I, Proc. Symp. Pure Math., vol. 55, AMS, 1994, pp. 527-535. MR 95a:11060 
13. B. H. Gross and S. S. Kudla, Heights and the central critical values of triple product Lfunctions, Compositio Math. 81 (1992), 143-209. MR 93g:11047

14. B. H. Gross and D. Prasad, On the decomposition of a representation of $\mathrm{SO}_{n}$ when restricted to $S O_{n-1}$, Canadian J. Math. 44 (1992), 974-1002. MR 93j:22031

15. M. Harris, L-functions of $2 \times 2$ unitary groups and factorization of periods of Hilbert modular forms, Jour. AMS 6 (1993), 637-719. MR 93m:11043

16. M. Harris, Non-vanishing of L-functions of $2 \times 2$ unitary groups, Forum Math. 5 (1993), 405-419. MR 94k:11057

17. M. Harris and S. Kudla, The central critical value of a triple product L-function, Annals of Math. 133 (1991), 605-672. MR 93a:11043

18. R. Howe, $L^{2}$ duality for stable reductive dual pairs.

19. H. Jacquet, Principal L-functions of the linear group, Automorphic Forms, Representations and $L$-Functions, part 2, Proc. Symp. Pure Math., vol. 33, AMS, Providence, 1979, pp. 63-86. MR 81f:22029

20. M. Karel, Values of certain Whittaker functions on p-adic groups, Ill. Jour. of Math. 26 (1983), 552-575. MR 84f: 10033

21. S. Kudla, On the local theta correspondence, Inventiones math. 83 (1986), 229-255. MR 87e:22037

22. S. Kudla, Splitting metaplectic covers of dual reductive pairs, Israel J. Math. 87 (1994), 361401. MR 95h:22019

23. S. Kudla and S. Rallis, A regularized Siegel-Weil formula, the first term identity, Annals of Math. 140 (1994), 1-80. MR 95f:11036

24. S. Kudla and S. Rallis, On first occurrence in the local theta correspondence, preprint, 1996.

25. S. Kudla and W. Sweet, Degenerate principal series for unitary groups, preprint (1994).

26. J.-P. Labesse and R. P. Langlands, L-indistinguishability for $S L(2)$, Canad. J. Math. 31 (1979), 726-85. MR 81b:22017

27. R. P. Langlands and D. Ramakrishnan, The description of the theorem, Zeta functions of Picard modular surfaces (R. P. Langlands and D. Ramakrishnan, eds.), Publications CRM, Montreal, 1992, pp. 255-301. MR 93d:11055

28. Jian-Shu Li, Non-vanishing theorems for the cohomology of certain arithmetic quotients, J. Reine Angew. Math. 428 (1992), 177-217. MR 93e:11067

29. C. Moeglin, M.-F. Vigneras, and J.-L. Waldspurger, Correspondances de Howe sur un corp p-adiques, Lecture Notes in Math. 1291, Springer-Verlag, New York, 1987. MR 91f:11040

30. C. Moen, The dual pair $(U(1), U(1))$ over a p-adic field, Pacific J. Math. 158 (1993), 365-386. MR 94a:22036

31. I. I. Piatetski-Shapiro and S. Rallis, L-functions for classical groups, Lecture Notes in Math. 1254, Springer-Verlag, New York, 1987, pp. 1-52. MR 89b:11046

32. I. I. Piatetski-Shapiro and S. Rallis, $\epsilon$-factors of representations of classical groups, Proc. Symp. Nat. Acad. Sci. 83 (1986), 4589-4593. MR 88a:11053

33. I. I. Piatetski-Shapiro and S. Rallis, Rankin triple L-functions, Compositio Math. 64 (1987), 31-115. MR 89k:11037

34. D. Prasad, Trilinear forms for representations of GL(2) and local $\epsilon$-factors, Compositio Math. 75 (1990), 1-46. MR 91i:22023

35. D. Prasad, On the local Howe duality correspondence, International Math. Res. Notices 11 (1993), 279-287. MR 94k:22036

36. S. Rallis, On the Howe duality conjecture, Compositio Math. 51 (1984), 333-399. MR 85g:22034

37. S. Rallis, Langlands functoriality and the Weil representation, Amer. J. Math. 104 (1982), 469-515. MR 84c: 10025

38. S. Rallis, Injectivity properties of liftings associated to Weil representations, Compositio Math. 52 (1984), 139-169. MR 86d:11038

39. S. Rallis, Poles of standard L-functions, Proceedings of the International Congress of Mathematics, Kyoto, Springer-Verlag, New York, 1990, pp. 833-845. MR 93f:11041

40. R. Ranga Rao, On some explicit formulas in the theory of Weil representation, Pacific J. of Math. 157 (1993), 335-371. MR 94a:22037

41. J. Rogawski, Automorphic representations of unitary groups in three variables, Annals of Math. Studies 123, Princeton University Press, Princeton, N.J., 1990. MR 91k:22037 
42. J. Rogawski, The multiplicity formula for A-packets, Zeta functions of Picard modular surfaces (R. P. Langlands and D. Ramakrishnan, eds.), Publications CRM, Montreal, 1992, pp. 395419. MR 93f: 11042

43. G. Shimura, On Eisenstein series, Duke Math. J. 50 (1983), 417-476. MR 84k:11019

44. W. Sweet, A computation of the gamma matrix of a family of p-adic zeta integrals, preprint 1991.

45. J. Tate, Fourier analysis in number fields and Hecke's zeta function, Algebraic Number Theory (Cassels and Frohlich, ed.), Academic Press, 1968. MR 36:121

46. J. Tate, Number theoretic background, Automorphic Forms, Representations and $L$-Functions, part 2, Proc. Symp. Pure Math., vol. 33, AMS, Providence, 1979, pp. 3-22. MR 80m:12009

47. J. Tunnell, Local $\epsilon$-factors and characters of $G L_{2}$, Amer. J. Math. 105 (1983), 1277-1308. MR 86a:22018

48. D. Vogan, The local Langlands conjecture, Representation theory of groups and algebras, AMS, Contemporary Math. 145, Providence, R.I., 1993, pp. 305-379. MR 94e:22031

49. J.-L. Waldspurger, Sur les valeurs de certains fonctions L automorphes en leur centre de symétrie, Compositio Math. 54 (1985), 173-242. MR 87g:11061b

50. J.-L. Waldspurger, Demonstration d'une conjecture de duality de Howe dans le case p-adiques, $p \neq 2$, Festschrift in Honor of Piatetski-Shapiro, vol. 2, Israel Math. Conf. Proc., 1990, pp. 267324. MR 93h:22035

51. J.-L. Waldspurger, Correspondance de Shimura et quaternions, Forum Math. 3 (1991), 219307. MR 92g: 11054

52. Tonghai Yang, Theta liftings and L-functions of elliptic curves, Thesis, Univ. of Maryland (1995).

Department of Mathematics, Brandeis University, Waltham, Massachusetts 02254

E-mail address: harris@brandeis.bitnet

Department of Mathematics, University of Maryland, College Park, Maryland 20742

E-mail address: ssk@math.umd.edu

Department of Mathematics, Florida International University, University Park, MiAMI, FLORIDA 33199-0001

E-mail address: sweetwj@fiu.edu 